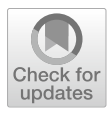

Cite as

Nano-Micro Lett.

(2021) 13:149

Received: 29 March 2021

Accepted: 19 May 2021

Published online: 23 June 2021

(c) The Author(s) 2021

\section{Nanoparticle-Cartilage Interaction: Pathology-Based Intra-articular Drug Delivery for Osteoarthritis Therapy}

\author{
Xu Li ${ }^{1,2,4}$, Bingyang Dai ${ }^{1,2,4}$, Jiaxin Guo ${ }^{1,2,4}$, Lizhen Zheng ${ }^{1,2,4}$, Quanyi Guo ${ }^{3}$, Jiang Peng ${ }^{3}$,
} Jiankun $\mathrm{Xu}^{1,2,4}$, Ling Qin ${ }^{1,2,4} \bowtie$

\title{
HIGHLIGHTS
}

- Nanoparticles hold considerable promise for controlling the local pharmacokinetics of therapeutic agents during osteoarthritis therapy.

- The advantages of nanoparticles, the bioactive design, the transports and interactions within cartilage, and therapeutic mechanisms are discussed.

- This review proposes future strategies to design more intelligent and multi-functional nanomaterials as delivery systems for cartilage therapy.

\begin{abstract}
Osteoarthritis is the most prevalent chronic and debilitating joint disease, resulting in huge medical and socioeconomic burdens. Intra-articular administration of agents is clinically used for pain management. However, the effectiveness is inapparent caused by the rapid clearance of agents. To overcome this issue, nanoparticles as delivery systems hold considerable promise for local control of the pharmacokinetics of therapeutic
\end{abstract}

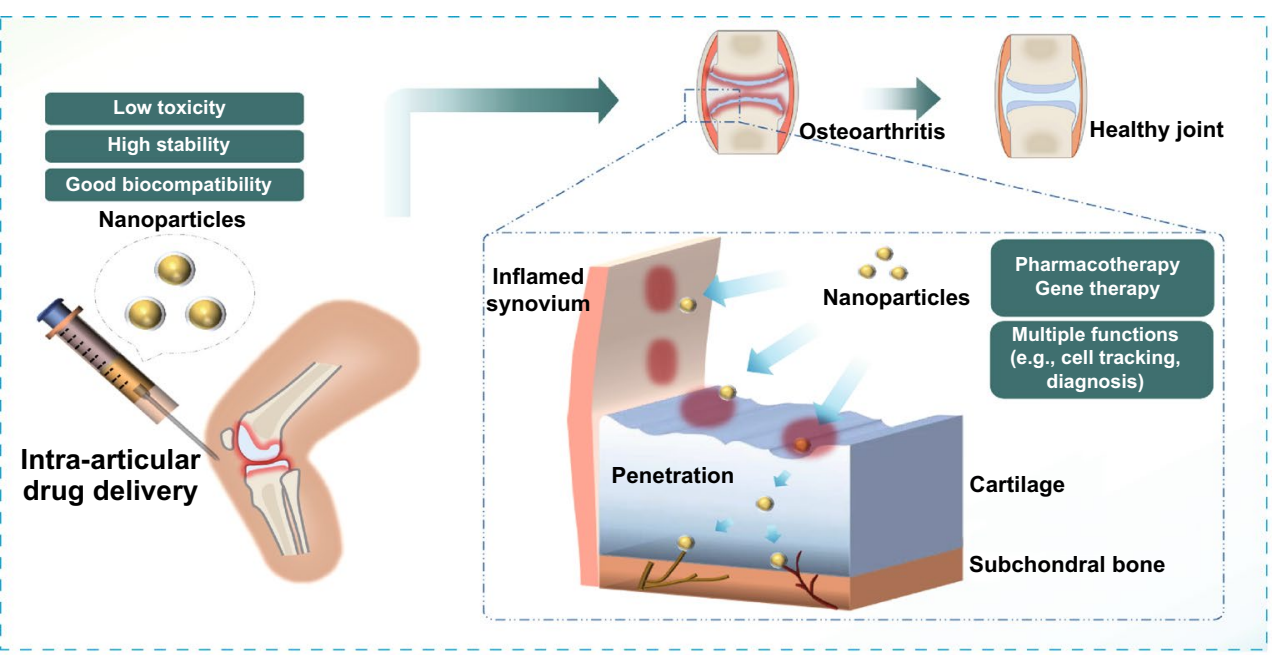
agents. Given the therapeutic programs are inseparable from pathological progress of osteoarthritis, an ideal delivery system should allow the release of therapeutic agents upon specific features of disorders. In this review, we firstly introduce the pathological features of

Jiankun Xu, jiankunxu@cuhk.edu.hk; Ling Qin, lingqin@cuhk.edu.hk

1 Musculoskeletal Research Laboratory, Department of Orthopedics and Traumatology, The Chinese University of Hong Kong, Sha Tin,

Hong Kong, SAR, People's Republic of China

2 Joint Laboratory of Chinese Academic of Science and Hong Kong for Biomaterials, The Chinese University of Hong Kong, Sha Tin, Hong Kong, SAR, People's Republic of China

3 Beijing Key Lab of Regenerative Medicine in Orthopedics, Key Laboratory of Musculoskeletal Trauma and War Injuries PLA, Institute of Orthopedics, Chinese PLA General Hospital, Beijing, People's Republic of China

4 Innovative Orthopedic Biomaterial and Drug Translational Research Laboratory, Li Ka Shing Institute of Health Sciences, The Chinese University of Hong Kong, Sha Tin, Hong Kong, SAR, People's Republic of China 
osteoarthritis and the design concept for accurate localization within cartilage for sustained drug release. Then, we review the interactions of nanoparticles with cartilage microenvironment and the rational design. Furthermore, we highlight advances in the therapeutic schemes according to the pathology signals. Finally, armed with an updated understanding of the pathological mechanisms, we place an emphasis on the development of "smart" bioresponsive and multiple modality nanoparticles on the near horizon to interact with the pathological signals. We anticipate that the exploration of nanoparticles by balancing the efficacy, safety, and complexity will lay down a solid foundation tangible for clinical translation.

KEYWORDS Nanoparticle; Drug delivery; Osteoarthritis; Articular cartilage; Nanomedicine

\section{Introduction}

Osteoarthritis (OA) is the most prevalent chronic and debilitating joint disease and a leading cause of disability of elderly individual due to daily wear and tear of cartilage. Chronic pain, joint instability, stiffness, and radiographic joint space narrowing are major clinical symptoms, affecting about $10 \%$ of men and $18 \%$ of women over 60 years of age $[1,2]$. Consequently, the resultant individual and socioeconomic burdens are huge $[1,2]$.

As aneural and avascular tissue, articular cartilage is of weak regeneration ability. Once damaged, it is hardly to be repaired and inescapable to degenerate. Because of the rapid clearance of synovial fluid and the barrier of dense natural cartilage extracellular matrix (ECM), the effectiveness of traditional intra-articular therapies, analgesics, glucocorticoids, and hyaluronic acid is still far from satisfactory [3]. To overcome these issues, nanoparticle is a desirable delivery system with the most suitable size to penetrate past the superficial zone of the cartilage and locally control the pharmacokinetics of therapeutic agents. As soon as nanoparticles are delivered into articular cavity, nano-cartilage interactions occur throughout their transport and penetration within the matrix. Apart from pain relief, nanoparticles-based therapy is also promising to attenuate cartilage degeneration and even promote regeneration. Armed with an updated understanding of the pathological features and OA pain, we may develop innovative nanoparticles for targeting multiple tissues, such as cartilage, nerves and/or blood vessels in synovium and subchondral bone, to enhance the therapeutic efficacy.

Herein, we review the pathological features of OA, limitations of current intra-articular therapies, and the advantages of nanoparticles for sustained drug delivery. Then, we summarize how to take advantages of these unique nanoscale properties, components, size, and surface chemistry, to facilitate their transports and interactions within cartilage. Furthermore, we highlight advances in the therapeutic mechanisms of nanoparticles. Finally, we place an emphasis on the design of the anticipated "smart" bioresponsive and multi-functional nanoparticles as the next-generation delivery system to interact with the pathological abnormalities and at the same time achieve controlled release. We anticipate that the exploration of nanoparticles by balancing the efficacy, safety, and complexity will lay down a foundation for clinical translations.

\section{Limitations of Current OA Therapy Demands Research and Development (R\&D) of Effective Drug Delivery Systems}

\subsection{Pathological Mechanisms of OA}

The primary function of articular cartilage is to bear loading during motion. Articular cartilage is hyaline cartilage in nature with very limited support of blood vessels, nerves, or lymphatics. Articular cartilage mainly consists of highly specialized chondrocytes encapsulated in ECM. The slow turnover of ECM in cartilage makes the regeneration difficult in skeletally mature individuals. For example, it takes up to 25 years for the turnover of proteoglycans and the halflife of type II collagen is between 100 and 400 years [4-7].

Chondrocytes, which are sensitive to the changes of chemical and mechanical environment in OA, increase synthetic activity to generate collagen type $\mathrm{X}$ (matrix degradation associated products) at early stage of the disease [8]. Chondroptosis (apoptosis of chondrocytes) is increased in OA caused by oxidative and nitrosative stress, inflammatory cytokines, and mechanical stress [8]. At the same time, several inflammatory cytokines are produced, including interleukin (IL) $1 \beta$, IL 6, and tumor necrosis factor (TNF) $\alpha$, and matrix-degrading enzymes (the matrix metalloproteinases (MMP) and a disintegrin and metalloproteinase with thrombospondin-like motifs (ADAMTS)) [8]. Ultimately, these enzymes mediate 
the degradation of cartilage ECM [8]. These catabolic factors also activate a series of pathways such as nuclear factor kappa B (NF-kB) and Wnt signaling, which play important roles during the pathological progress of OA [9-11]. At a later stage, inflammatory fluids filling the joint capsule cause swelling, more pain, and stiffness [8]. Cartilage becomes hypocellular with impaired metabolic flexibility [6].

There is an imbalance between catabolic and anabolic metabolism of articular cartilage in OA (Fig. 1) [12].
Although the synthesis of ECM increases, it is no longer able to fully compensate cartilage degradation [12]. MMP13 is mainly in charge of degrading collagen and ADAMTS4 and 5 is for the destruction of aggrecan [13]. Decrease in the concentration of glycosaminoglycans (GAGs) and the disruption of the collagen orientation are therefore presented during OA. Consequently, the permeability of cartilage and interstitial fluid velocities within the matrix are increased [14]. Initially, changes of integrity at the surface disrupt
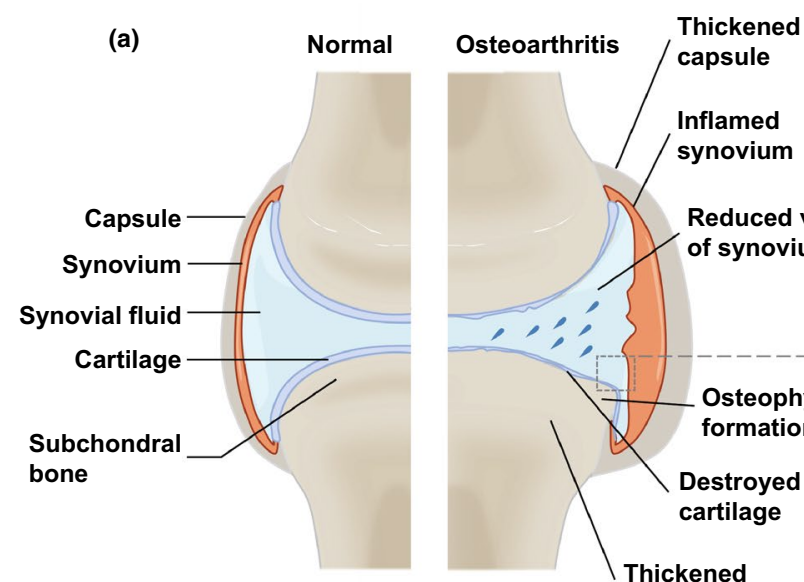

\section{(1)}
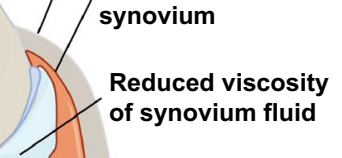

(b)
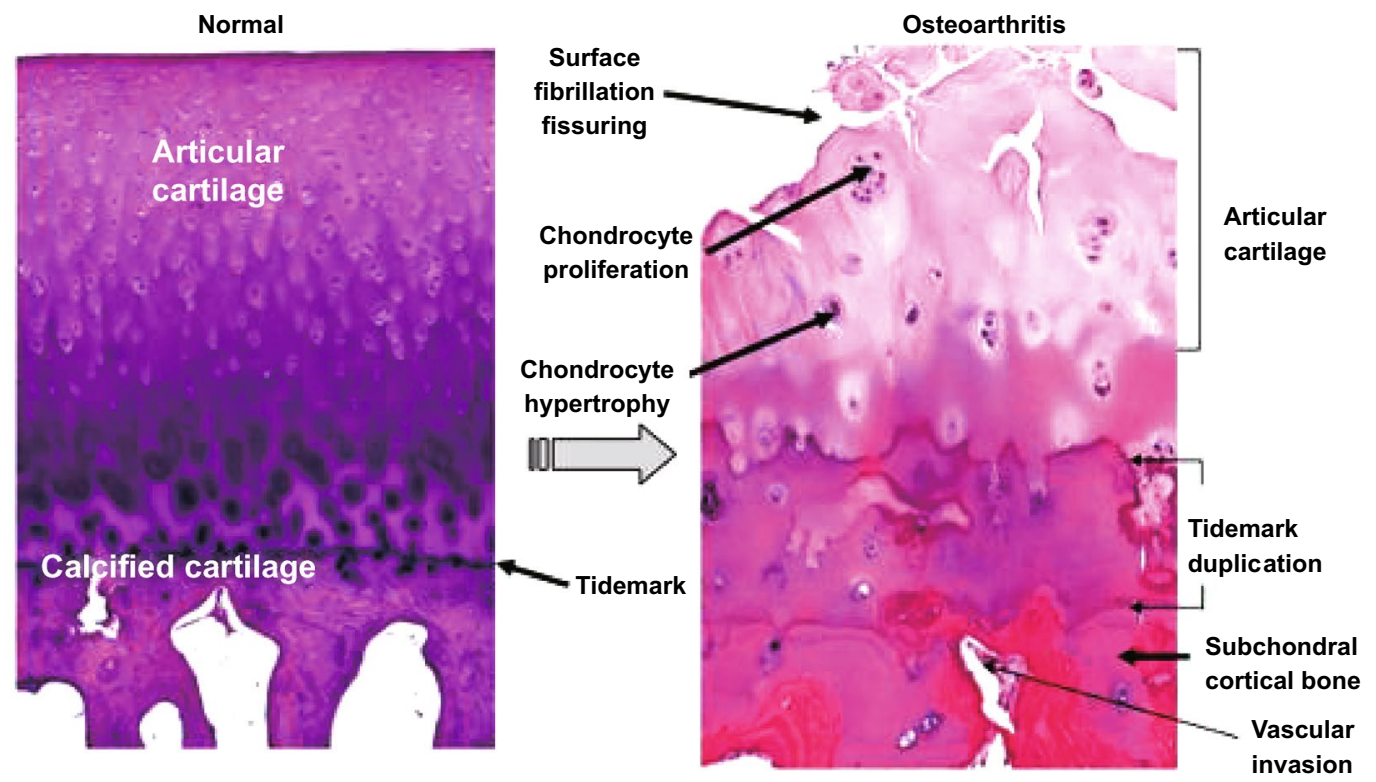

Fig. 1 Pathological changes of OA. a Drawing of structural changes and signaling pathways of OA. b Histologic cross section of normal cartilage (left picture) and cartilage affected by end-stage OA (right picture). End-stage OA is characterized by articular cartilage injury, chondrocyte proliferation and hypertrophy, tidemark duplication, subchondral bone thickening, and vascular invasion. Reproduced with permission [277]. Copyright 2016, Elsevier Inc. Abbreviations: IL-1 $\beta$, interleukin 1 $\beta$; IL-6, interleukin 6; ADAMTS-4, a disintegrin and metalloproteinase with thrombospondin-like motifs 4; ADAMTS-6, a disintegrin and metalloproteinase with thrombospondin-like motifs 6; MMP-1, matrix metalloproteinases-1; MMP-13, matrix metalloproteinases-13; OA, osteoarthritis 
cartilage composition and increase the susceptibility to physical forces $[8,15]$. Without proper therapy, fissures will form in deep cartilage along with calcified cartilage zone expansion [16]. Additionally, the degradation of cartilage leads to the remodeling of subchondral bone and subchondral thickening $[8,15]$. This process resembles chondrocyte differentiation during embryogenesis accompanied by the formation of osteophytes and subchondral cysts $[8,15]$. Synovitis is another key feature of OA. Notable tissue hypertrophy, synoviocytes proliferation and increased vascularity contribute to the release of inflammatory factors [15]. Additionally, persistent joint inflammation leads to lymph node collapse and reduced lymphatic drainage, which contribute to severe synovitis and joint erosion [17].

\subsection{Inadequate Clinical Therapy Efficacy}

Pain medication is the mainstay of pharmacotherapy for OA $[18,19]$. Oral administration of nonsteroidal anti-inflammatory drugs (NSAIDs), cyclooxygenase-2 (COX-2) inhibitors, and acetaminophen has shown to soothe the pain and improve function [18-21]. However, significant adverse events and low local concentration of drugs are the main obstacles limiting their clinical applications. For example, oral administration of NSAIDs increases the risk of heart attack and induces kidney or gastrointestinal disorders [22].

Intra-articular drug delivery has various advantages over the systemic administration, such as increased local bioavailability throughout the joint capsule and fewer adverse events [3]. Intra-articular delivery of corticosteroids, hyaluronic acid, platelet-rich plasma and glucosamine or chondroitin can increase local bioavailability and serve as another strategy to reduce pain and relieve symptoms [3, 18-21, 23]. However, synovial fluid with complex biological composition and high viscosity affects drug's properties and diffusion. The low residence time of drugs during articular cavity administration is mainly caused by the rapidly cleared from the synovial fluid [3]. The dense networks of collagen fibers and proteoglycans of cartilage ECM are also obstacles for drug absorption. Furthermore, cartilage ECM is avascular and densely packed with negative charged molecules, which particularly makes the diffusion of the negative charged drug even more difficult. Consequently, the half time of NSAIDs and soluble corticosteroids injected into the joint capsule is about $1-4 \mathrm{~h}$, whereas hyaluronic acid can be cleared within
$26 \mathrm{~h}[24,25]$. Repeated injections as the simplest way to increase therapeutic efficacy bring other problems such as increased risk of infection, joint disability, and the resultant high cost. Limitations of current pharmacological therapy for OA are concluded in Table 1. With the degeneration of cartilage, these therapies become less efficient, and the ultimate choice is joint replacement. Thus, drug delivery system of higher efficacy is required to overcome these obstacles.

\subsection{Advantages of Nanoparticles for the Treatment of Cartilage Disease}

Nanoparticles refer to submicron particles with the dimension from 1 to $100 \mathrm{~nm}$, which is about one thousand times smaller than chondrocytes (Fig. 2a). The controllable size endows nanoparticles the feasibility of direct intra-articular injections. Nanoparticles as carriers can incorporate drugs in the surface or matrix to protect drugs from enzymatic degradation, improve their penetrations across cartilage matrix, and modulate drug pharmacokinetics, which is beneficial for balancing the efficacy and the toxicity of therapeutic compounds (Fig. 2b). In order to optimize the degradation, toxicity, and mechanical properties, hybrid nanoparticles combining two or more components may have superior properties than singlecomponent materials. By adjusting physicochemical properties or decorating with moieties, nanoparticles can be functionalized to target components and/or cells, e.g., chondrocytes, in the cartilage. Biocompatible and biodegradable materials such as polymers or solid lipids make up nanoparticles to enable controlled and sustained drug release. The increased specific surface area and surface to volume ratio are also beneficial for the dissolution and release of drugs [26]. Moreover, modifications can be performed by grafting other functional units for imaging. Other technological advantages includes high stability (e.g., long shelf life), feasibility of incorporation of both hydrophilic and hydrophobic substances and feasibility of variable routes of administration (including intra-articular injection or in combination with scaffold or hydrogel) [27].

Nanoparticle-based local gene transfer can alter the expression of the endogenous genes to prevent or slow the pathological progress of OA by introducing genes, such as DNA, mRNA, siRNA, and miRNA, into the target cells. Compared with the naked genetic molecules, nanoparticles hold potential to provide safe, efficient, and controllable gene delivery by manipulating properties 


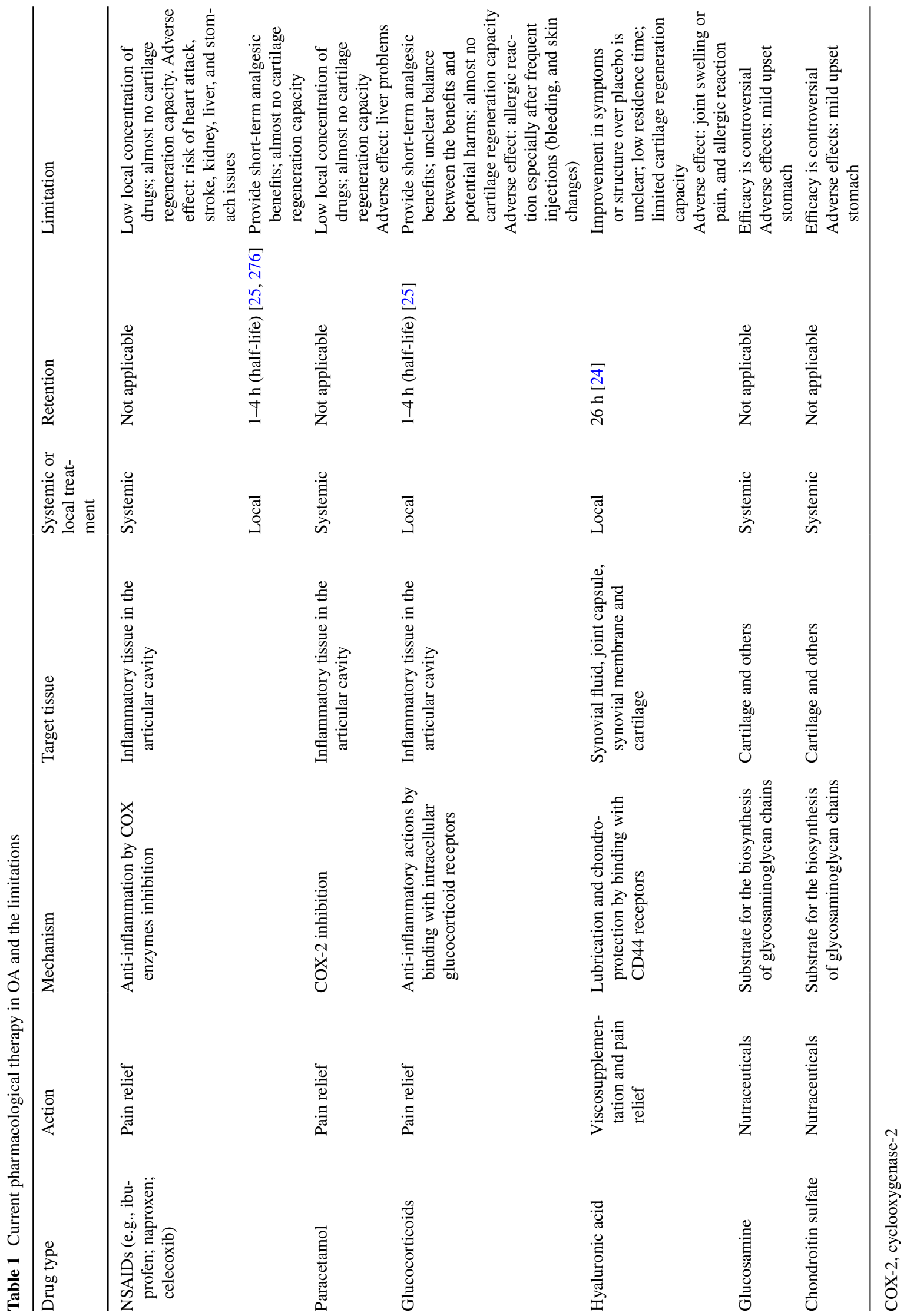


(a)

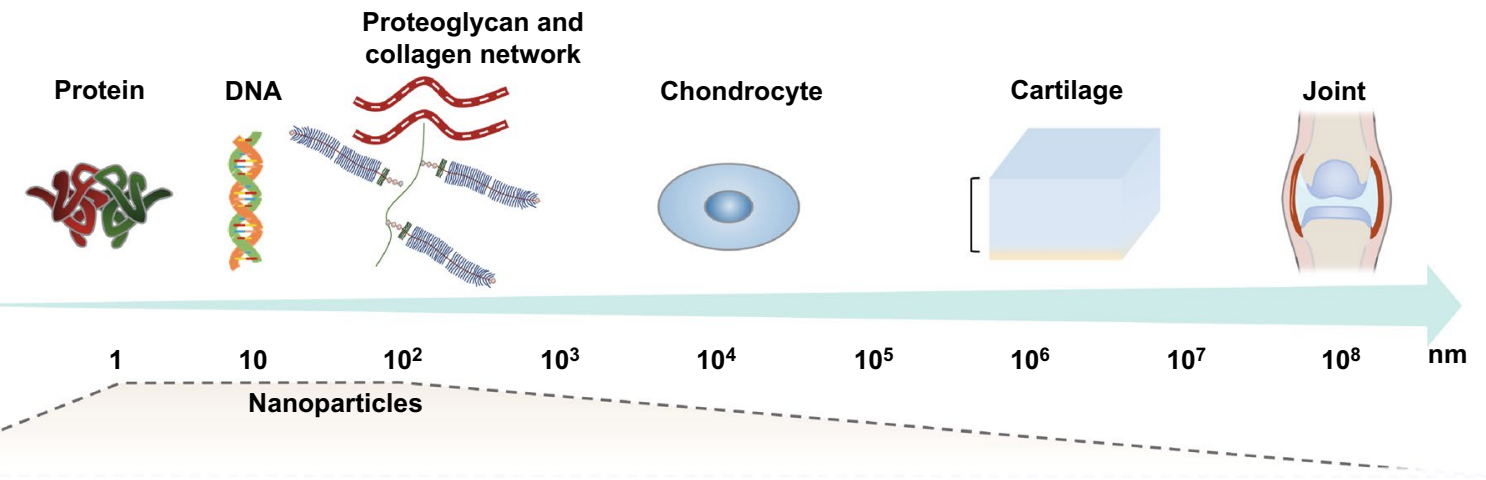

(b)

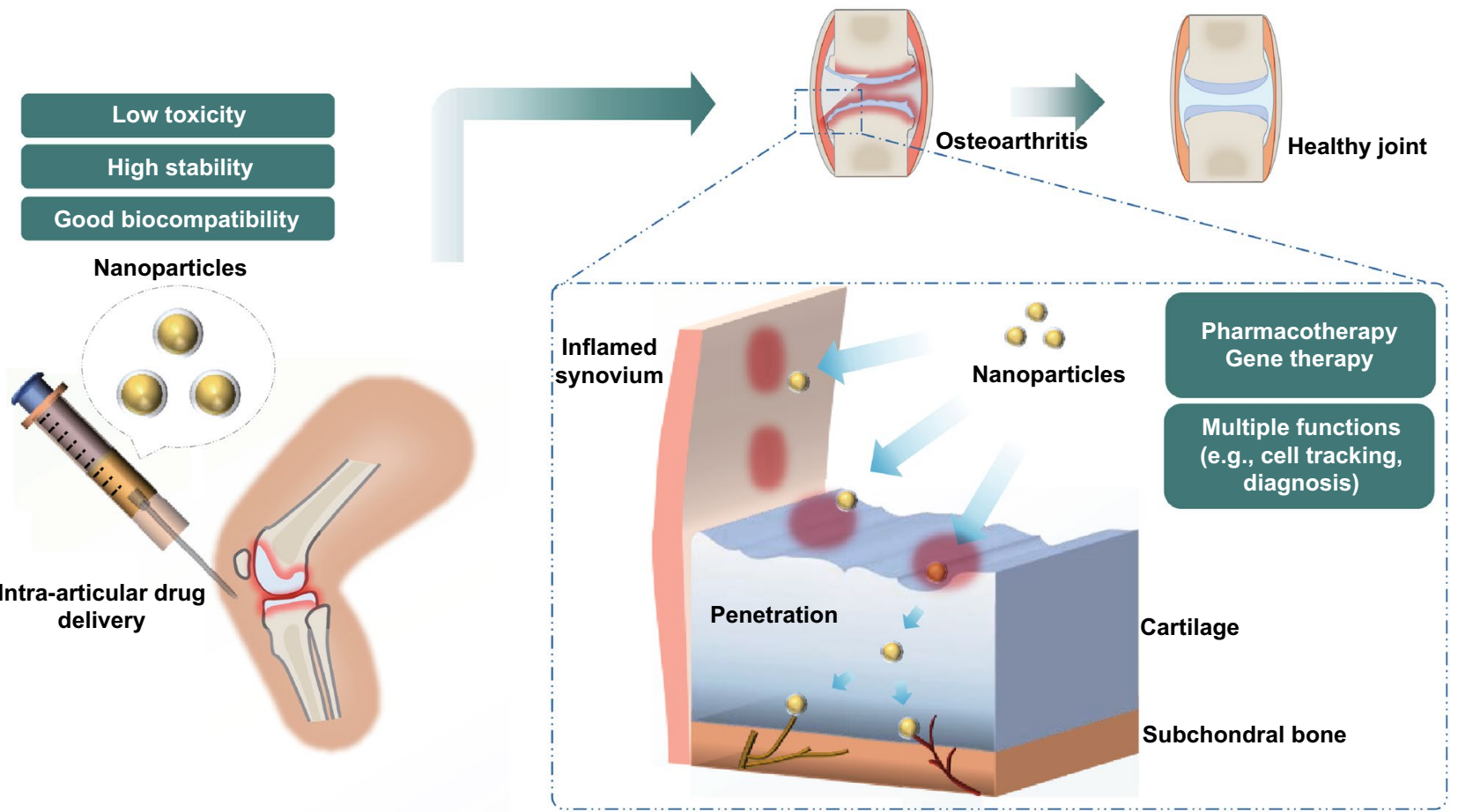

Fig. 2 Properties and application schemas of nanoparticles for the treatment of cartilage disease. a Size of nanoparticles compared with different components in joint. b Application schemas of nanoparticles for intra-articular delivery

such as encapsulation efficiency, stability, degradation, endocytosis, endosomal escape, and toxicity.

\section{Transports of Nanoparticles Within the Joint Cavity}

\subsection{Pharmacokinetics and Biodistribution of Nanoparticles}

The proper space-time retention in the joint cavity is the prerequisite for ensuring nanoparticles' effective interaction with different components in the joint. Therefore, real-time monitoring of pharmacokinetics and biodistribution (PK/BD) of nanoparticles is important to define their therapeutic effect. Nanoparticle-based intra-articular delivery systems reduce the distribution to the reticuloendothelial organs and increase drugs' half-lives by at least tenfold than free drugs [28, 29]. Pharmacokinetics of nanoparticles within cartilage depends on their chemical and physical properties, including size, charge, and surface chemistry, as well as the pathological state of joint cavity. Nanoparticles or their encapsulated drugs exit joints via 
the small blood vessels and lymphatic system (Fig. 3a) [30, 31]. The small blood vessels are the main channels for the clearance of small particles $[30,31]$. In contrast, lymphatic pathway eliminates particles or their degradation products independently of their size [30, 31]. A study using mouse model proves that particles are preferentially drained through the iliac lymphatic nodes near the aortic bifurcation, and the remaining goes through hind leg lymphatic drainage to enter the inguinal lymphatic node [31].

Theoretically, the retention of drug carriers in cartilage depends on the net flux penetrating cartilage from synovial fluid and their efflux rate from the lymphatics and subsynovial capillaries. Therefore, the net influx penetrating cartilage from synovial fluid must reach therapeutic levels before the clearance to ensure effective drug delivery. If the concentration of nanoparticles within articular cavity reaches a high level immediately after the delivery. If the concentration of nanoparticles in synovial fluid is higher than that in the cartilage, the nanoparticles will penetrate into the cartilage matrix. When the concentration in synovial fluid decreases with the clearance of lymphatic and blood vessels, nanoparticles diffuse outward from cartilage into synovial fluid. Detailed pharmacokinetics of drug delivery systems has been well described in previous literature [32].

Synovial inflammation in OA increases capillary permeability and alters the lymphatic permeability (displayed initial compensatory expanding phase followed by a collapsed phase) [17]. Synovial fluid and serum tend to equilibrate through synovium intercellular gaps in a normal status; in OA, synovial inflammation-associated increased capillary permeability facilitates the elimination of nanoparticles from joint [31]. However, the clearance of nanoparticles in OA joint is impaired compared with the healthy joint, which may be associated with synovial thickening [33, 34]. Ageing associated metabolic changes, including less range of joint motion and decreased lubricating fluid, may also impede the clearance of nanoparticles. In a rat OA model, increased particle retention (in 14 days) has been found in 15-month rat knees, compared to 5- and 10-month rat knees (Fig. 3c) [33]. Although no animal study precisely recapitulates how the pathophysiology of OA affects the clearance of nanoparticles from lymphatic pathway, we can speculate that the initial 'expansion' phase of lymphatic vessels during moderate experimental arthritis may facilitate efficient lymphatic clearance; in contrast, the collapsed phase of lymphatic vessel characterized by lymphatic vessel structural damage and loss of contraction may reduce lymphatic clearance of nanoparticle or their encapsulated drugs [17].

\subsection{Size-Dependent Penetration Within Cartilage Matrix}

The dense ECM and avascularity structure determine the transports and interactions of nanoparticles within cartilage. Human knee articular cartilage is about 2 to $4 \mathrm{~mm}$ thick, which mainly consists of three zones-the superficial (tangential) zone ( $10 \%$ to $20 \%)$, the middle (transitional) zone ( $\sim 40 \%$ to $60 \%$ ), and the deep zone ( 30 to $40 \%$ ) (Fig. 3b) $[32,35]$. Superficial zone contains a high number of flattened chondrocytes and collagen fibers aligned tightly parallel to the articular surface. The mesh size of collagen type II fibrillar network in the superficial zone is about $50-60 \mathrm{~nm}$ $[36,37]$. The space between the side chains of the proteoglycan network is about $20 \mathrm{~nm}[37,38]$. This zone with the good tensile properties is responsible for resisting the sheer, tensile, and compressive forces. The middle (transitional) zone is responsible for the resistance to compressive forces that contains proteoglycans and thicker obliquely organized collagen fibrils $[32,35]$. The spherical chondrocytes are distributed at low density [32, 35]. In deep zone, chondrocytes are parallel to the collagen fibers and columnar to the joint line $[32,35]$. Proteoglycan content is the highest, and the resistance to compressive forces is the greatest $[32,35]$. The calcified zone containing scarce and hypertrophic chondrocytes distinguishes the deep zone from the subchondral bone $[32,35]$.

The depth at which the nanoparticles can diffuse depends on both the pores in the cartilage nano/microstructure and the size of the particles. The increased network density of proteoglycan with the thickness of cartilage makes the penetration of nanoparticles more difficult. Whereas larger sized nanoparticles do not penetrate into cartilage, smaller ones are capable of penetrating deeper (Fig. 3f). As the pore size of collagen type II fibrillar network is about $50-60 \mathrm{~nm}$ in the superficial zone $[36,37]$, nanoparticles beyond this size may not be able to enter the cartilage matrix efficiently. Human-thickness bovine cartilage is commonly employed to investigate the penetration of nanoparticles. The accumulation of 38-nm nanoparticles of poly(propylene sulfide) (PPS) in bovine cartilage cartilage matrix is 14.9 -fold higher than $96 \mathrm{~nm}$ nanoparticles 


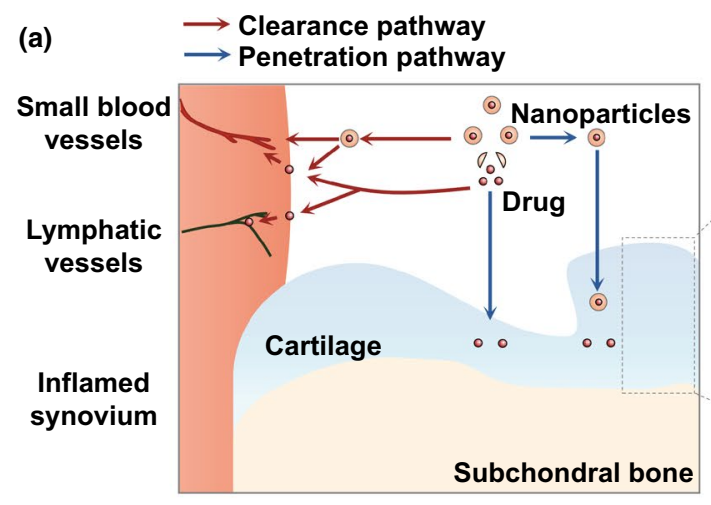

(b)

(c)

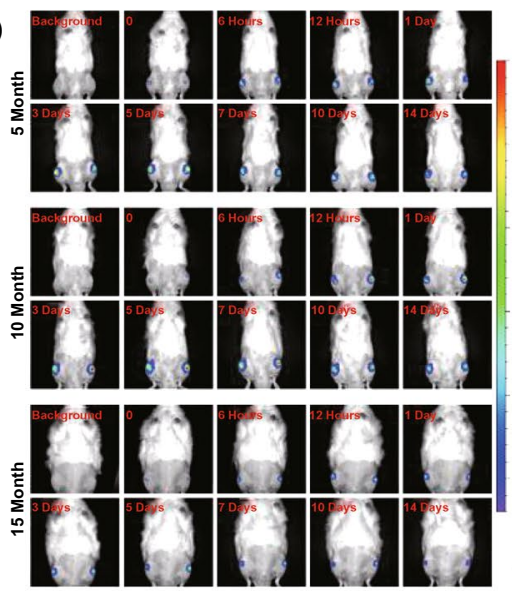

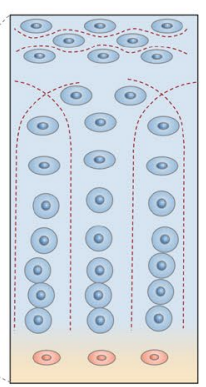

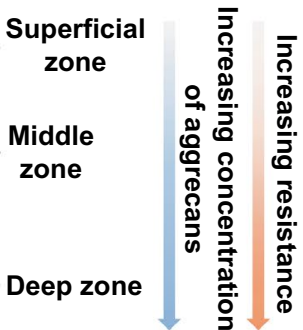

Calcified zone

(d) TGa-NP TGFa-NP

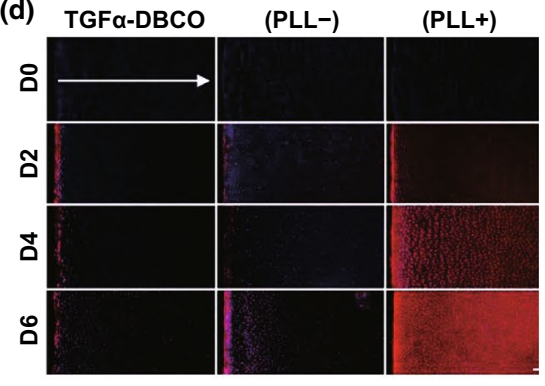

$2.0 \rightarrow 1.2 \rightarrow$ TGFa-DBCO

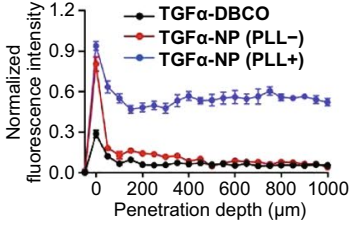

(e) Osteochnodral

1.0

Radiant Efficiency $\left(\frac{\mathrm{p} / \mathrm{sec} / \mathrm{cm}^{2} / \mathrm{sr}}{\mu \mathrm{W} / \mathrm{cm}^{2}}\right.$ Color Scale
Min $=6.00 \mathrm{e}$ $\operatorname{Min}=6.00 \mathrm{e} 7$
$\operatorname{Max}=6.00 \mathrm{e} 8$ Penetration depth $(\mu \mathrm{m})$

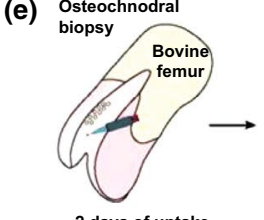

2 days of uptake
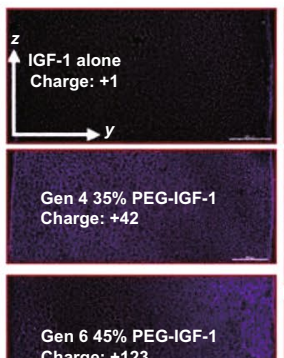

Charge: +123 (f)

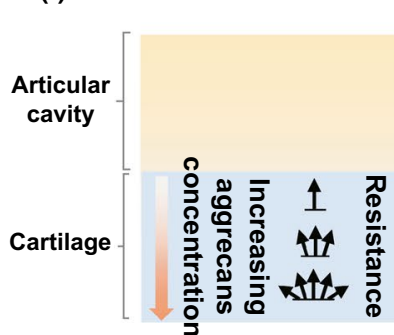

Isolate cartilage explant

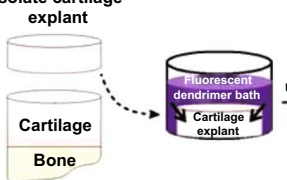

6 days of uptake
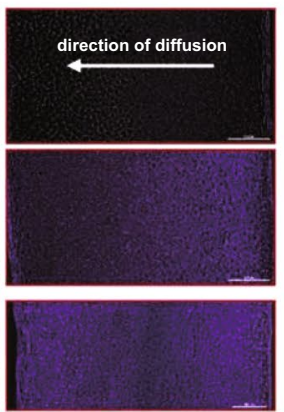

Cartilage penetration assay and processing

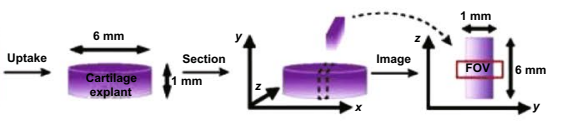

2 days of uptake

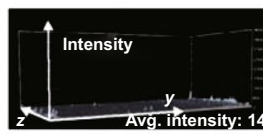

6 days of uptake
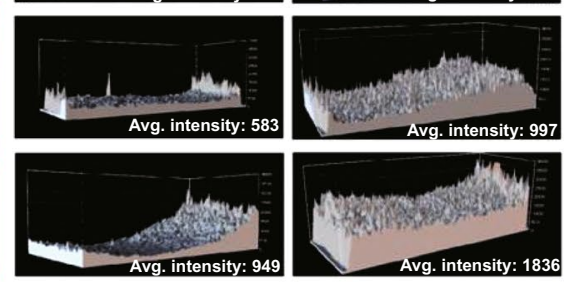

Surface-modified

Nanoparticles Nanoparticles Nanoparticles large sized Increased penetration of $(>60 \mathrm{~nm}) \quad(15 \sim 60 \mathrm{~nm})$ nanoparticles nanoparticles in OA cartilage

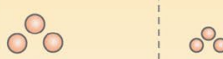

00

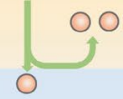

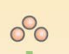

$\mathrm{O}^{\circ}$
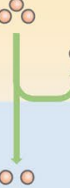

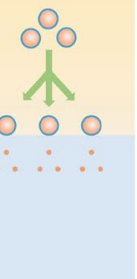

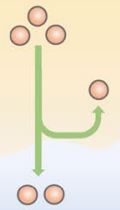

00
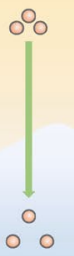
4Fig. 3 Interaction of nanoparticles with cartilage. a Clearance and biodistribution of nanoparticles within joint cavity. $\mathbf{b}$ Cartilage layers as barriers of drug penetration. c Retention of nanoparticles in OA and the contralateral joints in rats with different ages. Reproduced with permission [33]. Copyright (C) 2020 Elsevier B.V. d Penetration of $25.93 \mathrm{~nm}$ nanoparticles within bovine articular cartilage with similar joint cartilage thickness to human. Reproduced with permission [34]. Copyright (C) 2021 American Association for the Advancement of Science. e Penetration of $25.93 \mathrm{~nm}$ nanoparticles within bovine articular cartilage [40]. Reproduced with permission Copyright (C) 2018 American Association for the Advancement of Science. f Penetration of different sizes of nanoparticles into the cartilage matrix. Penetration depths of nanoparticles within cartilage matrix is in a size-dependent manner. Surface-modified nanoparticles with large size can be functionalized binding to the cartilage surface for drug release. Penetration of nanoparticles increases in OA cartilage compared with healthy cartilage Copyright (C) 2020 Elsevier B.V. d Penetration of $25.93 \mathrm{~nm}$ nanoparticles within bovine articular cartilage with similar joint cartilage thickness to human. Reproduced with permission [34]. Copyright (C) 2021 American Association for the Advancement of Science. e Penetration of $25.93 \mathrm{~nm}$ nanoparticles within bovine articular cartilage [40]. Reproduced with permission Copyright (C) 2018 American Association for the Advancement of Science. f Penetration of different sizes of nanoparticles into the cartilage matrix. Penetration depths of nanoparticles within cartilage matrix is in a size-dependent manner. Surface-modified nanoparticles with large size can be functionalized binding to the cartilage surface for drug release. Penetration of nanoparticles increases in OA cartilage compared with healthy cartilage

after intra-articular delivery for $24 \mathrm{~h}$, although most of the nanoparticles are still withheld in the cartilage surface [37]. Poly[L-lysine-block-poly( $\varepsilon$-caprolactone)] (PLL)-polycaprolactone (PCL) nanoparticles $(25.93 \mathrm{~nm})$ can efficiently bound to the surface of bovine cartilage explants with articular cartilage thickness similar to that of human at day 2 and gradually penetrated inside by at least $1 \mathrm{~mm}$ by day 6 (Fig. 3d) [34]. Given the space between the side chains of the proteoglycan network is about $20 \mathrm{~nm}$, small $(\leq 15 \mathrm{~nm})$ nanoparticles can easily enter the cartilage matrix by binding and penetrating anionic cartilage tissue $[37,38]$. For example, a study proves that $15-\mathrm{nm}$ micelles are better than 138-nm-diameter liposomes in penetrating bovine articular cartilage [39]. At 4-h postnanoparticles treatment, micelles have already penetrated across the superficial and middle zones of bovine articular cartilage [39]. Similarly, amine terminal polyamidoamine (PAMAM) dendrimers functionalized with variable molar ratios of poly(ethylene glycol) (PEG) (diameter $<10 \mathrm{~nm}$ ) exhibit full penetration of human-thickness bovine cartilage with a $70 \%$ absorption rate (Fig. 3e) [40]. Such nanoparticles as a drug delivery system increase the residence time of insulin-like growth factor 1 (IGF-1) by tenfold for up to 30 days [40]. Another study also proves that nanoparticles diameters $\sim 5 \mathrm{~nm}$ are capable of penetrating throughout the full thickness $(1 \mathrm{~mm})$ of the normal bovine cartilage within $24 \mathrm{~h}$, while an obvious penetration gradient is also found with the treatment of particles diameters $\sim 10 \mathrm{~nm}$ [41]. Even so, this does not mean that the smaller the diameter of the nanoparticles, the better therapy efficacy. Small-size nanoparticles may be easily cleared from the joint via sub-synovial capillaries and lymphatics more rapidly. In contrast, larger-sized nanoparticles with the advantage to deliver a more sustained payload are not necessarily unsuitable for drug delivery. Therefore, there are plenty of rooms for sorting out the optimal size of nanoparticles to maximize the efficacy. Alternatively, large-sized nanoparticles floating in the articular cavity need to release sufficient drug to enable the drug's penetration within cartilage. If nanoparticles can be functionalized binding to the cartilage surface by modifying surface-functional properties, their released drugs may be able to diffuse to deeper layer of cartilage by minimizing the clearance effects (Fig. 3f).

Although rodent models are widely used to investigate the OA treatment and its underlying mechanism, large animal with thicker cartilage is more suitable for exploring the transport kinetics of nanoparticles. Therefore, bovine cartilage is the most widely used for studying the penetration of nanoparticles in the past [39-41]. The thickness of cartilage negatively affects the effective diffusion of nanoparticles [32]. Giving that the thickness of cartilage increases with animal size, nanoparticles are more likely to be cleared in large animal's cartilage [14, 32, 42, 43]. Similarly, the outward diffusion of nanoparticles is also proportional to the square of the cartilage thickness [32]. Once the concentration of nanoparticles reaches therapeutic levels, the theoretical retention time also increases with cartilage thickness $[14,32,42,43]$. Of note, the decrease in proteoglycan and collagen in OA usually increases the pore size and affects the diffusion of drugs (Fig. 3f) [14]. Nanoparticles can penetrate deeper into the proteoglycan-depleted cartilage than normal cartilage [41]. Large molecules exhibit higher diffusivities benefiting the most from the increased pore size [14]. 


\subsection{Targeting Therapy to Facilitate Nanoparticle- Cartilage Interaction}

Desirable nanoparticles should be able to functionally target to specific components and/or cells of the cartilage. The strategies can be divided into passive targeting and active targeting (Fig. 4a, b and Table 2). Passive targeting is established by improving the physicochemical properties such as size, charge, surface chemistry for preventing unspecific interactions, which needs to fully consider the unique characteristics of cartilage ECM. Negative charged cartilage offers the unique opportunity to utilize electrostatic interactions with the positive charged nanoparticles to achieve passive targeting. Accelerated augment transport, uptake and intra-tissue binding of the positive charged nanoparticles shorten the time of intra-cartilage drug to reach therapeutic concentration and extend the half-life in vivo (Fig. 4a). For example, cationic globular proteins and dendrimers can target to the anionic cartilage matrix via electrostatic attraction [34, 40, 44]. With both approaches, electrostatic interactions between positively charged nanoparticles and the negative fixed charge of cartilage ECM can be optimized to augment the transport, uptake and intra-tissue binding of such nanoparticles. For example, cationic surfactants such as didodecyldimethylammonium bromide (DMAB) can help nanoparticles achieve passive targeting to improve their retentions in cartilage [45]. The retention of DMAB PLGA nanoparticles is fourfold higher than the corresponding negatively charged nanoparticles with the presence of synovial fluid [45]. Another approach is to use the cationic domain of a therapeutic drug, such as cytokines, to enable the binding to cartilage. For example, FGF family with a cationic heparin-binding domain binds heparan sulfate GAG chain in cartilage $[46,47]$. The positively charged amino acids in the heparin-binding (HB) domain can bind to the negatively charged sulfate and carboxyl groups in heparin. In addition, heparin-binding domains can also be used to attach to other cytokines such as IGF-1 to accelerate the transport inside cartilage. For example, HB-IGF-1 fabricated by binding the heparin-binding domain of HB-EGF to the amino-terminus of IGF-1 maintains the transduction of IGF signaling through the IGF receptor and displays prolonged therapeutic effect in OA model [48-50]. Besides the physicochemical properties of nanoparticles, synovial fluid and the disease state of the cartilage also affect their retention in cartilage. For example, the retention of cationic DMAB
PLGA nanoparticles decreases by $50 \%$ in the presence of synovial fluid compared with saline [45]. The possible reason is that hyaluronic acid as an anionic, non-sulfated glycosaminoglycan in synovial fluid may influence the passive targeting of positively charged nanoparticles. More importantly, the disease state of OA negatively affects passive targeting therefore compromises the ability of the positively charged nanoparticles to penetrate the matrix. For example, the retention of cationic DMAB PLGA nanoparticles displays a threefold reduction in OA cartilage compared with healthy cartilage [45].

Active targeting is established by using conjugation chemistries to attach affinity ligands to the surface of the nanoparticles (Fig. 4a, b). Targeted cell can recognize decorated nanoparticles through ligand-receptor interactions. For example, nanoparticles decorated with collagen type II antibodies can specifically bind to cartilage and facilitate drug release inside the cells [40, 51]. In addition, several types of peptides termed the collagen hybridizing peptide/ collagentargeting peptide have been developed as moiety of nanoparticles to specifically bind to denatured collagen strands by re-forming a triple-helical structure in a fashion [52-54]. ECM (including collagen and proteoglycan) surrounding the chondrocytes has higher turnover rate [55, 56]. Chondrocytes targeting therapy can be a suitable strategy to assemble collagen and proteoglycan distribution. CD44 is expressed by chondrocytes which can be used for active targeting [57-59]. Nanoparticles covered with hyaluronic acid can specifically binds to CD44, provoking the internalization [57-59].

The extent of cartilage accumulation and joint biodistribution for the two types of targeting-system is differently affected by disease states [54]. Accumulation of active nanoparticles is increased in OA cartilage compared with healthy cartilage, indicating that active targeting strategies may be advantageous for drug delivery to diseased cartilage (Fig. 4c) [54]. However, from a translational aspect, passive targeting strategies requires fewer modifications, making production easier and more controllable, therefore reducing the cost and facilitating the translational.

\subsection{Interactions with Targeted Cells}

Penetrations of nanoparticles within cartilage result in either direct contact through cell uptake, or indirect interaction through release of nanoparticle-containing materials with 
(a)

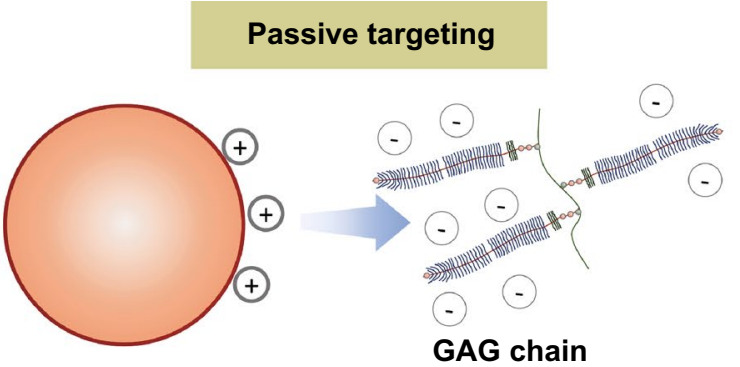

GAG chain

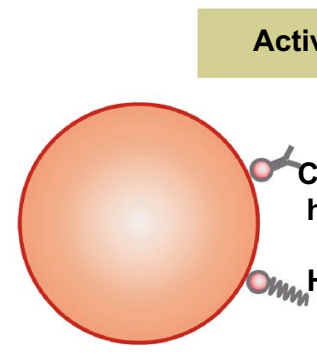

Active targeting

Collagen II antibody/ hybridizing peptide

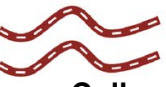

Collagen

Hyaluronic acid

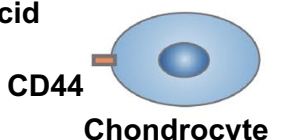

(b)

Normal states

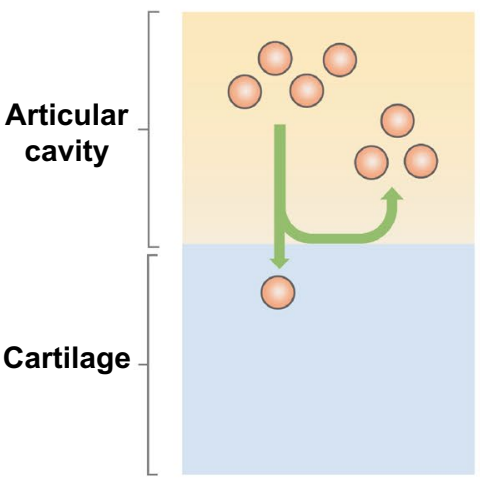

(c)
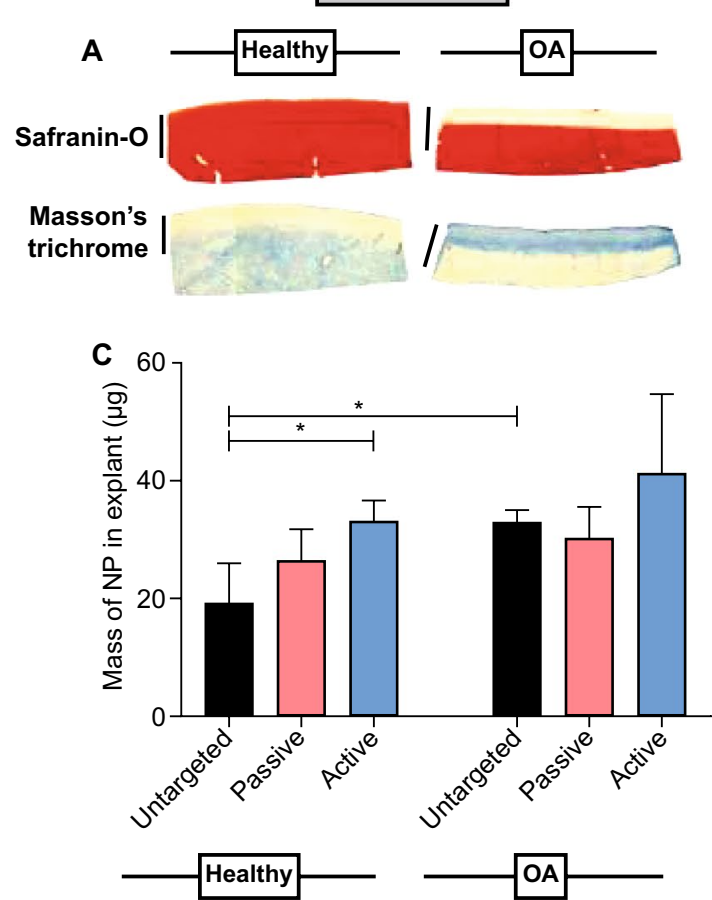

Passive targeting (i.e. cationic nanoparticles)

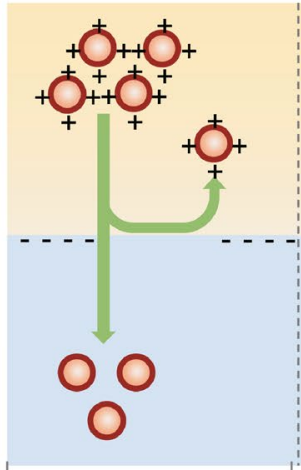

Health cartilage

Loading

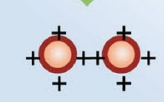

OA cartilage

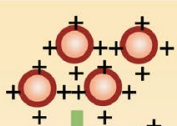

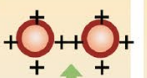
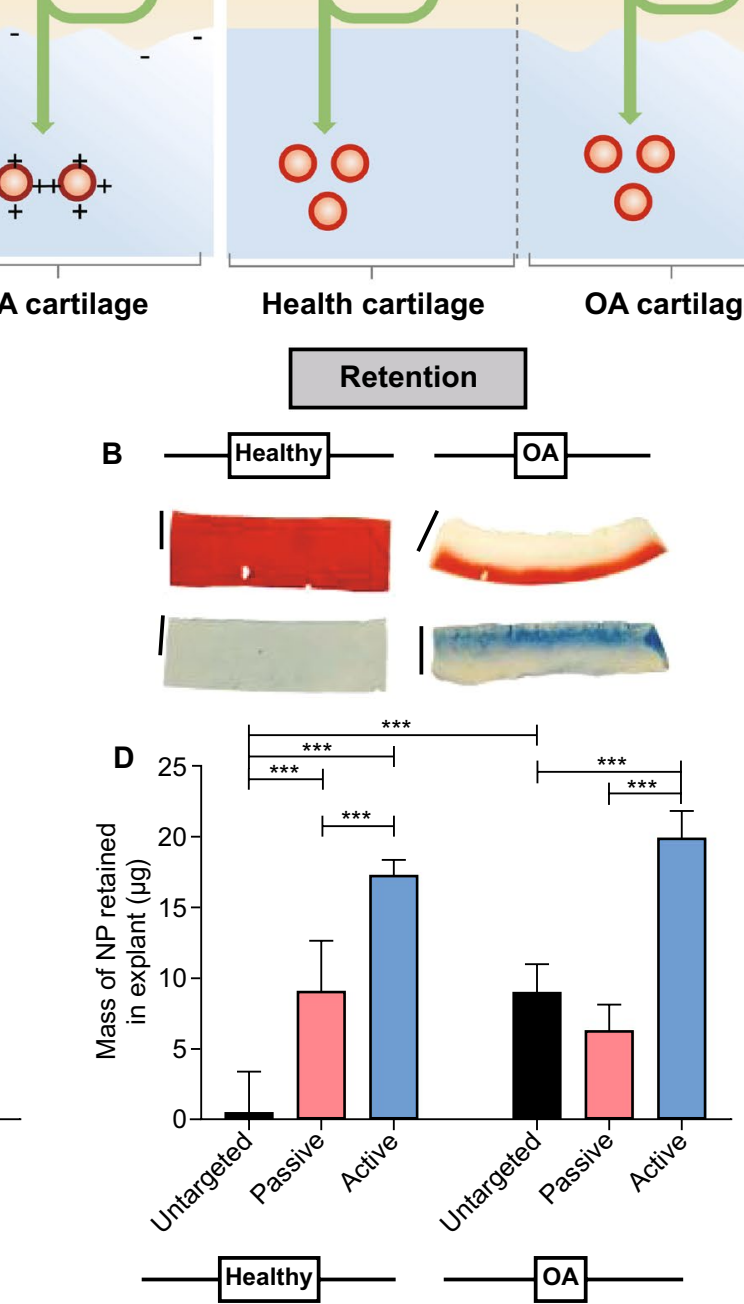

Fig. 4 Nanoparticle design for cartilage targeting therapy. a Strategies for passive and active targeting. b Effects of passive and active targeting on the penetrations of nanoparticles within healthy and OA cartilage. $\mathbf{c}$ Interactions of passive and active targeting nanoparticles with healthy and OA-mimicked cartilage. Reproduced with permission [54]. Copyright 2019, Acta Materialia Inc. Published by Elsevier Ltd. 


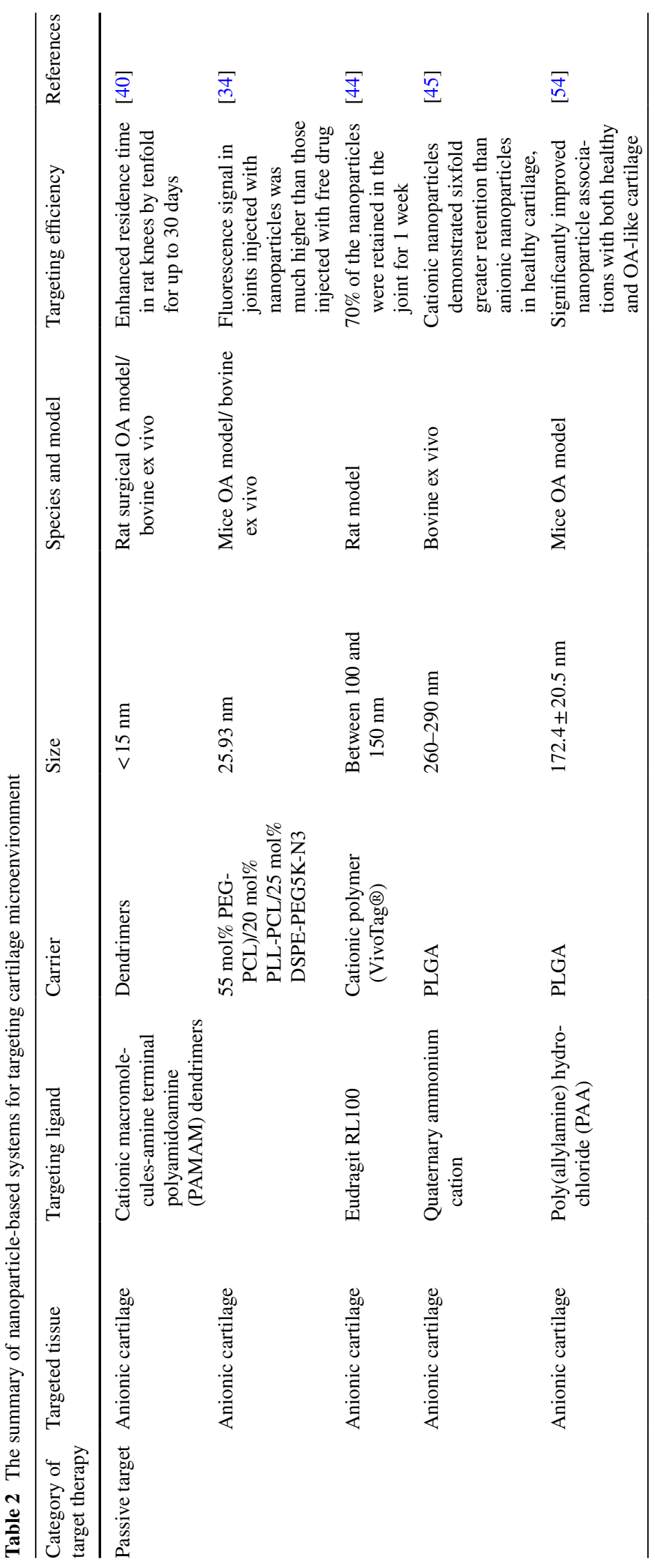




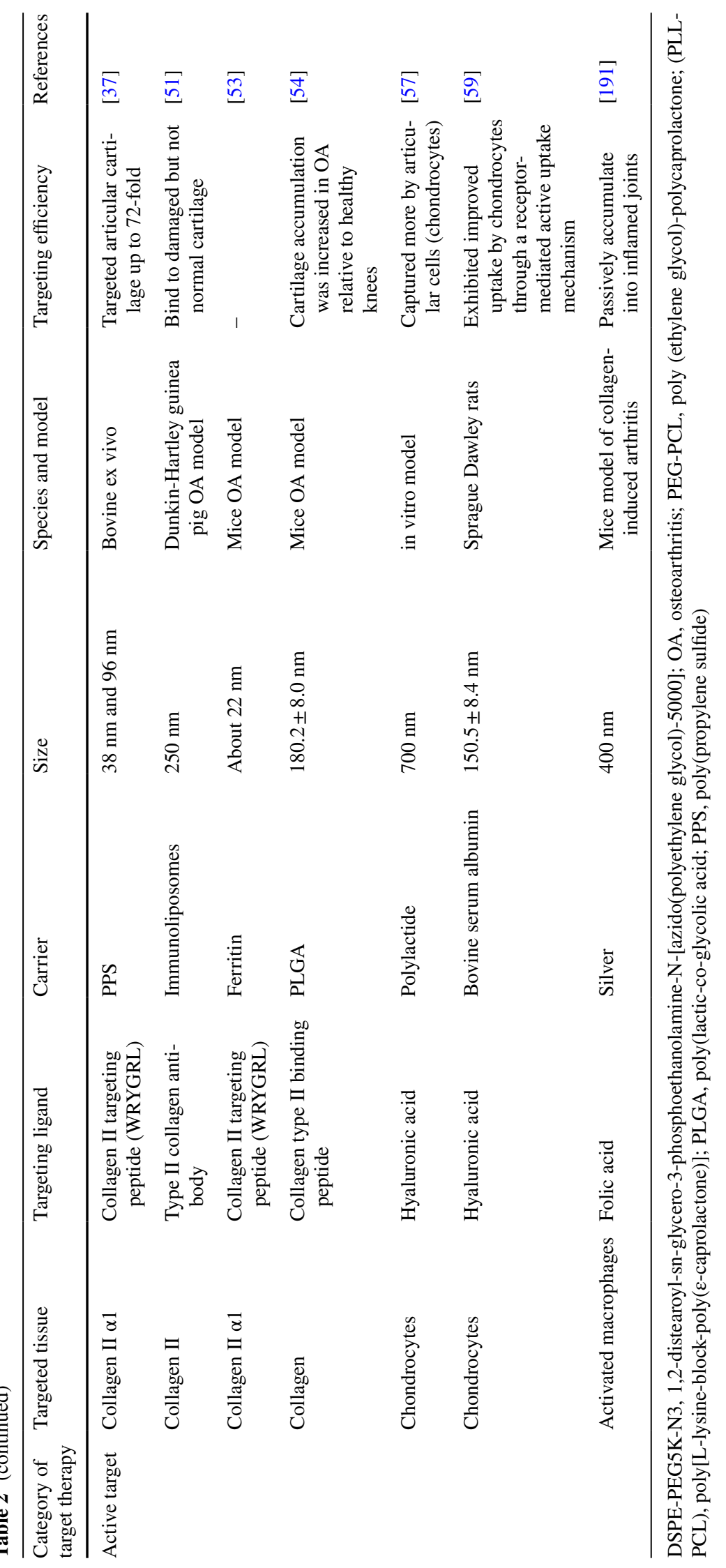


targeted cells (Fig. 5). For the indirect interaction, therapeutic agents can be released to the cartilage matrix and affect cellular communications via receptor ligand interactions. For the direct interaction, nanoparticles may enter the targeted cells by endocytosis-based uptake pathways. Nanoparticles are typically confined within intracellular vesicles, such as endosomes, phagosomes, or macropinosomes [60]. Endocytosis-based uptake pathways can be further categorized into phagocytosis, macropinocytosis, clathrin- and caveolaemediated endocytosis, and clathrin- and caveolae-independent routes, which are regulated and mediated by specific type of lipids and transport proteins [60, 61]. Most nanoparticles accomplish intracellular delivery by endocytosis and endosomal escape; in particular, intracellular gene delivery for in situ cellular reprogramming can be closely associated with endocytosis. Since nucleic-acid biomolecules are negatively charged, the penetration into cartilage ECM and diffuse across negatively charged phospholipid cell membranes become extremely difficult; as such, nanoparticles are developed to overcome the obstacles. The major existing approaches based on the platform of nanoparticles include intercellular delivery of transcription factors, RNA-based therapeutics and gene editing [62]. After being delivered in the cytoplasm, genes will directly regulate mRNA levels or translocated to the nuclei. Elucidate fundamental mechanisms of how nanoparticles gain access into chondrocytes are still critical for the mediation of physicochemical parameters, including size, charge, shape, and surface modifications) to increase therapeutic efficacy. Interactions of nanoparticles with targeted cells are also possibly affected by the severity of disease. Increased activities of catabolic enzymes in OA may negatively influence the indirect interaction with targeted cells by changing both properties of nanoparticles and physiological activities of the targeted cells. In addition,

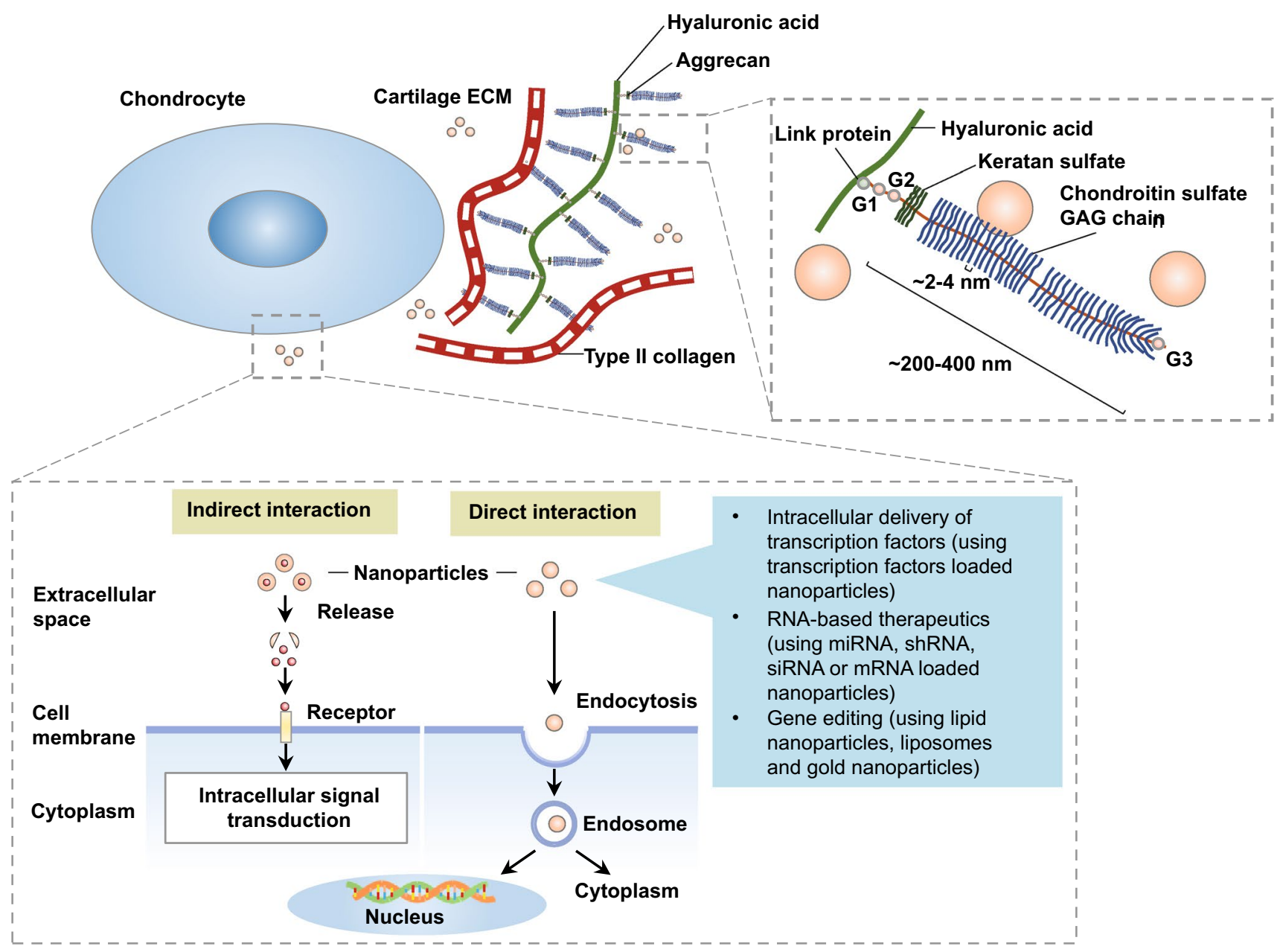

Fig. 5 Possible interactions of nanoparticles with targeted cells 
uptake pathways of nanoparticles in normal chondrocytes and diseased chondrocytes, including hypertrophic chondrocytes and, apoptotic chondrocytes, may be different and the mechanisms behind remain to be investigated.

\subsection{Summary of Size Design}

The optimal size of the nanoparticles should be designed according to the target sites for treatment. If the target sites are inflamed synovial fluid or synovial membrane rather than deep layer of cartilage, large sized, non-penetrating drug carriers can be used to avoid the clearance of blood vessels and lymphatics. If the target sites are superficial zones of cartilage, the size of nanoparticles should be at least smaller than the pore of collagen type II fibrillar network (50-60 nm) [36, 37]. After binding to the cartilage surface, nanoparticles can release the encapsulated drugs at deeper sites. If the target sites are full thickness of cartilage, the size of nanoparticles should be even smaller than the pore of proteoglycan network $(20 \mathrm{~nm})$ [37, 38]. In addition, active or passive targeting strategies can be used to reduce articular cavity clearance and increase the retention time within cartilage matrix.

\section{Materials Design of Nanoparticles}

Nanoparticles as delivery systems mainly contain transport carriers, bioactive elements and therapeutic agents (e.g., drugs and genes) (Fig. 6). Therapeutic agents are encapsulated by transport carriers, which are mainly responsible for controlling the pharmacokinetics of therapeutic agents including ensuring efficient concentration within cartilage while decreasing undesirable side effects. Bioactive elements are engineered for locally enhancing delivery efficiency and improving the cartilage microenvironment. Compositions of nanoparticles and their focused pathological pathways are summarized in Table 3.

\subsection{Major Compositions of Transport Carriers}

\subsubsection{Synthetic Polymers}

Biodegradable synthetic polymers such as PCL, poly (glycolic acid) (PGA), D, L-poly (lactic acid) (D, L-PLA), poly (1-lactic Acid) (PLLA), and their copolymer polylactic-coglycolic acid (PLGA) are frequently used biodegradable polymers. Because of the promising mechanical characteristics, high biocompatibility, and versatility of chemistry, some of them (e.g., PLA, PGA and PLGA) have been approved by the US Food and Drug Administration (FDA) and European Medicines Agency (EMA) as carriers for drug delivery in humans. Biodegradable synthetic polymers as nanocarriers for target delivery can increase bioavailability, protecting instable agents (e.g., proteins and genes), and minimizing toxicity effects. In addition, the production cost of synthetic polymer material is often lower than that of natural polymer material while the shelf time is longer.

PLA and PLGA have been widely used as drug delivery systems in animal studies of OA therapy [29, 57, 63, 64]. The major advantage of synthetic polymers is a good control over their physical and chemical properties. The surface properties can be tailored for specific biomedical applications. Because the negative charge on synthetic polymers (e.g., PLGA) surface may reduce the ability to enter the negative charged cartilage matrix, cationic surface modifications of PLGA-based nanoparticles by using cetyltrimethylammonium bromide, polyethyleneimine, poly(2-dimethylamino)ethyl methacrylate, didodecyldimethylammonium bromide, and chitosan is necessary for improving drug delivery efficacy [65-67]. Additionally, it is feasible to incorporate both hydrophilic and hydrophobic substances in synthetic polymers [68]. For example, surface modification of (hydrophobic) PLA and PLGA with hydrophilic PEG to form an amphiphilic block copolymer facilitates a high drug loading and efficient delivery within tissue [68]. Hydrophobic PLGA core can be used for encapsulation of drugs and genes, while the hydrophilic PEG shell prevents the surface from aggregation, opsonization, and phagocytosis and prolongs systemic circulation time [68, 69]. Moreover, the degradation speed can be manipulated to control the release of therapeutic agents. The hydrolytic degradation of PLGA in vivo depends on hydrolysis of the polymers to generate the lactic and glycolic acid monomeric components which can be tailored through controlling polymer molecular weight, copolymerization, and functionalization. By adjusting the size and structure, degradation kinetics of synthetic polymer nanoparticles can be controlled to achieve dosage- and site-specific drug delivery [63, 64, 70, 71]. However, the potential drawback is that their acidic 
Major compositions of transport carrier
Advantages

- Good biocompatibility:

Similar to GAGs

Cationic properties

Maintenance of chondrogenic phenotype

- Easy for surface modification

- Adjustable degradability

Advantages

- A better control over their physical and chemical properties: Controllable degradation kinetics Increasing bioavailability Stabilizing sensitive agents Minimizing side effects Easy for surface modifications

Limitations

- Potential adverse effects caused by acidic degradation products

\begin{tabular}{l|l} 
Bioactive elements derived from & $\begin{array}{l}\text { Synthe } \\
\text { polym }\end{array}$ \\
native cartilage ECM & $\begin{array}{l}\text { Hyalur } \\
\text { acid }\end{array}$ \\
Advantages \\
- $\quad$ Enhancing therapeutic efficacy \\
$\quad \begin{array}{l}\text { Targeting CD44-expressed } \\
\text { chondrocytes }\end{array}$ \\
- Providing viscoelastic properties \\
and improves joint biomechanics \\
$\quad \begin{array}{l}\text { Antioxidation, anti-inflammation } \\
\text { and analgesic effect }\end{array}$ \\
$\begin{array}{l}\text { Limitations } \\
\text { Potential pro-inflammation effects } \\
\text { caused by the degradation } \\
\text { products }\end{array}$
\end{tabular}

\section{Limitations}

- Poor water solubility

- Charge deduction at physiological pH

- Poor targeting and transfection efficiency

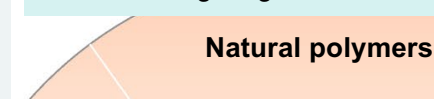

Natural polymers
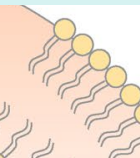

- Preparation process may damage

Therapeutic agents

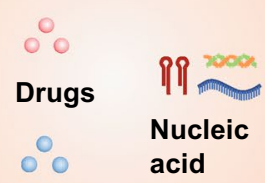

Grow

factors acid

Grow
factors
Chondroitin sulfate
- Enhantages
- $\quad$ Providing great compression resistance
- Increasing the production of hyaluronic
acid
Binding to chondrocytes
Anti-inflammatory and antioxidant
properties
proteinaceous drugs

- A rapid release of drug in the aqueous environment

Advantages

- Low toxicity and good biocompatibility

- Feasibility of surface modification

- As bio lubricants for friction reduction

imitations

Liposomes

Advantages

- Improving cartilage microenvironment

- As easily obtainable, renewable resource

- Manipulatable crosslinking degree

Limitations

- Immunoreactivity related to the source from non-human species

Fig. 6 Compositions and properties of biomimetic nanoparticles for the treatment of cartilage disease

degradation products, including caproic acid, succinic acid, valeric acid, and butyric acid as degradation product of PCL, may aggravate cartilage inflammation and matrix degradation.

\subsubsection{Natural Polymers and Their Derivatives}

Polysaccharides including chitosan, dextran, alginate, and cellulose derivatives are the most versatile natural polymers that broadly used in drug delivery. Among them, biologically active natural GAG analogues such as chitin and chitosan show therapeutic potential for inter-articular drug delivery. Their physical and chemical resemblance of cartilage ECM determines their major advantages-low toxicity and good biocompatibility. As the most abundant polysaccharide in the marine ecosystem and second in nature (after cellulose), chitin can maintain the morphology of chondrocytes and preserve the synthesis of ECM [72,73]. Chitosan (poly- $\beta-1,4$-linked glucosamine) as derivative conversed from alkaline $\mathrm{N}$-deacetylation of chitin has been widely studied for the delivery of therapeutic agents to cartilage [74]. The molecular structure of chitosan is similar to GAGs in normal cartilage, determining its good biocompatibility for maintaining the chondrogenic phenotype and proliferation activity $[75,76]$. The cationic property of chitosan 


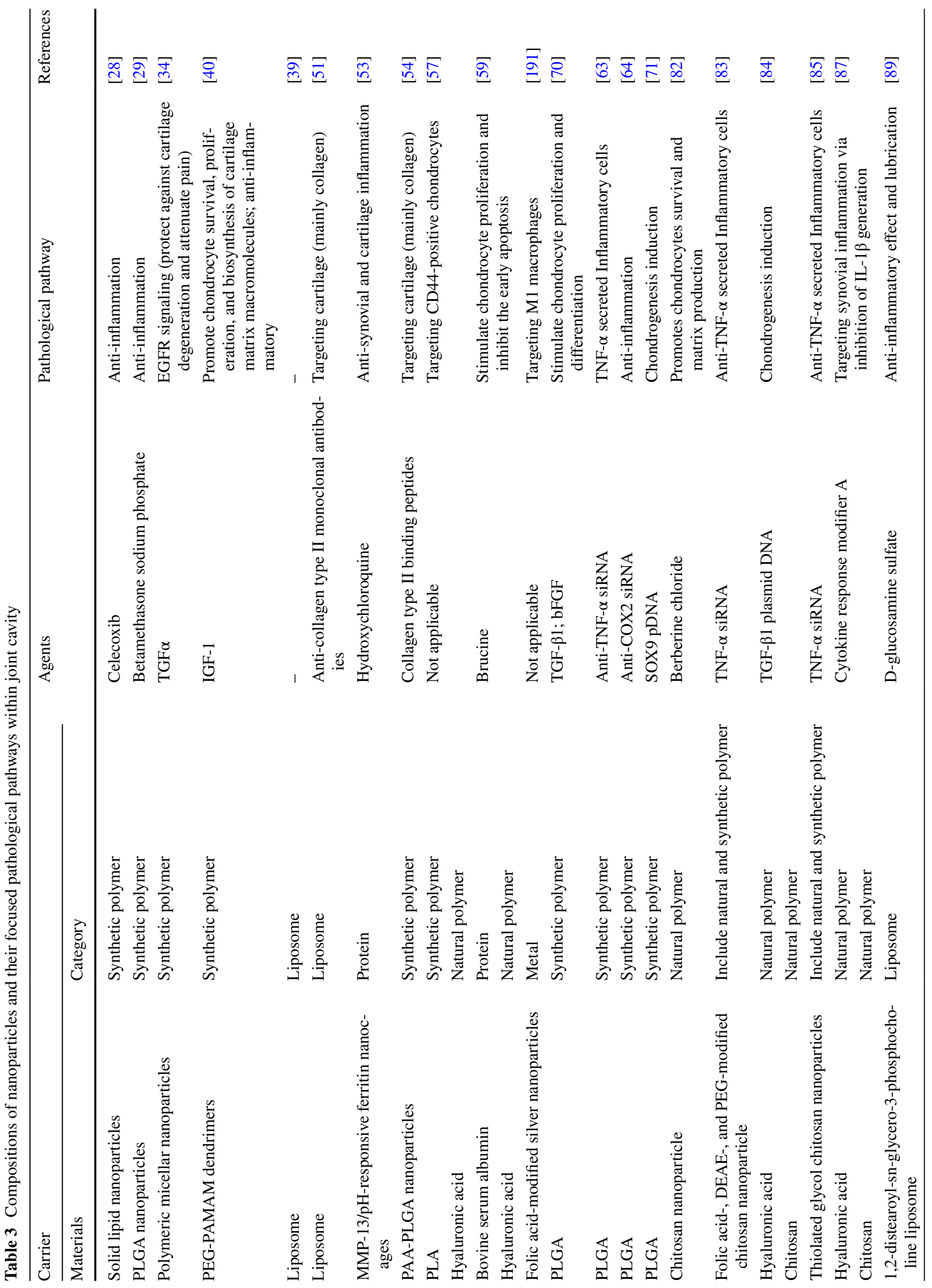




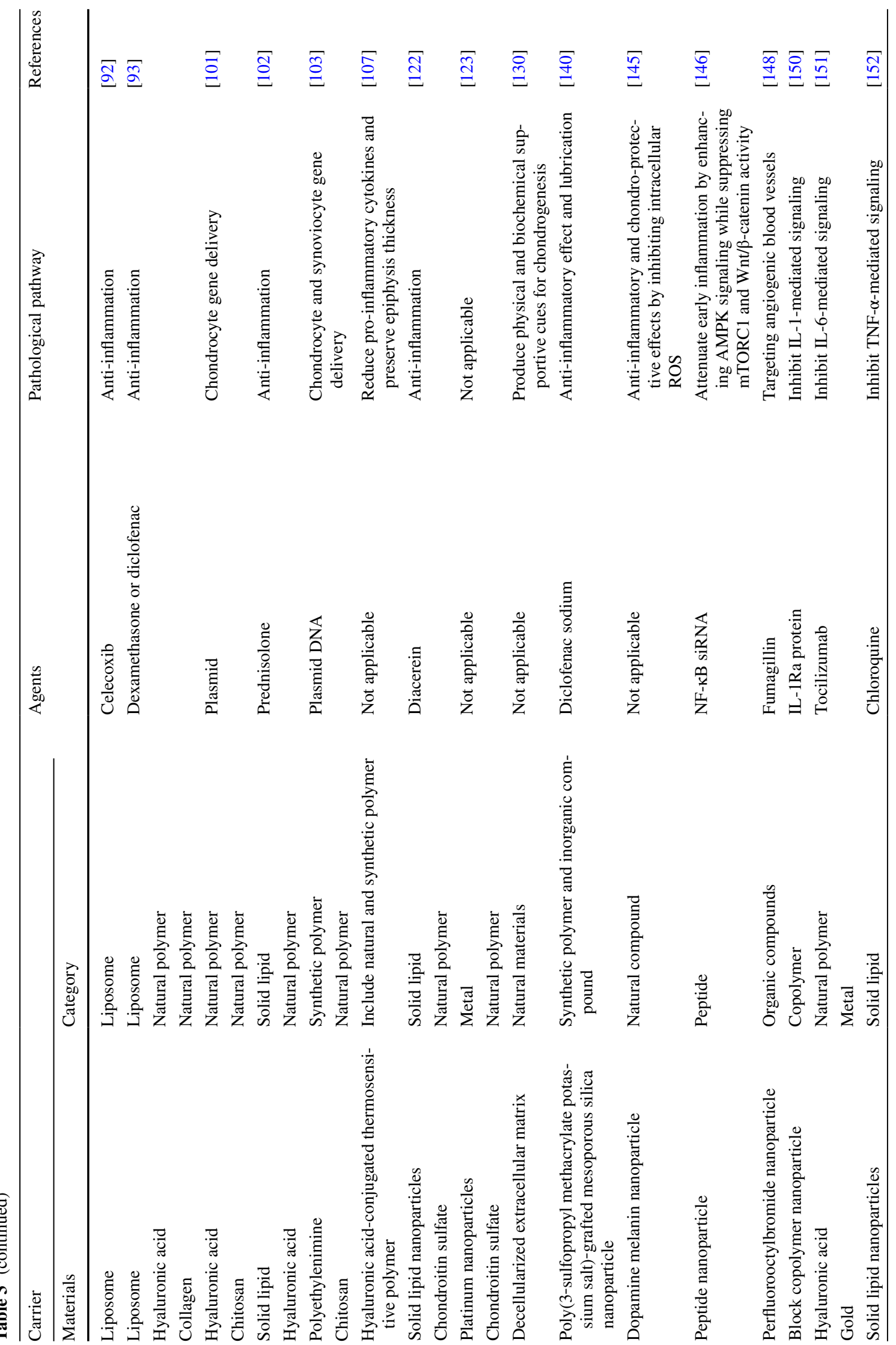




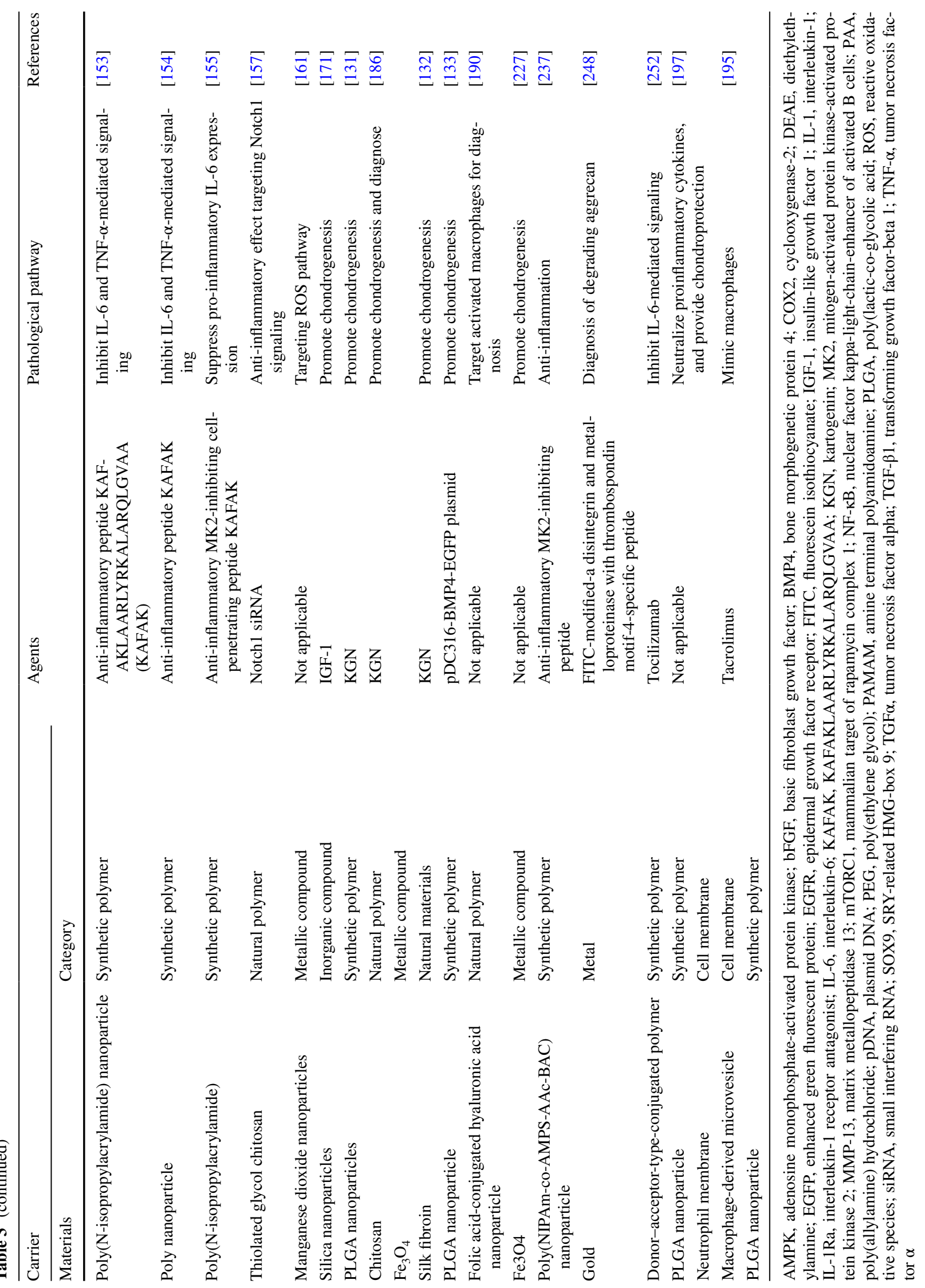


makes it different from most neutral or negatively charged polysaccharides. The positive charge allows it easily to bind to the negatively charged cartilage ECM or form electrostatic complexes with other negatively charged polymers. Because of the mild processing conditions and chemical reactivity, chitosan has been widely used in the field of surface modification [77]. The existence of $\beta-(1,4)$ glycosidic bonds between d-glucosamine and $\mathrm{N}$-acetyl-d-glucosamine provides possibilities to be modified for altering properties such as solubility, adhesion and stability [78]. Adjustable degradability is another property of chitosan determined by the deacetylation degree [79]. Lowly deacetylated chitosan degrades fast [79]. Besides, chitosan possesses non-toxicity, hydrophilicity, anti-inflammation, anti-bacterial and antifungal properties, and wound-healing effects [78].

Chitosan nanoparticles have been widely used as stable delivery systems for either controlled drug release or as a non-viral gene vector for transferring genes [80, 81]. For drug delivery, intra-articular injection of drug-loaded chitosan nanoparticles decreases the concentration of therapeutic agents in plasma, increases the retention time in synovial fluid, and therefore effectively ameliorates OA [82]. For gene delivery, the combination of chitosan nanoparticles with DNA or siRNA is able to transfect the chondrocytes [83-87]. The small size makes it more easily to be taken up by endocytosis of chondrocyte [26]. However, before further application, a series of problems need to be resolved such as poor water solubility, charge deduction at physiological $\mathrm{pH}$, and poor targeting and transfection efficiency [88].

\subsubsection{Liposomes}

Liposomes have been investigated as micro- or nanocarriers to change pharmacokinetics and biodistribution of drugs in the treatment of OA. Besides the low toxicity and good biocompatibility, liposomes can incorporate both hydrophilic and hydrophobic molecules and exist feasibility of surface modification to present targeting option and prolong the retention in cartilage. Phospholipids act as effective biolubricants for friction reduction and maintenance of mobility of synovial joints. Therefore, it is possible to achieve both sustained drug delivery and improved lubrication by using liposomes at the same time [89]. Liposomes of larger size display good retention in joint cavity and therefore are better for the improvement of joint boundary lubrication [90, 91]. Although the size (above $100 \mathrm{~nm}$ ) of liposomes determines the poor penetration within cartilage, sustained drug release within articular cavity can be provided through liposome dissolution. With a high encapsulation efficiency (as high as $90 \%$ ), anti-inflammatory drug-loaded liposomes displays more promising outcome than the therapeutic entities they contain in pain control and cartilage protection [92, 93]. Nevertheless, the preparation procedure contains the mix with organic solvents which may damage proteinaceous drugs. Additionally, the aqueous environment of the synovial fluid may lead to a rapid burst release of drug.

\subsection{Components Derived from Native ECM}

The compositions of natural ECM provide templates for the selection of bioactive and biomimetic materials. Mainly produced by chondrocytes, ECM is composed of collagens (60-85\% of dry weight), proteoglycans (15-40\% of dry weight), and other non-collagenous proteins, and responsible for retaining water and maintaining mechanical properties that are anisotropic, nonlinear, inhomogeneous and viscoelastic (Fig. 4) [94]. Type II collagen is the principal collagen ( $90 \%$ to $95 \%$ of collagen) in ECM, and the fibers are intertwined with proteoglycan aggregates $[94,95]$. Proteoglycan aggregates are high molecular weight molecules which are composed of GAGs covalently bound to a central protein [94, 95]. Type II collagen and proteoglycans are mainly responsible for the tensile and compression strength, respectively [94, 95]. GAGs as high molecular weight linear polysaccharides can be divided into four classes including hyaluronic acid, keratan sulfate, dermatan sulfate, and chondroitin sulfate $[94,95]$. Aggrecan is the main proteoglycan that its core protein contains three globular domains and two glycosaminoglycan-attachment domains [95]. An N-terminal globular domain of aggrecan interact with hyaluronic acid to form proteoglycan aggregates [95]. The chondroitin sulfate chains attach to the chondroitin sulfate domain, which is responsible for the high fixed charged density and the ability to resist compressive loads in cartilage [95]. Chondrocytes receive nutrients depending on the diffusion of synovial fluid and also indirectly interact with components of synovial fluid including hyaluronic acid, lubricin, glucose, aggrecan, chondroitin sulfate, keratan sulfate, and water [96]. 


\subsubsection{Hyaluronic Acid}

As a non-sulfated glycosaminoglycan, hyaluronic acid maintains a constant concentration in cartilage and synovial fluid as a space filler [97]. In synovial fluid, hyaluronic acid functions in lubrication, hydration balance, matrix structure, and steric interactions to provide viscoelastic properties [97]. The binding to ECM molecules and cell surface receptors makes hyaluronic acid as a modulator of cellular behaviors including differentiation, proliferation, development, and recognition [98]. When OA occurs, the decreased average molecular weight and concentration of hyaluronic acid aggravates damage to the cartilage [99].

Balazs and Denlinger proposed the concept of viscosupplementation for the treatment of OA [100]. The intra-articular injection of hyaluronic acid can recover the rheological properties of synovial fluid which further promote the synthesis of endogenous hyaluronic acid and consequently improve joint biomechanics [100]. Hyaluronic acid also exerts pharmacologic actions including antioxidation, antiinflammation, analgesic effect and chondroprotection [97]. Hyaluronic acid has emerged as the moiety of the drug and gene carriers for OA treatment [86, 87, 101, 102]. Multiple functional units in hyaluronic acid enable it to be chemically modified by other moieties which is beneficial for enhancing therapeutic efficacy or decreasing toxicity. For gene delivery, the chondrocyte transfection efficiency of nanoparticles with hyaluronic acid is higher than that without hyaluronic acid $[101,103]$. CD44, as a receptor of hyaluronic acid, is also highly expressed by synovial lymphocytes, macrophages, and fibroblasts in inflamed joints [104-106]. Hyaluronic acid-based nanoparticles can carry anti-inflammatory drugs targeting these cells as an active targeting strategy. Hyaluronic acid-decorated nanoparticles can also target CD44expressed chondrocytes and therefore lead to better targeting in cartilage [58]. Besides improving chondrocytes targeting efficiency, hyaluronic acid-based nanoparticles persist longer retention than free drugs and those without hyaluronic acid [102]. More interestingly, hyaluronic acid with the conjugation of a thermosensitive polymer displays spontaneous formation of nanoparticles after intra-articular injections to a murine OA model. Those nanoparticles offer a prolonged residence time (exceed 21 days near the injection site) to reduce pro-inflammatory cytokines and preserve epiphysis thickness [107]. Nevertheless, it should be noted that larger hyaluronic acid molecules are depolymerized producing low molecular weight hyaluronic acid, leading to excess inflammatory response [108].

\subsubsection{Chondroitin Sulfate}

As highly sulfated and linear polysaccharide, chondroitin sulfate makes up the main constituent of GAGs, accounting for $20 \%$ weight/dry weight of adult articular cartilage [109-113]. Chondroitin sulfate is composed of a chain of alternating sugars ( $\mathrm{N}$-acetylgalactosamine and glucuronic acid) and has an important role in maintaining the structural integrity of cartilage [110,111]. The turnover of chondroitin sulfate affects the mechanical property of ECM and modulates the homeostasis of chondrocytes. Disaccharide unit heterogeneity and sulfates on disaccharide units determine the negative charge of chondroitin sulfate polymer and its biological activities in cartilage such as the maintenance of the water content and the great resistance to compression [114]. Chondroitin sulfate increases the production of hyaluronic acid by synoviocytes to maintain viscosity [115]. The capacity to bind chondrocyte is 5- to 7-times higher than hyaluronic acid and keratin sulfate [116]. In addition, chondroitin sulfate inhibits the synthesis and activities of proteolytic enzymes, nitric oxide, and other substances and thus prevent cartilage matrix from damage [117]. Chondroitin sulfate can reduce the nuclear translocation of NF- $\mathrm{KB}$ to inhibit inflammation, favor the synthesis of hyaluronic acid and collagen II, and therefore limit matrix degradation $[117,118]$. In OA, the degradation of chondroitin sulfate in cartilage is increased, which further increases water content in cartilage ECM to induce a hypertrophy-like morphology of chondrocytes and MMP-13/ADAMTS5 production [119, 120]. The European League Against Rheumatism (EULAR) gave chondroitin sulfate the highest recommendation for the treatment of OA [121]. Although the oral drug delivery has been commercialized, there is still challenge in securing instability of delivery system to achieve its efficacy. As the moiety of nanoparticles, chondroitin sulfate displays the potential to increase delivery efficiency in joints while without leading to toxicity $[122,123]$. The hydrophilicity property of chondroitin sulfate-based nanoparticles increases water solubility of hydrophobic drugs, prolongs articular cavity retention, and promotes cartilage targeting $[122,123]$. 


\subsubsection{Collagen and Acellular ECM}

As the most abundant biopolymer in the human, collagen becomes an easily obtainable, renewable resource for the recovery of cartilage ECM structure and function. Besides the capacity to resemble the cartilage microenvironment, collagen exhibits an extremely high biocompatibility with low immunogenicity and is biodegradable and bioresorbable. Type II collagen can minimize chondrogenic hypertrophy, prevent joint destruction and pain for the treatment of OA [124, 125]. Moreover, the degree of cross-linking can be manipulated and the physical properties such as size, surface area, and absorption capacity, are easy to configure, which makes collagen-based nanoparticles a prime candidate for controlling drug release. Commercial native collagen products have been extracted from chicken sternum [124]. One potential drawback, however, is the immunoreactivity related to the source from non-human species.

Acellular ECM which theoretically contains all the bioactive compositions is the nature's template to provide adequate nutrient support for tissue repair [126, 127]. Therefore, acellular ECM is biodegradable and do not elicit adverse immune responses. The properties to induce chondrogenic differentiation and promote cartilage regeneration have been proved [128, 129]. As the major component of nanoparticles, it is capable of supporting viability and proliferation of chondrocyte [130].

\subsection{Intra-articular Delivery Choices}

At present, most of the basic research focuses on the direct intra-articular injection of nanoparticles to solve the clinical problem of rapid drug clearance. Direct intra-articular injection of nanoparticles can minimize systemic exposure and increase local bioavailability by providing controlled and sustained drug release. However, clinical intra-articular drug injection is used in most cases to treat mild to moderate OA. Attempts to use nanoparticles for the treatment of severe OA are more desirable. If nanoparticles can be accumulated more at the severely defective sites, the therapeutic outcome could be better than that evenly distributed in the articular cavity. The combination of hydrogels or scaffolds with nanoparticles can enhance the stability of nanoparticles and extend the retention of drugs following intra-articular injection [131-133] (Table 4). In addition, scaffolds or hydrogels can affect cell survival and provide matrix for cell homing and regeneration [131-136].

Hydrogels consisting cross-linked hydrophilic polymers for retaining water can mimic three-dimensional structure of the ECM and thus improve lubrication. With good biocompatibility and high permeability for nutrients, hydrogels can fill cartilage defects of any size in a minimally invasive way. Various hydrogel systems containing nanoparticles have been reported in the literature for articular cartilage repair [70, 137]. However, low mechanical strength is a major shortcoming of hydrogels. Since loading patterns affect the diffusion process of therapeutic agents [138], therapy based on nanoparticles alone is difficult to provide enough mechanical support at the region of severe wear and tear. The combination with scaffold is a strategy to solve the problem especially for the repair of large defect in weightbearing area. An ideal biodegradable scaffold should favor cell survival and alleviates the further wear and degradation of the cartilage to support the biomechanical environment, which in turn provides a proper microenvironment for the controlled release of nanoparticles. In addition, nanoparticles can also provide support for the stability of hydrogels and scaffolds. For example, laponite nanoparticles can construct an interpenetrating network which enhances the hydrogel mechanical properties [137]. Further, the combination of nanoparticles with scaffolds or hydrogels is possible to achieve dosage- and site-specific multiple drug delivery [70].

\subsection{Summary of Material Design According to the Pathology Features}

Since each material has its own characteristics, the adequate combination of different materials can improve the efficiency of drug delivery while circumvent individual shortcomings. The material selection needs to be considered for its own characteristics and the difference in target sites during the pathological processes. For example, liposomes with larger size are more suitable for articular cavity drug delivery and viscosupplementation for prevention of OA. In the next section, we discuss the therapeutic mechanism of nanoparticles during the pathological progress of OA. 


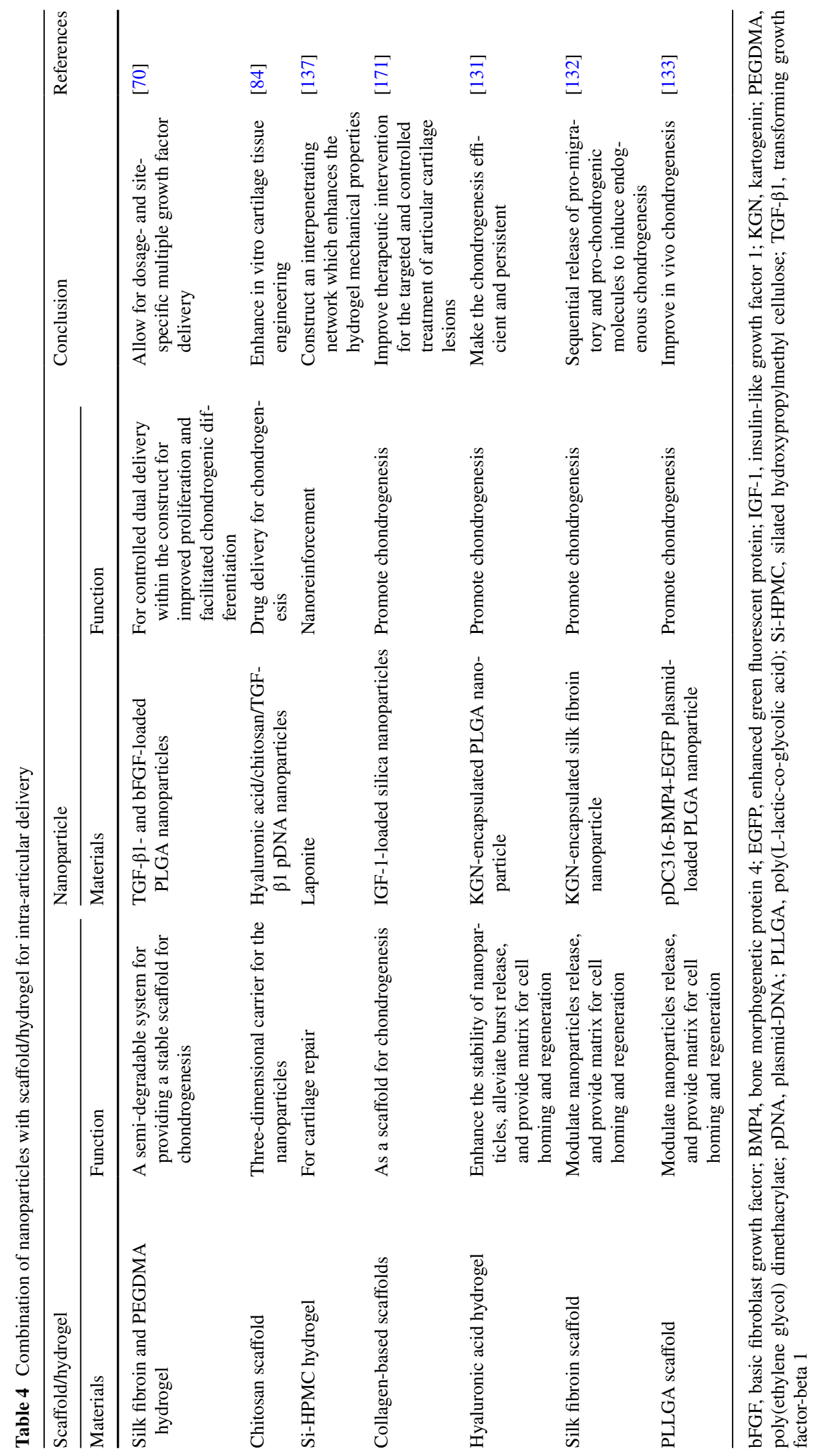


(a) Viscosupplementation

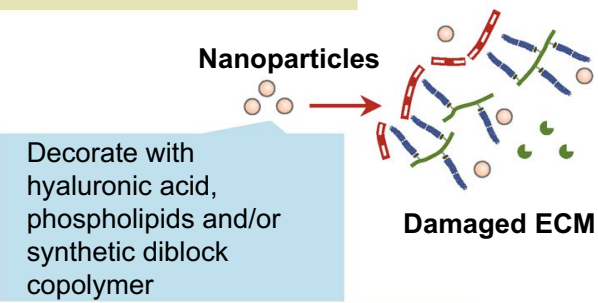

(c) Improving chondrocytes survival

Anti-inflammation, antioxidant stress, preventing chondrogenic hypertrophy and regulating autophagy

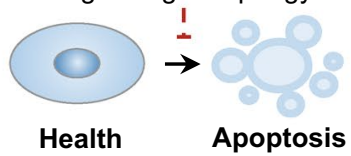

(b) Minimizing chondrogenic hypertrophy

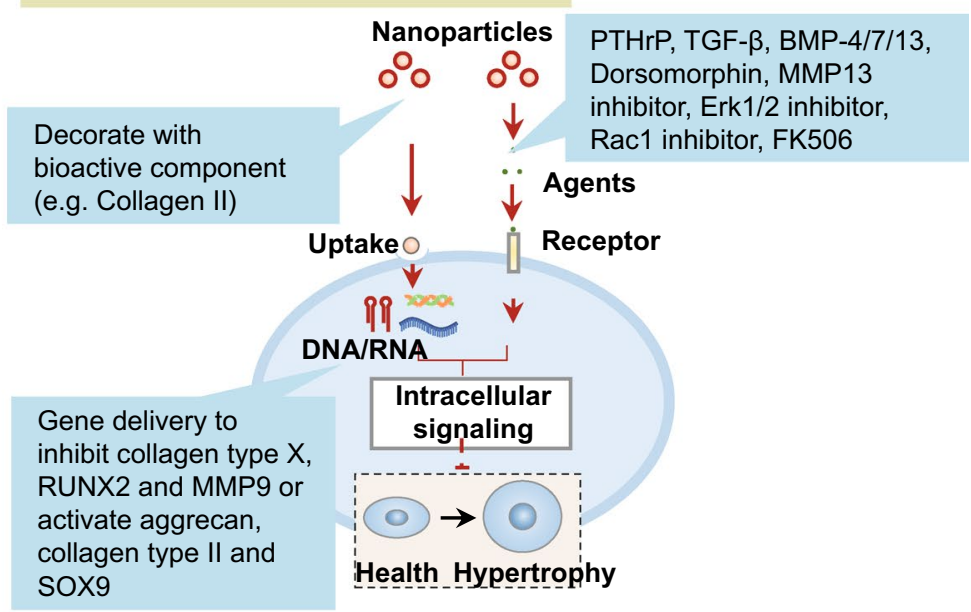

(d)

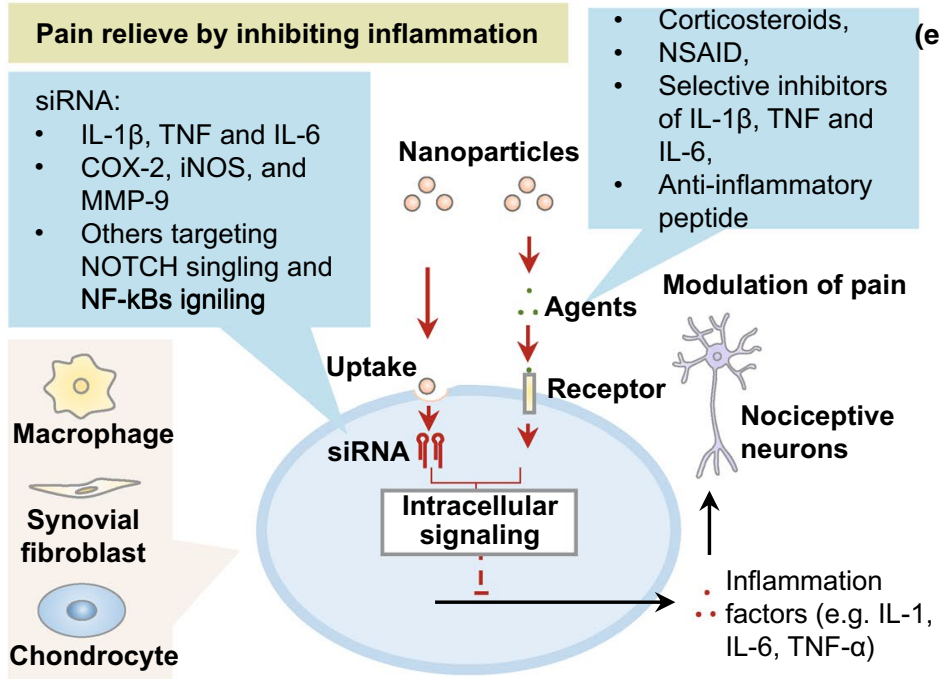

(e) Protecting cartilage against oxidative damage

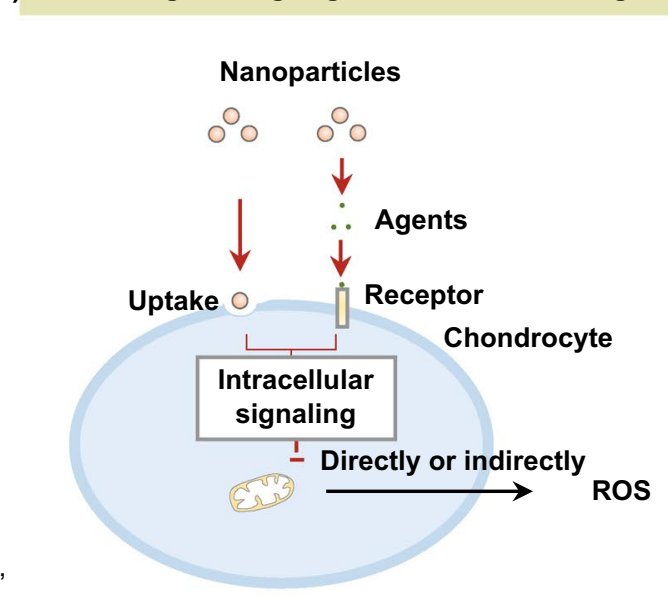

(f) Recruitment of endogenous cell

Synovium-
derived MSCs
synovial fluid-
derived MSCs
Fatem cells
stem/progenitor cells
Bone marrow
derived MSCs
MSCs in Ranvier
groove

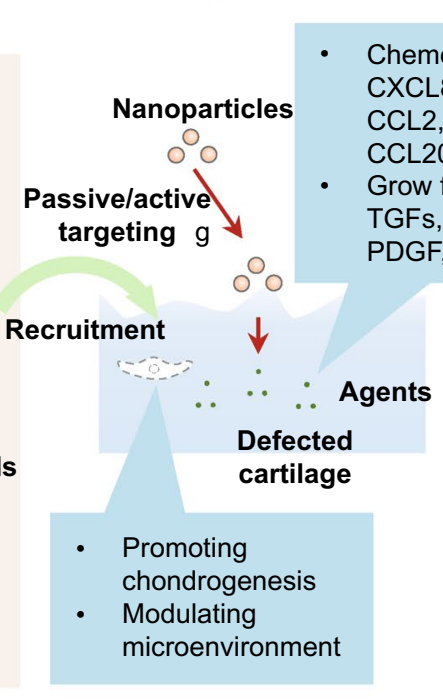

(g) Promoting chondrogenesis

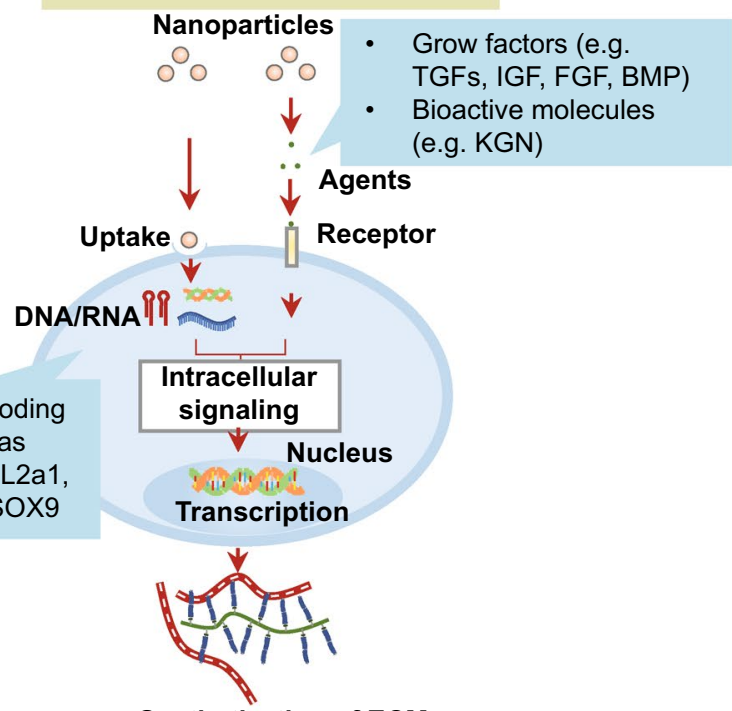

Synthetization of ECM 
4Fig. 7 Uptake pathways and therapeutic mechanisms of nanoparticles in OA. The major mechanisms include a lubrication improvement, b chondrogenic hypertrophy prevention, c cell survival regulation, $\mathbf{d}$ pain relief by inflammation inhibition, $\mathbf{e}$ anti-oxidative damage, $\mathbf{f}$ recruitment of endogenous stem cells, and $\mathbf{g}$ chondrogenesis promotion. Abbreviations: ACAN, aggrecan; BMP 4/7/13, bone morphogenetic proteins 4/7/13; CCL 2/3/20, C-C motif chemokine ligand 2/3/20; COL2a1, collagen type II alpha 1 chain; COX 2, Cyclooxygenase-2; CXCL 8/12, chemokine (C-X-C motif) ligand 8/12; Erk1/2, extracellular signal-regulated protein kinase 1/2; FGF, fibroblast growth factors; FK506, tacrolimus; IGF, Insulin-like growth factor; IL $1 \beta / 6$, Interleukin $1 \beta / 6$; iNOS, inducible nitric oxide synthase; KGN, kartogenin; MMP 9, matrix metalloproteinases; NF- $\kappa B$, nuclear factor kappa-B; NSAID, nonsteroidal anti-inflammatory drugs; PDGF, Platelet-derived growth factor; PTHrP, parathyroid hormone-related protein; Rac1, Ras-related C3 botulinum toxin substrate 1; ROS, reactive oxygen species; SOX 9, SRY-Box transcription factor; TGFs, transforming growth factors; TNF, tumor necrosis factor

\section{Therapeutic Schemes According to the Pathology Mechanisms}

\subsection{Prophylactic Administration}

\subsubsection{Viscosupplementation}

OA progress is associated with lubrication deficiency caused by the age-related degradation of glycoprotein (i.e., hyaluronic acid) [96, 139]. Nanoparticles combining lubrication protect against OA (Fig. 7a). As mentioned above, nanoparticles with components such as hyaluronic acid and phospholipids all are conductive to lubrication improvement and the maintenance synovial joint mobility. Synthetic diblock copolymer to mimic the functional domains of lubricin is also possible to be components of nanoparticles for lubrication improvement [139]. Polyelectrolyte polymer brushes can reduce friction coefficient via the hydration lubrication mechanism [140]. The combination with nanoparticle represents an effective approach to enhanced lubrication capability [140].

\subsubsection{Cartilage Maintenance by Minimizing Chondrogenic Hypertrophy}

Hypertrophic chondrocytes with a significant increase in cell size and volume degrade their surroundings and therefore accelerate the progression of OA [141]. The high expression of osteogenic differentiation-related genes appears to be associated with production of mineralized ECM proteins and calcification of articular cartilage [141]. Prevention of hypertrophy is a potential therapeutic strategy to facilitate the retardation of osteophytes and slow down OA progression (Fig. 7b).

High expressions of collagen type $\mathrm{X}$, runt-related transcription factor 2 (RUNX2), and MMP13 are the major characterization of hypertrophic chondrocytes [141]. Meanwhile, the expressions of hyaline cartilage markers such as aggrecan, collagen type II, and SOX9 are decreased [141]. Although there is lacking attempt by using nanoparticles for preventing hypertrophy, these markers provide references for the selection of gene or drug delivery targets. Current evidence indicates that NK3 homeobox 2 (Nkx3.2), mothers against decapentaplegic homolog (Drosophila) 6 (SMAD6), HDAC4, Chondromodulin 1/soluble Flt-1 and ETS-related gene (ERG) /C-1-1are also potential gene delivery targets to prevent chondrogenic hypertrophy [142]. Moreover, other developed therapeutic agents, including PTHrP, TGF- $\beta$, BMP-4/7/13, Dorsomorphin, MMP13 inhibitor, Erk1/2 inhibitor, Rac1 inhibitor and FK506, can be employed in combination with nanoparticles for delivery [142]. More importantly, by the adding above discussed bioactive ECM compositions (e.g., collagen), the efficacy of preventing hypertrophy can be further improved [125].

\subsubsection{Cartilage Maintenance by Improving Chondrocytes Survival}

Chondrocyte survival is important for the maintenance of cartilage matrix. Nanoparticles have been utilized to inhibit apoptosis (Fig. 7c). For example, berberine-loaded chitosan nanoparticles can effectively improve cell survival and ameliorate OA [82]. In fact, anti-inflammation, antioxidant stress, and the prevention of chondrogenic hypertrophy are all beneficial for cell survival. Adequate autophagy regulation is another strategy to promote chondrocyte survival. Normal chondrocytes express high levels of autophagy promoted by adenosine monophosphate-activated protein kinase (AMPK) and Sirtuin 1 to remove damaged and dysfunctional organelles and proteins [143, 144]. In OA, reduced autophagy in chondrocytes leads to increased catabolic processes and cell death [6, 143, 144]. Some nanoparticles have been fabricated to activate autophagy to mediate ROS scavenging, which is beneficial for relieving OA symptom [145]. Yan et al. fabricated the cationic amphipathic peptide-based nanoparticles for siRNA delivery in a mouse OA model 
[146]. By suppressing NF- $\mathrm{BB}$, chondrocyte autophagic activity can be promoted through inhibiting mammalian target of rapamycin (mTOR), demonstrating its ability to permeate the dense cartilage matrix and treat OA [146].

\subsection{Symptomatic Treatment}

\subsubsection{Pain Relief by Inhibiting Inflammation}

Pain is the predominantly symptoms of OA, caused by the activation of nociceptive pathways [147]. Inflamed tissues in OA express proalgesic molecules such as nerve growth factor (NGF), bradykinin receptors and tachykinin which is responsive for pain activation [147]. Therefore, pain management for allaying OA symptoms is dominated by the inhibition of articular inflammation. Nanoparticles can enhance the therapy efficiency of anti-inflammatory drugs, such as steroid and nonsteroidal anti-inflammatory drug (NSAID) (Fig. 7d) [140, 148]. IL-1 $\beta$, TNF, and IL-6 modulate disturbed metabolism and enhanced catabolism in OA joint [149]. Nanoparticles loaded with selective inhibitors block these proinflammatory cytokines' production and counteract the degradation of cartilage in OA [122, 150-152]. Currently used selective inhibitors include diacerein (against IL-1), interleukin-1 receptor antagonist (against IL-1), tocilizumab (against IL-6), and chloroquine (against TNF- $\alpha$ ) [122, 150-152]. Besides that, cell-penetrating anti-inflammatory peptide KAFAKLAARLYRKALARQLGVAA (KAFAK) with the ability to suppress IL-1, TNF- $\alpha$, and IL-6 has also been used in combination with nanoparticles [153-155].

Nanoparticles-based gene delivery to interrupt unnecessary gene expression in specific target cells, such as macrophage and chondrocyte, is also promising to inhibit joint inflammation. siRNA-loaded nanoparticles directly inhibit the expression of inflammatory factors such as $I L-1 \beta, T N F$, and $I L-6$ [63]. IL-1 $\beta$ and TNF- $\alpha$ can promote the expression of cyclooxygenase-2 (COX-2) and inducible nitric oxide (iNOS) synthase, leading to the production of prostaglandin E2 (PGE2) and nitric oxide (NO). siRNA delivery to silence the expression of inflammation-related genes such as COX-2, iNOS, and MMP-9 is another strategy to suppress inflammation-associated catabolism [64, 156]. Besides, gene delivery targeting some signaling, such as NOTCH and NF- $\mathrm{BB}$, is also possible to contribute to inflammation inhibition, resulting in retarded cartilage damage and bone erosion [146, 157].

\subsubsection{Against Oxidative Damage}

In OA, mitochondrial dysfunction leads to excessive production of reactive oxygen species (ROS) and downregulation of antioxidants such as superoxide dismutase, catalase, and glutathione peroxidase [6]. Excessive production of ROS increases apoptosis in chondrocytes by increasing mitochondrial DNA (mtDNA) damage, which further results in ECM degradation and joint inflammation [158]. Antioxidant supplements, mediators of various ROS pathways, and free radical scavengers are being investigated to protect cartilage against oxidative stress damage $[159,160]$. Therapeutic agents-loaded nanoparticles have been designed to directly or indirectly protect cartilage against oxidative stress damage (Fig. 7e) [145, 161]. For example, manganese dioxide can catalyze the breakdown of hydrogen peroxide $\left(\mathrm{H}_{2} \mathrm{O}_{2}\right)$, a key radical that is derived from $\mathrm{O}^{2-}$ to downregulate $\mathrm{ROS}$ level [161]. In a rat model, manganese dioxide nanoparticles with suitable physicochemical properties (less than $20 \mathrm{~nm}$ and cationic) can address issues of rapid release and achieve cartilage protection [161].

\subsection{In situ Cartilage Regeneration}

With the progression of the OA and chondroptosis, a major challenge for attenuating the progression of cartilage degradation is the inability of the resident chondrocytes to lay down a new matrix $[6,162]$. Cartilage does not regenerate due to the hostile local microenvironment and a limited supply of endogenous cells; as such, the rapid recruitment, migration, and infiltration of more joint-resident endogenous stem cells can provide better outcomes. Ideally, these recruited stem cells will be stimulated to differentiate into chondrocytes along with matrix synthesis to achieve in situ cartilage regeneration. In situ cartilage regeneration mainly contains two steps-recruitment of endogenous stem cells and chondrogenic differentiation (Fig. 7f, g). 


\subsubsection{Recruitment of Joint-resident Endogenous Stem Cells}

Several types of MSCs including chondroprogenitor cells, synovium-derived MSCs, synovial fluid MSCs, bone marrow MSCs, fat pad-derived stem cells and MSCs in Ranvier groove are potential candidates for the recruitment into the defected cartilage [163]. The goal of recruitment is possible to be achieved by using nanoparticles decorated with advantageous cytokines or other active molecules (Fig. 7f). Cytokines including chemokines (e.g., CXCL8, CXCL12, CCL2, CCL3, CCL20) and grow factors (e.g., TGFs, IGF, FGFs, PDGF, BMPs) have been reported to facilitate MSCs homing and migration within cartilage [163]. Nanoparticles can be targeted to defect areas following active or passive delivery. With the degradation of nanoparticles and the release of cytokines, endogenous progenitor and stem cells can be recruited to synthesize and deposit nascent proteins and remodel the local microenvironments. Studies in future should fully consider the unfavorable effects of chemokines including activated inflammation, aggravated ECM catabolism, impeded chondrogenic differentiation, induced apoptosis and developed pain symptoms [163]. Moreover, it is also important to consider both the pathological mechanisms of $\mathrm{OA}$ and heterogeneity of stem cells. Biophysical and biochemical characteristics of nanoparticles should be designed to recruit specific subset of stem cells according to the pathological features.

\subsubsection{Promoting Chondrogenesis}

TGF- $\beta$ family plays a critical role in skeletogenesis and OA progression. TGF- $\beta 2$ or TGF- $\beta 3$ deficient mice displayed skeletogenesis defects in the forelimbs, hindlimbs, and craniofacial bones [164]. In a chondrocyte-specific $T g f b r 2$ knockout mice, higher expression of Runx2, Mmp13, Adamts5, and Col10 along with increased hypertrophic chondrocyte numbers, early osteophyte formation, and increased subchondral bone mass are found, resembling the process of OA development $[165,166]$. TGF family has been the most popular and widely investigated grow factor for cartilage repair. Intra-articular delivery TGF- $\beta$-loaded nanoparticles have been proved to stimulate ECM production, downregulate matrix-degradation, form hyaline cartilage, and therefore attenuate $\mathrm{OA}$ progression $[70,167,168]$.

IGF-1 decreases in spontaneous OA, which aggravates articular cartilage lesions $[169,170]$. IGF-1-loaded nanoparticles have also been successfully used to enhance cartilage repair and promisingly inhibit the progress of OA [40, 171]. IGF-1 promotes chondrogenesis of mesenchymal stem cell (MSC) and mediate chondrocyte phenotype [172, 173]. In addition, IGF-1 not only enhances the synthesis of proteoglycans and collagen type II, but also inhibits the ECM degradation by decreasing the production of matrix metallopeptidase [174, 175].

FGF-18 is a well-known anabolic growth factor which is involved in chondrogenesis and beneficial for cartilage repair [176]. Upregulation of FGF-18 induces the formation of cartilage with increased synthesis of matrix, and the in vivo delivery relieves the symptom of OA and promotes cartilage repair [176, 177]. $F G F-2$ deletion induces accelerated spontaneous and surgically induced OA which can be reversed by subcutaneous administration of recombinant FGF-2 [178]. However, controversial roles have been reported that FGF-2 exerts catabolic effects that displays by the upregulated matrix-degrading enzyme production and down regulated ECM accumulation [179-181]. Therefore, the pharmacological actions and mechanisms should be fully confirmed before the therapy application. The BMPs as a family of growth factors have been widely applied for bone regeneration, while the promotion of chondrogenesis is another property $[182,183]$. Both Smad-dependent and Smad-independent BMP pathways are required for chondrogenesis, and Indian hedgehog (IHH)/parathyroid hormonerelated protein (PTHrP) and FGF pathways are key downstream targets [184]. It is possible to apply BMPs to improve both cartilage and subchondral bones functions. Of note, to date, there is lacking attempt by using nanoparticles to delivery FGFs and BMPs for either preventing or treating OA. Further preclinical experiments are required to investigate the feasibility and efficacy of these novel approaches.

Kartogenin (KGN) as a small bioactive molecule to promote chondrogenic differentiation of stem cells was first reported by Johnson et al. in 2012 [185]. KGN-loaded nanoparticles have been shown to play a critical role in chondrogenesis and promote cartilage repair in vivo [131, 132, 186].

Nanoparticles-based gene delivery has been explored for promoting chondrogenesis (Fig. 7g). Nanoparticles delivered plasmid encoding $T G F-\beta 1$ can increase $T G F-\beta 1$ expression 
in chondrocytes and therefore promote the proliferation [84]. PLGA nanoparticles encoding TGF- $\beta 1$ can be transfected to seed cells such as adipose-derived stem cells to upregulate expression of chondrogenesis-related genes such as COL2a1, SOX9, and ACAN [133]. SOX9 is an essential transcription factor for the chondrogenic differentiation which is crucial for COL II and ACAN expression [187]. SOX9 genes in combination with PLGA nanoparticles increase the transfection efficiency into human mesenchymal stem cells for chondrogenesis [71].

\subsection{Perspective of Novel Therapeutic Schemes}

\subsubsection{Targeting Synovial Membrane and Subchondral Bone}

Synovial membrane inflammation increases production of the proteolytic enzymes and ROS, which aggravates matrix degradation and contributes to OA progression [6]; therefore, it has been recognized as a diagnosis and/or therapy target [188]. Macrophage-associated inflammatory infiltrate can be found in OA cartilage, and the ablation of macrophages is beneficial for cartilage health and joint integrity [189]. Active targeting can be used for labeling activated macrophages as the diagnosis of OA cartilage (Fig. 8a). For example, folate receptor (FR) is expressed by activated macrophages in the inflammatory environment. Nanoparticles conjugated with near-infrared dye and folic acid (FA) can be used as probes to detect activated macrophages, quantify severity and deliver drugs $[190,191]$. Another targeting strategy is using nanoparticles with the conjugation of certain polysaccharides of glucose such as dextran which can be selectively internalized by macrophage cells due to their expression of dextran-binding C-type lectins and scavenger receptors [192]. The major principles for the nanoparticlesbased therapy targeting synovial membrane include macrophage depletion or re-education [193]. PEGylated Ag nanoparticles decorated with folic acid (FA) can target inflamed synovial membrane to induce M1 macrophage apoptosis and M2 macrophage polarization [191]. In addition, it is also possible to decrease macrophage recruitment by inhibiting of $\mathrm{C}-\mathrm{C}$ Motif Chemokine Ligand 2 (CCL2)-C-C chemokine receptor type 2 (CCR2) signaling pathway or inhibit macrophage survival by inhibiting colony-stimulating factor 1 receptor (CSF1R) signaling [194]. Notably, cell membrane-camouflaged nanoparticles, prepared by the fusion of cellar membrane with nanoparticles, exhibit great potential in elongating circulation time, evading immune responses, and effective targeting on specific tissues or cells [195]. For example, macrophage-derived microvesiclecoated nanoparticles, which mimic macrophages, can targeting Macrophage-1(Mac-1) and CD44 to contribute to the drug delivery [196]. In addition, coating nanoparticles with neutrophil membrane are an ideal decoy of neutrophil-targeted biological molecules. These nanoparticles neutralize proinflammatory factors and inhibit synovial inflammation [197].

Dysregulated subchondral bone remodeling in OA leads to bone attrition and sclerosis, subchondral plate thickens, and osteopenic subchondral cancellous bone [198-201]. Hence, drug delivery to recover subchondral bone function is also needed (Fig. 8a). However, there is still a lack of acknowledged treatment based on the regulation of subchondral bone function. Before intervention for subchondral bone reconstruction disorders, much work needs to be done to clarify the pathogenesis of subchondral bone disorders, the regulatory relationship with cartilage, effective targeted drugs and the time window for intervention.

\subsubsection{Pain Relief Targeting Nervous System}

Inflammatory pain in $\mathrm{OA}$ is controlled by both immune system and nociceptive neurons [147]. Inflammatory pain signals produced by pro-inflammatory cytokines and chemokines stimulations (e.g., TNF, IL-1, granulocyte-macrophage colony-stimulating factor (GM-CSF), NGF and prostaglandin E2 (PGE2)) can be detected by somatosensory neurons (nociceptors) [147]. As the cell bodies of somatosensory neurons are clustered in dorsal root ganglia (DRG), signals are firstly carried to the dorsal horn of the spinal cord via dorsal root ganglia then transmitted to the brain via central axonal terminals [147]. Multiple tissues surrounding cartilage (including the synovium, ligament, osteochondral junction, and meniscus) are densely innervated [202-205], which may be the new therapy targets for pain relief (Fig. 8b).

Neurotrophins includes NGF, brain-derived neurotrophic factor (BDNF), neurotrophin 3 (NT3) and neurotrophin 4 (NT4) are important in afferent sensitization of nociceptors in OA [147, 206-208]. Since NGF is highly expressed in 


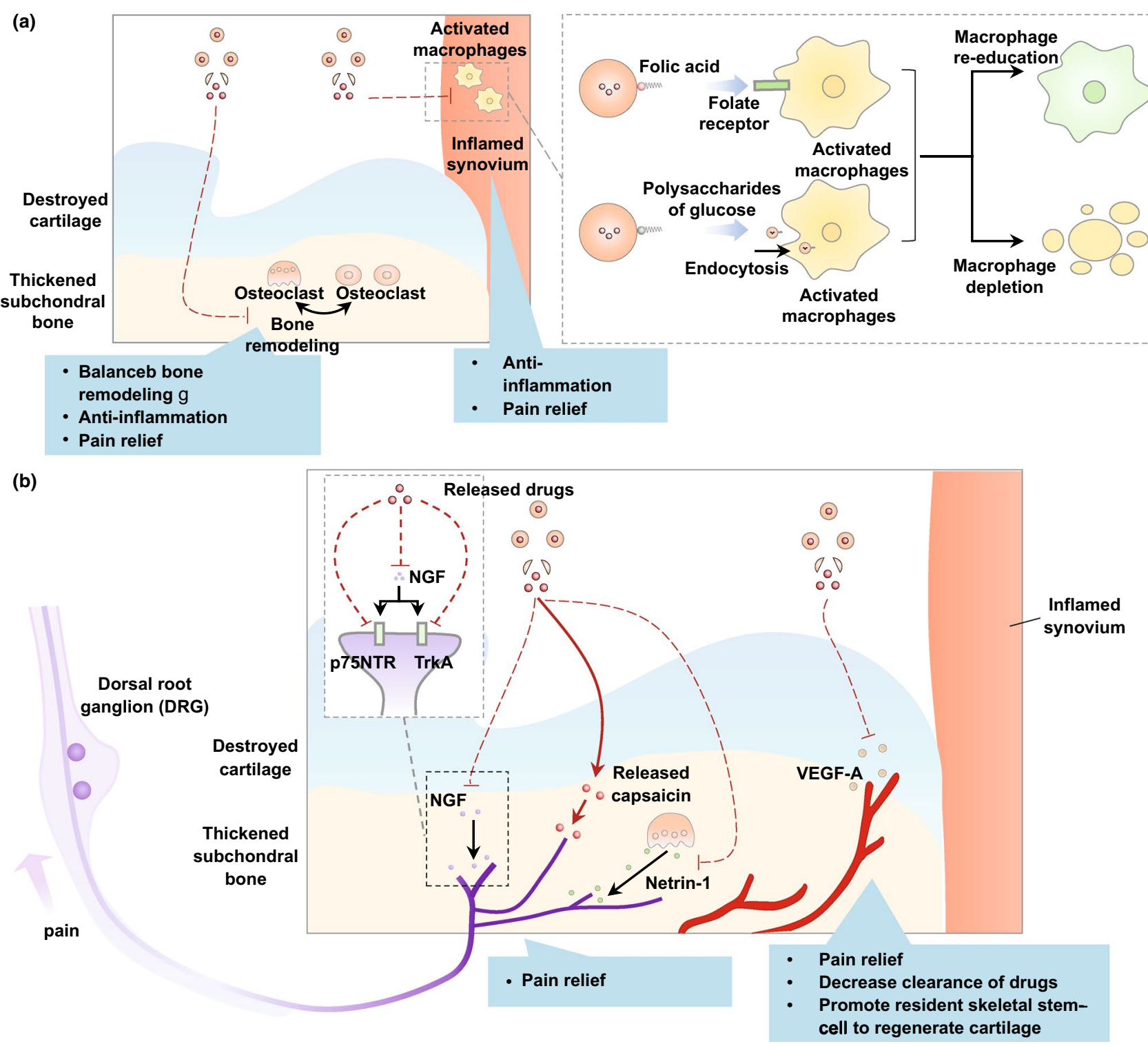

Fig. 8 Other potential target tissues in addition to cartilage according to the known pathological mechanisms. a Schematic graph illustrates nanoparticles-based therapy targeting synovial membrane and subchondral bone. b Schematic graph illustrates nanoparticles-based therapy targeting nerves and blood vessels. Abbreviations: VEGF-A, vascular endothelial growth factor A; NGF, nerve growth factor; TrkA, tropomyosinreceptor-kinase A; p75NTRs, p75 neurotrophin receptors

the inflamed synovium and at the osteochondral junction for afferent sensitization of nociceptors, underlying pain management strategy is to inhibit NGF signal transduction by using the related inhibitor, NGF-neutralizing monoclonal antibodies and/or siRNA encapsulated nanoparticles [207, 208]. The blocking of NGF receptor including tropomyosinreceptor-kinase $\mathrm{A}$ (TrkA) and $\mathrm{p} 75$ neurotrophin receptors (p75NTRs) inhibits nociceptor sensitization by NGF [209]. In addition, capsaicin derived from chili peppers induces excitation and subsequent desensitization, which is also a delivered candidate for pain relive [210].

Sensory innervation in OA can be mediated by osteoclast-initiated subchondral bone remodeling [202] Netrin-1 secreted by osteoclasts induce sensory innervation and OA pain through its receptor Deleted in Colorectal Cancer (DCC) [202]. Possible pain relief strategy including osteoclast inhibition (e.g., gene delivery by nanoparticles for inhibition RANKL production) or 
netrin-1 production inhibition (e.g., gene delivery by nanoparticles for inhibition netrin-1) is emerging.

\subsubsection{Pain Relief Targeting Blood Vessel}

Angiogenesis is another factor that contribute to structural damage and OA pain $[211,212]$. Increased vascularization in OA is mainly caused by inflammationassociated macrophage infiltration and reduced cartilage resistance to angiogenesis [211]. As some pathways and molecules stimulate both vascular cells and nerve growth, sensory nerves often grow along new blood vessels which disrupt the osteochondral junction and penetrate non-calcified articular cartilage [211, 212]. Consequently, both vascularization and inflammation contribute to nerves sensitization and increased pain $[211,212]$. In addition, angiogenesis in OA cartilage increased clearance of drugs. Moreover, anti-angiogenesis is capable of promoting resident skeletal stem-cell to regenerate cartilage [213]. Therefore, antiangiogenic nanoparticles to relieve pain and slow the progression of joint damage is a potential strategy for OA therapy (Fig. 8b).

Anti-angiogenesis can be divided into direct inhibition (e.g., targeting vascular cells) or indirect inhibition (e.g., reducing inflammation, inhibiting the matrix degradation and osteochondral channel formation). Nanoparticles for anti-angiogenesis in OA has not been reported previously. Anti-angiogenesis nanoparticles in tumor therapy also have potential for the treatment of OA since the neovascularization mechanisms are similar. Several growth factors (e.g., vascular endothelial growth factor A (VEGF-A), bFGF and TNF- $\alpha$ ) have been proved to stimulate quiescent vascular endothelium to enter the cell cycle [214], which are possible to be the main targets for nanoparticles anti-angiogenesis therapy (Fig. 5b). For example, Au nanoparticles bind to heparin-binding growth factors (HB-GFs) such as VEGF165 and bFGF to inhibit angiogenesis [215]. Ag nanoparticles show anti-angiogenic activity by downregulating PI3 K/Akt pathway and inhibiting HIF- $1 \alpha$ protein accumulation to further inhibit the expression of VEGF-A [216, 217]. Nanoparticles-based gene delivery to inhibit expression of genes such as VEGF and HIF- $1 \alpha$ is also promising for anti-angiogenesis therapy [218].

\subsection{Personalized Therapy According to the Pathology Stages}

The clinical heterogeneity of OA affects the therapeutic outcome, as different phenotypes need specific therapeutics. Therefore, the design of "smart" nanoparticles with diverse physicochemical properties for personalized therapies needs to be based on different etiological factor and pathological mechanisms. For example, in senile OA, disease progression is associated with increased chronic inflammation and mitochondrial dysfunction [219]. In contrast, in post-traumatic OA, a vigorous inflammatory response occurs very early after joint injury and then sustained at a lower level [220]. The determination of the optimal approach and timing of anti-inflammatory interventions will provide reference for the control release of nanoparticles. Meanwhile, manipulating one aspect of mechanism may affect another. For example, inhibiting inflammation at optimal stage may also indirectly promote chondrogenesis [221]. At the later stage of OA with severe cartilage defects, anti-inflammatory therapy may only relieve the symptoms. Modifications of nanoparticles to enhance function of cartilage protection and/or promotion of chondrogenesis are needed.

The convergence of biomaterial science and biomedicine opens unprecedented opportunities for the diverse medical applications of nanoparticles. Thus, in the future, with the development of noninvasive diagnostic technology, it is worthwhile to identify the pathological characteristics of each patient's joint before modification of size, charge, and surface-functional properties of nanoparticles for individualized treatment.

\section{Perspective of "Smart" Bioresponsive and Multi-modality Nanoparticles}

\subsection{Bioresponsive Nanoparticles for Controlled Delivery}

Tradition drug delivery system provides sustainable drug release; however, it is likely to cause sub- or supra-therapeutic drug levels locally since the disease activity changes over time. Ideal "smart" nanoparticles ensure that the drug will be released with proper rates at the target sites, and 
therefore minimize non-specific toxicity and enhance the therapy efficacy. The controlled release, depending on the biological signals (e.g., pain intensity) or pathological abnormalities (e.g., severity degree) of OA, can be achieved by responding to endogenous and/ or exogenous stimulus. The release of external-responsive nanoparticles can be subjectively controlled (e.g., when pain occurs) by a physician or patient, whereas the release of internal stimuli-responsive nanoparticles can be triggered depending on objective pathological changes.

Making full use of the nanomechanical properties is beneficial to regulate the smart intra-articular drug delivery. The basal drug release rate and trigger energy should be fully balanced during the design and fabrication. Nano-motors as a research hotspot have been regarded as the new generation of drug delivery system owing to the tiny size and unique mobility [222]. External-responsive nanoparticles, precisely powered by external field, such as magnetic field, electric field, and ultrasonic field, can be developed as nanomotors to penetrate cartilage tissue and trigger the drug release [222, 223]. Joint movement directly affects the retention and penetration of nanoparticles, and at the same time, the heat generated by friction also indirectly affects the degradation of nanoparticles. The design of mechanical stimuli or thermal responsive nanoparticles could be inspired by the joint movement. This section highlights the development opportunities of stimulus-responsive nanoparticles for interarticular drug delivery.

\subsubsection{External-Responsive Nanoparticles}

Magnetically guided nanoparticles including iron oxide nanoparticles, iron oxide hybrid nanoparticles, and other magnetic nanoparticles may be applied for the controlled delivery by a magnetic guidance under a permanent magnetic field or alternating magnetic field [223, 224]. An extracorporeal magnetic field near cartilage can be applied for magnetic guidance and on-demand release after the intraarticular injection of a magnetically responsive nanoparticles (Fig. 9a). For instance, Jafari et al. utilized dynamic magnetic fields to guide the transport of magnetic nanoparticles through the entire thickness of bovine articular cartilage [225]. The alternating magnetic field induces a nearly 50 times increase in magnetic nanoparticles transport as compared with static field conditions [225]. Moreover, magnetic nanoparticles can be used as cell labels and guided seeding for cartilage repair [226]. Meanwhile, the magnetic nanoparticles (e.g., $\mathrm{Fe}_{3} \mathrm{O}_{4}$ )-mediated physical stimuli promote chondrogenic differentiation in vitro by enhance level of sulfated glycosaminoglycan (sGAG) and collagen synthesis [227]. It should be noted that whether the increased temperature under the alternating magnetic field has adverse effect on the cartilage repair is unclear and should be confirmed before the therapy application. Ultrasound waves can trigger controlled release of drugs through the thermal and/or mechanical effects (Fig. 9b) [224]. Cartilage penetration depth can be regulated through cavitation phenomena or radiation forces [224]. Since pulsed ultrasound has been proved to be effective in pain relief in OA, therapy based on ultrasound responsive nanoparticles may lead to both controlled drug release and function improvement [228, 229]. Photosensitiveness-induced structural modifications of the nanoparticles can trigger drug release in response to light (Fig. 9c) [224]. The irradiation wavelength and power can be adjusted to achieve noninvasive controlled release by cleaving the light-sensitive chemical bonds or assembling in response to light [230-232]. Photoresponsive nanoparticles, which achieve drug release in response to ultraviolet, visible or near-infrared (NIR) regions, can be engineered. However, due to the strong scattering properties of soft tissues, the penetration depth of visible light and ultraviolet light is limited to less than $10 \mathrm{~mm}$, so they are not suitable for intra-articular delivery [224]. NIR laser (700-1,000 nm range) can replace the ultraviolet-visible light with a deeper tissue penetration $(2-5 \mathrm{~cm})$ [224]. For example, doxorubicin-loaded hollow gold nanoparticles convert the photon energy adsorbed during irradiation $(808 \mathrm{~nm})$ into heat to trigger drug release [233].

\subsubsection{Internal Stimuli-Responsive Nanoparticles}

OA (especially in knee), as a joint failure with myriad causes, is affected more by mechanical stress rather than systemic factor such as inflammation, aberrant metabolic regulation, and obesity [234]. The fabrication of mechanical stimuli-responsive nanoparticles has been reported previously [235, 236]. For example, Holme et al. fabricated lenticular liposomes made from an artificial 1,3-diaminophospholipid which are stable under static conditions but release their encapsulated contents at elevated shear stress 
(a) Magnetically guided nanoparticles
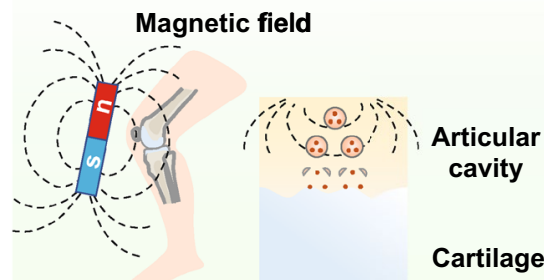

(d) Mechanosensitive nanoparticles

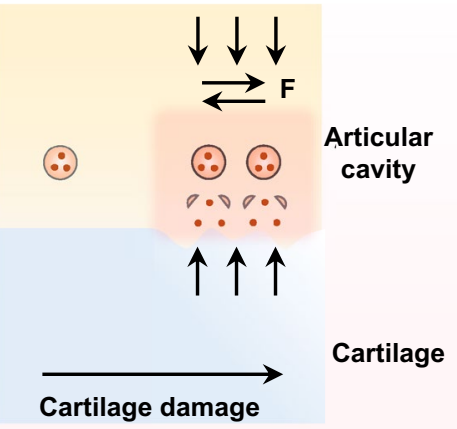

(b) Ultrasound responsive nanoparticles

(c) Light-sensitive nanoparticles
Ultrasound

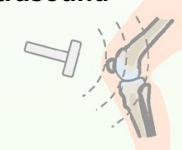

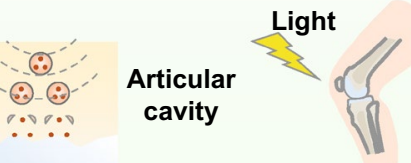

Cartilage

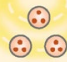

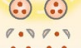

Articular cavity

Cartilage

(e) Thermal responsive nanoparticless

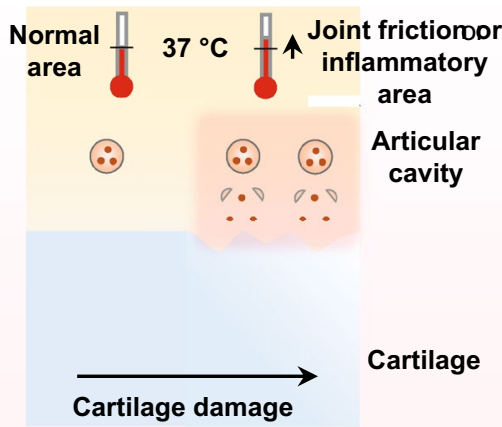

(f) $\mathrm{pH}$-responsive nanoparticles

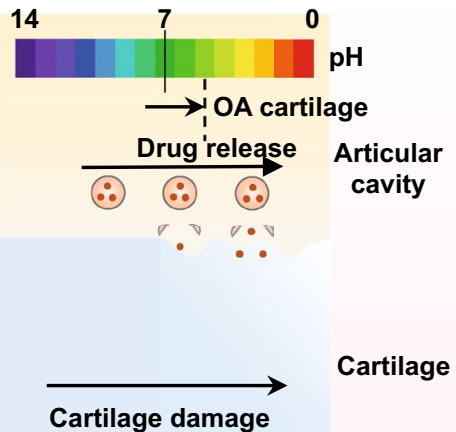

(g) Enzyme-responsive nanoparticles

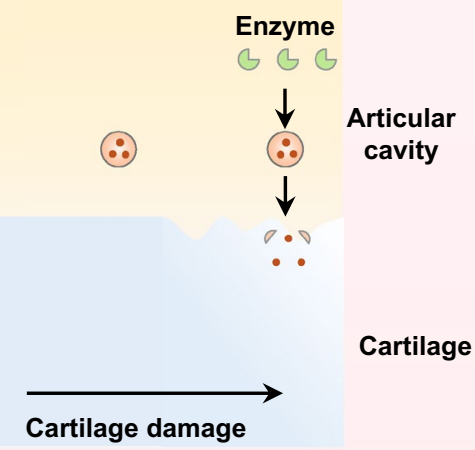

(h) Hypoxia-sensitive nanoparticles

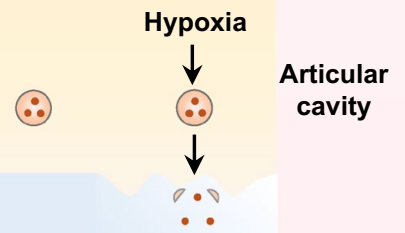

(i) Composition/Structure of HM

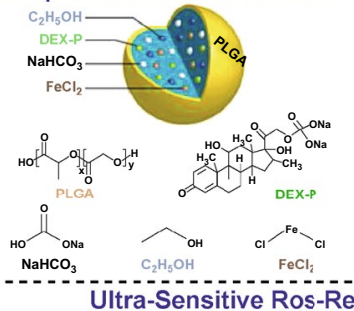

Ultra-Sensitive Ros-Responsive Drug Release

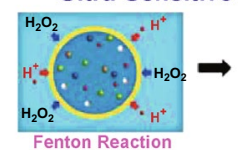

Reduce Local pH $\mathrm{C}_{2} \mathrm{H}_{5} \mathrm{OH}+\mathrm{H}_{2} \mathrm{O}_{2} \stackrel{\mathrm{Fe}^{2}}{\rightarrow} \mathrm{CH}_{3} \mathrm{COOH}+\mathrm{H}_{2} \mathrm{O}$ $\mathrm{CH}_{3} \mathrm{COOH} \rightarrow \mathrm{CH}_{3} \mathrm{COO}^{-}+\mathrm{H}^{+}$
Treatment of Osteoarthritis<smiles>C1=C[C@H]2C=C[C@H]1C2</smiles>

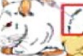
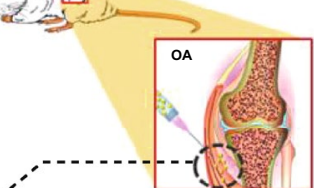

SBC Decomposition
Generate $\mathrm{CO}_{2}$ Bubbles to Disrupt HM $\mathrm{HCO}_{3}^{-}+\mathrm{H}^{+} \rightarrow \mathrm{H}_{2} \mathrm{CO}_{3}$ $\mathrm{H}_{2} \mathrm{CO}_{3} \rightarrow \mathrm{H}_{2} \mathrm{O}+\mathrm{CO}_{2}$
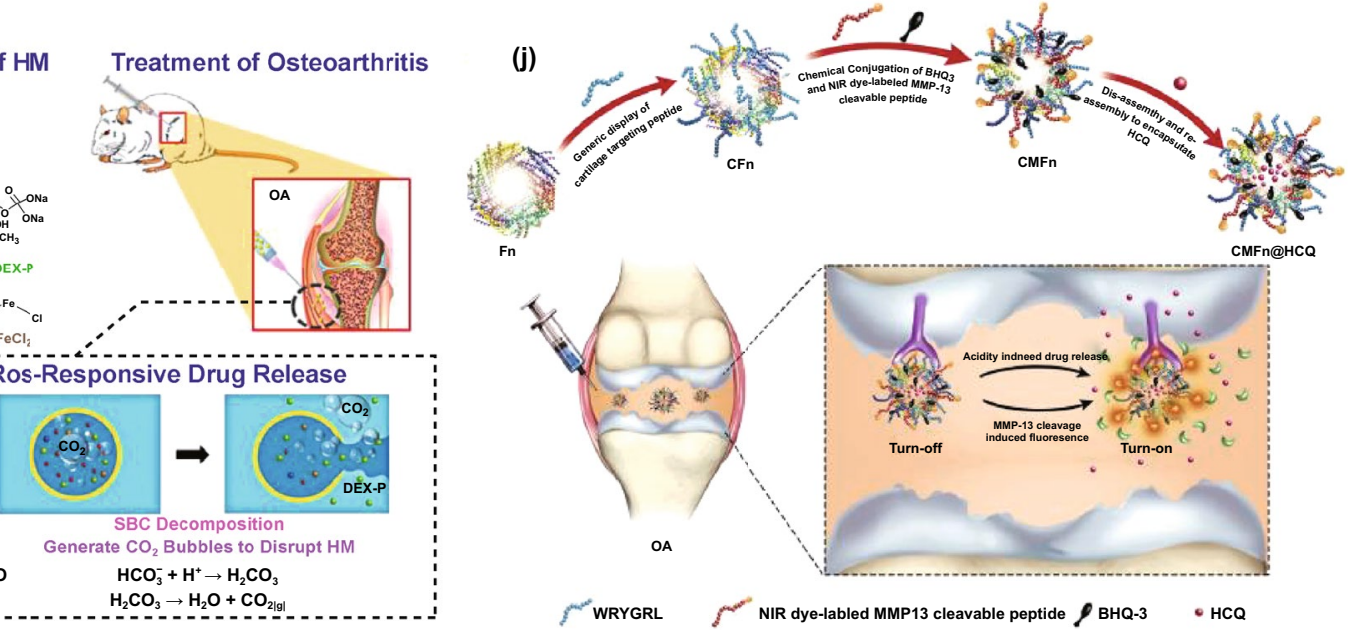
4Fig. 9 Potential therapeutic strategies by using stimuli-responsive nanoparticles for control delivery in OA. a-c Schematic graphs illustrate external-responsive nanoparticles for OA therapy. d-h Schematic graphs illustrate internal stimuli-responsive nanoparticles for OA therapy. Reproduced with permission [242]. Copyright (C) 2015 American Chemical Society. i Example of $\mathrm{pH}$ responsive nanoparticles for OA imaging and therapy. $\mathbf{j}$ Example of enzyme responsive nanoparticles for OA therapy. Reproduced with permission [53]. Copyright (c) 2019 Elsevier Ltd. Abbreviations: OA, osteoarthritis Copyright (C) 2015 American Chemical Society. i Example of pH responsive nanoparticles for OA imaging and therapy. $\mathbf{j}$ Example of enzyme responsive nanoparticles for OA therapy. Reproduced with permission [53]. Copyright (C) 2019 Elsevier Ltd. Abbreviations: OA, osteoarthritis

[235]. It is theoretically possible to use such nanoparticles on the wear and tear surface to achieve automatic release of drugs for improved treatment outcome (Fig. 9d). The general concept is based on the assembly and disassembly of mechano-responsive structures under the changing mechanical environment.

Thermal responsive hollow nanoparticles have been reported to enhance the delivery efficiency in OA (Fig. 9e) $[155,237]$. Further functional improvements of thermal responsive nanoparticles can be learned from experience in cancer research [238]. Generally, thermal responsive nanoparticles in cancer therapy remain stable at body temperature $\left(\sim 37^{\circ} \mathrm{C}\right)$ and release drug rapidly in a higher temperature $\left(\sim 40-42{ }^{\circ} \mathrm{C}\right)[223,224]$. Potential of using such nanoparticles in OA therapy is based on the two possible reasons. Firstly, the joint temperatures in OA (especially in the defected area) may be higher which is related to greater joint friction effects and retarded cooling [239]. Secondly, joint inflammation may also lead to high local temperature.

Lower $\mathrm{pH}$ during cartilage degeneration alters the proteoglycans structure and concentration, leading to decreased viscosity [240]. $\mathrm{pH}$-responsive nanoparticles may tune drug release and cell uptake in OA cartilage for achieving high therapeutic performance (Fig. 9f). For instance, chitosan as a $\mathrm{pH}$ responsive polysaccharide has been utilized to fabricate $\mathrm{pH}$-responsive nanoparticles which can release faster in acid environment ( $\mathrm{pH} \sim 5.5)$ than neutral environment [241]. In a sodium bicarbonate-encapsulated particle, the release of anti-inflammatory drug can also be triggered by an acidic milieu induced decomposition reaction (Fig. 9i) [242]. Lysosomal molecules are upregulated in OA which involved in the modulation of chondrocytes death [243]. The acidic luminal $\mathrm{pH}$ in $\mathrm{OA}$ lysosome compartment is also likely to trigger the release of $\mathrm{pH}$ sensitive particles in defected cell for effective intracellular drug accumulation. The feasibility still needs to be verified as $\mathrm{pH}$-sensitive nanoparticles may lead to leakage of lysosomal enzymes by disturbing lysosomal membranes and further cause autophagy and cell death.

Since matrix-degrading enzymes, such as MMP3, MMP13, and ADAMTS5, are upregulated in OA cartilage, they can be exploited to achieve enzyme-mediated drug release (Fig. 9g). For example, nanoparticles containing matrix metalloproteinase substrate peptide (e.g., H2N-GPLGVRGC-SH as MMP-13 cleavable specific peptide substrate) undergo morphological transition for drug release when react with matrix metalloproteinase in a mouse OA model (Fig. 9j) [53].

As an avascular tissue, cartilage is hypoxic in nature. This property can be utilized to design hypoxia-sensitive nanoparticles for the controlled release of drug in cartilage. Hypoxia-sensitive nanoparticles are normally constructed with hypoxia-sensitive materials or derivatives such as 2-nitroimidazole, nitroimidazole, metronidazole, azobenzene, nitro-benzene derivatives and iridium (III) complexes, etc. [223]. Before the design, oxygen tension in health cartilage and OA cartilage needs to be confirm.

\subsection{Multi-modality Nanoparticles}

\subsubsection{Nanoparticles in OA Diagnosis}

Cartilage degradations in OA can only be diagnosed when the size of defected area is larger enough. Consequently, prompt diagnosis is often important for early treatment, however difficult due to the lack of diagnostic options. To fill this gap, there is a significant growth of interest in the use of nanoparticles as optical imaging probes in diagnostics (Fig. 10a, c, d). Deeper imaging penetration, photostability, and biocompatibility should be fully considered.

Noble metal nanoparticles (e.g., gold nanoparticles), fluorescent nanoparticles, and magnetic nanoparticles, with advantages of increased the sensitivity, better detection capabilities and ease of operation have been studied for possible application of OA diagnosis [54, 186, 244-248]. The advantages of fluorescent dyes include high selectivity, high sensitivity, and high fluorescence quantum yield; however, obstacles including potential toxicity, poor fluorescence stability limit their medical use. Metal oxide nanoparticles can be used as contrast agents in magnetic resonance imaging. 
(a)

\section{0}

Noble metal nanoparticles (e.g. gold nanoparticles), fluorescent nanoparticles, and magnetic nanoparticles
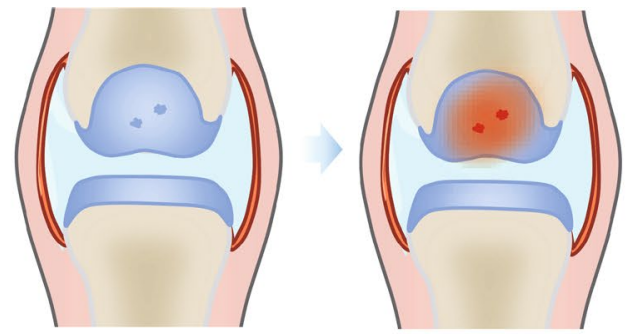

OA
Fluorescence

imaging, MRI,

PET/SPECT, $X$ -

ray/CT, multimodal

imaging

\section{(b)}

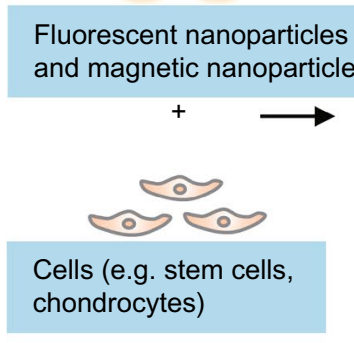

(c)
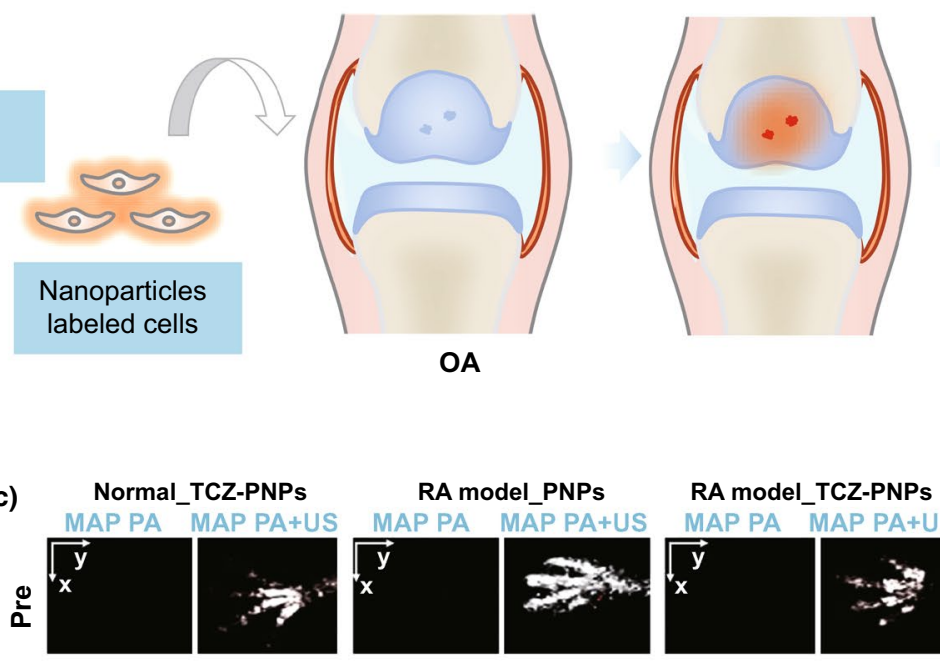

Fluorescence

imaging, MRI,

PET/SPECT, $X$ -

ray/CT, multimodal

imaging

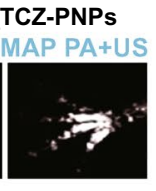

RA model_PNPs

RA model_TCZ-PNPs
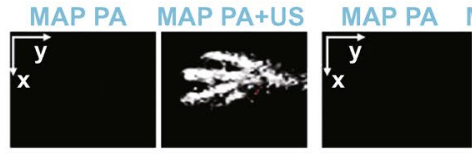
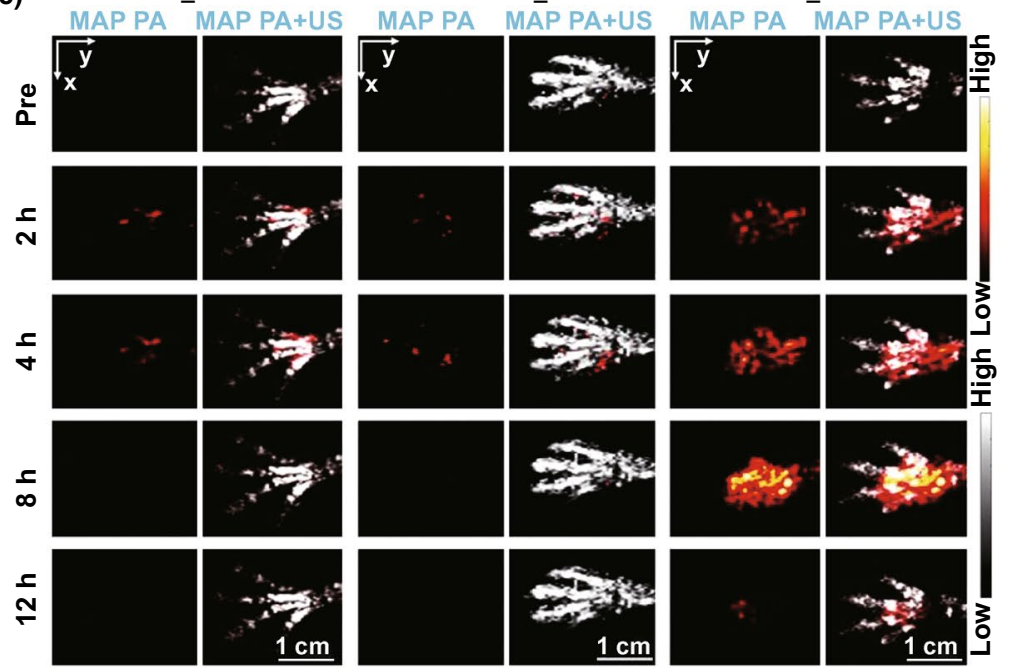

(d)

(d) Transverse
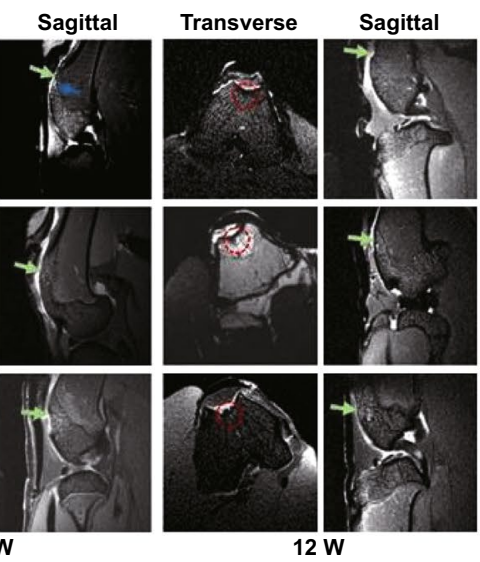

Group C

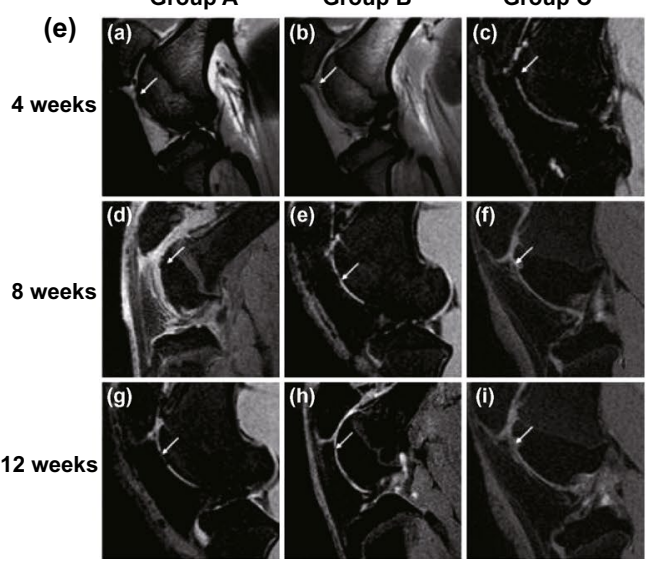


\Fig. 10 Multiples applications of nanoparticles in OA. a Schematic graph illustrates application of nanoparticles in OA diagnosis. b Schematic graphs illustrate application of nanoparticles in cell tracking. c Example of fluorescent labeled nanoparticles in cartilage diseases. Reproduced with permission [252]. Copyright (C) 2020 Wiley$\mathrm{VCH} \mathrm{GmbH}$. d Example of magnetic nanoparticles in OA diagnosis. Reproduced with permission [186]. Copyright 2020, Ivyspring International Publisher. e Example of magnetic nanoparticles in cell tracking. Reproduced with permission [260]. Copyright 2012, Springer Nature. Abbreviations: CT, computed tomography; MRI, magnetic resonance imaging; OA, osteoarthritis; PET, positron emission tomography; SPECT, single-photon emission computed tomography

For diagnosis, metal nanoparticles are more stable than organic fluorescent labels and the surface can be modified (e.g., by polymers and surfactants). Gd and Mn elements are used as a T1-weighted contrast agent for magnetic resonance (MR) imaging [249, 250]. However, drawbacks such as toxicity and tissue accumulation limit the applications [251]. Fe element-based nanoparticles with superparamagnetism have been studied as nanoprobe to provide dark T2-weighted imaging [186, 244-246]. For example, synthesized magnetite $\mathrm{Fe}_{3} \mathrm{O}_{4}$ nanoparticles penetrate cartilage and the size of impregnated particles shows the degree of cartilage degradation [245].

Gold nanoparticles-based probes are appealing for the diagnosis of OA. They are inert and therefore are biocompatible and non-toxic. The surfaces of gold nanoparticles can be easily modified. Moreover, gold nanoparticles can be incorporated into larger structures such as polymers or liposomes for enhanced diagnostic applications [247]. For example, by conjugating gold nanoparticles with a FITCmodified ADAMTS-4- specific peptide (DVQEFRGVTAVIR), the gold nanoparticles probe has been developed for detection of aggrecanase [248]. When the probe is incubated with aggrecanase ADAMTS-4, the fluorescence intensity significantly increases [248]. As a result, the increased aggrecanase activity can be detected for early diagnosis of cartilage-degradation [248].

NIR-II photoacoustic molecular imaging is emerging as a strategy for effective diagnosis. Compared to conventional optical imaging modalities, NIR-II photoacoustic molecular imaging is generated by the absorption of light by a molecule under short pulse laser irradiation, which overcomes the common optical diffusion limit [252-254]. NIR-II-conjugated polymer nanoprobe displays effective noninvasive diagnosis of cartilage disease [252].

Nanoparticles probes can also be designed based on the changes of inflammation states during OA. As the macrophage activity often increases in OA, a nanoprobe has been fabricated by conjugating hyaluronic acid with nearinfrared dye and folic acid (FA), to bind to folate receptors of macrophages [190]. In addition, the overproduction of nitric oxide (NO) in OA stimulates the production of proinflammatory cytokines, such as TNF $\alpha$ and IL-1 $\beta$ [255]. By encapsulating the NO sensing molecules (e.g., 4-amino-5methylamino-2', 7'-difluorofluorescein DiaminofluoresceinFM (DAF-FM)) within nanoparticles, NO release can be monitored which permits the indirect real-time evaluation of OA development [255].

\subsubsection{Nanoparticles in Cell Tracking}

Advances in cell therapy have emphasized the role of chondrocytes and mesenchymal stem cells (MSCs) with chondrogenic differentiation ability, migratory, anti-inflammatory and/or immunosuppressive properties in treating cartilage lesions (Fig. 10b, e) [256]. A noninvasive means of imaging and tracking the cell fate after in vivo implantation could be extremely valuable. Quantum dots such as fluorescencelabeled mesoporous silica nanoparticles and superparamagnetic iron oxide (SPIO) nanoparticles have been developed for in vitro and in vivo bioimaging [257, 258]. The basic principle for the cell-labeling agents is that enough number of nanoparticles should bind to cells to be detectable. Besides, nanoparticles should not interfere the cellular functions such as cell viability and differentiation capacity. By using superparamagnetic iron oxide nanoparticles (SPIONs) cell labeling, magnetic resonance imaging (MRI) can visually monitor the in vivo dynamic biodistribution of implanted cell. With good biocompatibility, SPIONs do not affect cell viability, proliferation, and differentiation capabilities [259, 260].

\subsubsection{Theranostic Applications of Metal Nanoparticles}

Theranostics are combinatorial approaches that aim to deliver therapy and examine the effect at the same time. Combining the nanoparticle-based drug delivery strategy with the diagnostic techniques of nanoparticles provides the possibilities to achieve this aim [261]. For example, chitosan-modified $\mathrm{Fe}_{3} \mathrm{O}_{4}$ nanoparticles have been fabricated for delivery KGN, which can be used as for both diagnosis under magnetic resonance (MR) imaging and osteochondral regeneration [186]. Noble 
metal nanoparticles, such as gold, silver, or a combination of both, with advantages of high surface-to-volume ratio, ease of synthesis and highly tunable optical properties, can comprise nucleic acids (DNA/RNA), drugs and biocompatible polymers (e.g., polyethylene glycol and PEG) for delivery. In addition, there unique properties can be utilized (e.g., gold nanoparticles for diagnostics [262]; silver and platinum (Pt) nanoparticles as reactive oxygen species scavengers [263]). For example, silver nanoparticles have been explored to alleviating joint inflammation via reeducate macrophages [191]. It is possible to expect more effective therapy through the diagnosis and prediction of pathological process to control of drug release by adding stimuli-responsive components in nanoparticles. However, the major shortcoming is that noble metal NPs are difficult to be degraded from organisms which may interfere medical diagnoses. Moreover, the degradation by lysosomes within cells may produce toxic free metal ions such as $\mathrm{Ag}^{+}$and $\mathrm{Au}^{+/ 3+}$, which affect cell homeostasis [264]. As such, it is important to balance action, clearance and toxicity. Zinc (Zn), and magnesium $(\mathrm{Mg})$, as the best-explored biodegradable metals for orthopedic applications, hold promise to be fabricated as the moiety of nanoparticles to solve the non-degradable problems [265, 266]. After degradation, the cations (e.g., $\mathrm{Zn}^{2+}, \mathrm{Mg}^{2+}$ ) may be resorbed by the negative charged cartilage matrix. However, $\mathrm{Zn}^{2+}$ in OA increases the synthesis and secretion of matrixdegrading enzymes which aggravated the progression of OA [267]. In contrast, Mg nanoparticles may be more promising for OA therapy as intra-articular injections of $\mathrm{Mg}^{2+}$ at $0.5 \mathrm{~mol}$ $\mathrm{L}^{-1}$ attenuate the progression of OA by inhibiting inflammation and matrix-degrading enzymes [268]. Moreover, particles containing magnesium powder can continuously evolve gaseous $\mathrm{H}_{2}$ which can also effectively mitigate joint inflammation [269]. Future smart design of theranostic nanomaterials needs to improve the stability of biomaterials and reduce toxicity for co-delivery of multiple components and more sensitive detection [270]. At the same time, how to design the bio-inspired materials for better interaction with the pathological features needs to be explored [271, 272].

\section{Translation from 'Bench to Bedside'}

Dozens of nanoparticles including liposome, polymer, micelle, inorganic, nanocrystal and protein nanoparticles have received FDA approval and are currently available for clinical use for the therapy of disease such as kidney disease, cancer, sclerosis, and bone defect [273, 274]. Preclinical studies discussed above show the potential of nanoparticles to improve the drug delivery efficiency for the treatment of OA. However, to date, there is still limited clinical application for the therapy of OA. For example, FX006 as a PLGA-based drug delivery system for the treatment of OA in clinical trials results in clinically minimal improvement in pain relief [275]. To bridge this gap, there is a need to understand the challenges. Firstly, the basic principle for the clinical application is low toxicity. Toxicological studies on the application of nanoparticles in cartilage are still few. Besides, more efforts are needed to confirm the pharmaceutical stability and the in vivo behavior, such as cellular and molecular interactions. It is apparently a great challenge to choose effective pharmacological agents, given that the clinical efficacy and mechanisms (e.g., hyaluronic acid, glucosamine, and chondroitin) for OA therapy are still uncertain. More importantly, the modification of nanomaterials in basic research makes their structures and components more complex and their functions more diverse, which need high development cost for the clinical translation. In contrast, it is more feasible to evaluate the clinical safety and efficacy of nanomaterials with simpler structures and components. However, maybe it is "Easier Said Than Done." For the avascular cartilage tissue, it is more difficult to ensure the efficacy with simple materials. Extensive efforts are needed for controlling the complexity without decreasing therapeutic efficacy.

\section{Conclusions}

Current nanoparticles-based intra-articular delivery represents a new frontier arisen from an urgent need to address the issues of low drug retention. Ideal nanoparticles with suitable size, charge, and modification are supposed to penetrate the cartilage matrix easily and provide sustainable drug or gene delivery upon demand, which is conducive to the relief of OA symptom and cartilage regeneration. A comprehensive understanding of nanoparticle transport and nano-cartilage interactions in vivo is vital for improving therapeutic efficacy, avoiding or minimizing the adverse effects. Further advances are in progress to bring forth more "smart" diagnosis and therapy with the constant improvement of material technology and continuous evolvement of the understanding of OA. We anticipate that with better and 
fundamental understanding on cartilage-nanoparticle crosstalk, multidisciplinary collaborations, we will definitively be able to advance nanomedicine toward early diagnosis and effective therapy for OA.

Acknowledgements The work was supported by RGC Themebased Research Scheme of Hong Kong (T13-402/17N), National Natural Science Foundation of China (81802152), Natural Science Foundation of Guangdong Province (2019A1515012224), RGC Areas of Excellence (AoE/M-402/20), RGC Collaborative Research Fund (C4026-17WF), General Research Fund (14121918 and 14173917), the Innovation and Technology Commission Funding (ITS/208/18FX), and Key-Area Research and Development Program of Guangdong Province (2019B010941001).

Open Access This article is licensed under a Creative Commons Attribution 4.0 International License, which permits use, sharing, adaptation, distribution and reproduction in any medium or format, as long as you give appropriate credit to the original author(s) and the source, provide a link to the Creative Commons licence, and indicate if changes were made. The images or other third party material in this article are included in the article's Creative Commons licence, unless indicated otherwise in a credit line to the material. If material is not included in the article's Creative Commons licence and your intended use is not permitted by statutory regulation or exceeds the permitted use, you will need to obtain permission directly from the copyright holder. To view a copy of this licence, visit http://creativecommons.org/licenses/by/4.0/.

\section{References}

1. A.D. Woolf, B. Pfleger, Burden of major musculoskeletal conditions. Bull. World Health Organ. 81(9), 646-656 (2003)

2. D.J. Hunter, D. Schofield, E. Callander, The individual and socioeconomic impact of osteoarthritis. Nat. Rev. Rheumatol. 10(7), 437-441 (2014). https://doi.org/10.1038/nrrheum. 2014.44

3. I.A. Jones, R. Togashi, M.L. Wilson, N. Heckmann, C.T. Vangsness Jr., Intra-articular treatment options for knee osteoarthritis. Nat. Rev. Rheumatol. 15(2), 77-90 (2019). https://doi.org/10.1038/s41584-018-0123-4

4. K. Masuda, R.L. Sah, M.J. Hejna, E.J. Thonar, A novel twostep method for the formation of tissue-engineered cartilage by mature bovine chondrocytes: the alginate-recovered-chondrocyte (arc) method. J. Orthop. Res. 21(1), 139-148 (2003). https://doi.org/10.1016/s0736-0266(02)00109-2

5. N. Verzijl, J. DeGroot, S.R. Thorpe, R.A. Bank, J.N. Shaw et al., Effect of collagen turnover on the accumulation of advanced glycation end products. J. Biol. Chem. 275(50), 39027-39031 (2000). https://doi.org/10.1074/jbc.M0067 00200

6. A. Mobasheri, M.P. Rayman, O. Gualillo, J. Sellam, P. van der Kraan et al., The role of metabolism in the pathogenesis of osteoarthritis. Nat. Rev. Rheumatol. 13(5), 302-311 (2017). https://doi.org/10.1038/nrrheum.2017.50

7. D.R. Eyre, M.A. Weis, J.J. Wu, Articular cartilage collagen: an irreplaceable framework? Eur. Cells Mater. 12, 57-63 (2006). https://doi.org/10.22203/ecm.v012a07

8. S. Glyn-Jones, A.J. Palmer, R. Agricola, A.J. Price, T.L. Vincent et al., Osteoarthritis. Lancet 386(9991), 376-387 (2015). https://doi.org/10.1016/s0140-6736(14)60802-3

9. Y. Deng, J. Lu, W. Li, A. Wu, X. Zhang et al., Reciprocal inhibition of YAP/TAZ and NF- $\mathrm{KB}$ regulates osteoarthritic cartilage degradation. Nat. Commun. 9(1), 4564 (2018). https://doi.org/10.1038/s41467-018-07022-2

10. W. Tong, Y. Zeng, D.H.K. Chow, W. Yeung, J. Xu et al., Wnt16 attenuates osteoarthritis progression through a PCP/JNK-mTORC1-PTHrP cascade. Ann. Rheum.. Dis. 78(4), 551-561 (2019). https://doi.org/10.1136/annrh eumdis-2018-214200

11. W. Tong, Y. Geng, Y. Huang, Y. Shi, S. Xiang et al., In vivo identification and induction of articular cartilage stem cells by inhibiting NF- $\kappa \mathrm{B}$ signaling in osteoarthritis. Stem Cells 33(10), 3125-3137 (2015). https://doi.org/10.1002/stem. 2124

12. J. Martel-Pelletier, C. Boileau, J.P. Pelletier, P.J. Roughley, Cartilage in normal and osteoarthritis conditions. Best Pract. Res. Clin. Rheumatol. 22(2), 351-384 (2008). https://doi.org/ 10.1016/j.berh.2008.02.001

13. C.R. Flannery, C.B. Little, C.E. Hughes, B. Caterson, Expression of adamts homologues in articular cartilage. Biochem. Biophys. Res. Commun. 260(2), 318-322 (1999). https://doi. org/10.1006/bbrc.1999.0909

14. C.D. DiDomenico, M. Lintz, L.J. Bonassar, Molecular transport in articular cartilage-what have we learned from the past 50 years? Nat. Rev. Rheumatol. 14(7), 393-403 (2018). https://doi.org/10.1038/s41584-018-0033-5

15. D.J. Hunter, S. Bierma-Zeinstra, Osteoarthritis. Lancet 393(10182), 1745-1759 (2019). https://doi.org/10.1016/ s0140-6736(19)30417-9

16. L. Chen, F. Yao, T. Wang, G. Li, P. Chen et al., Horizontal fissuring at the osteochondral interface: a novel and unique pathological feature in patients with obesity-related osteoarthritis. Ann. Rheum. Dis. 79(6), 811-818 (2020). https://doi. org/10.1136/annrheumdis-2020-216942

17. E.M. Bouta, R.D. Bell, H. Rahimi, L. Xing, R.W. Wood et al., Targeting lymphatic function as a novel therapeutic intervention for rheumatoid arthritis. Nat. Rev. Rheumatol. 14(2), 94-106 (2018). https://doi.org/10.1038/nrrheum.2017.205

18. J.A. Block, Osteoarthritis: Oa guidelines: improving care or merely codifying practice? Nat. Rev. Rheumatol. 10(6), 324-326 (2014). https://doi.org/10.1038/nrrheum.2014.61

19. T.E. McAlindon, R.R. Bannuru, M.C. Sullivan, N.K. Arden, F. Berenbaum et al., Oarsi guidelines for the non-surgical management of knee osteoarthritis. Osteoarthritis Cartil. 22(3), 363-388 (2014). https://doi.org/10.1016/j.joca.2014. 01.003 
20. B.R. da Costa, S. Reichenbach, N. Keller, L. Nartey, S. Wandel et al., Effectiveness of non-steroidal anti-inflammatory drugs for the treatment of pain in knee and hip osteoarthritis: a network meta-analysis. Lancet 390(10090), e21-e33 (2017). https://doi.org/10.1016/s0140-6736(17)31744-0

21. A.E. Nelson, K.D. Allen, Y.M. Golightly, A.P. Goode, J.M. Jordan, A systematic review of recommendations and guidelines for the management of osteoarthritis: the chronic osteoarthritis management initiative of the U.S. Bone and joint initiative. Semin. Arthritis Rheum. 43(6), 701-712 (2014). https://doi.org/10.1016/j.semarthrit.2013.11.012

22. J. Martel-Pelletier, A.J. Barr, F.M. Cicuttini, P.G. Conaghan, C. Cooper et al., Osteoarthritis. Nat. Rev. Dis. Primers 2, 16072 (2016). https://doi.org/10.1038/nrdp.2016.72

23. K. Zhao, Y.-S. Liu, L.-Y. Nie, L.-N. Qian, N.-F. Nie et al., The influence of sample size and gender composition on the meta-analysis conclusion of platelet-rich plasma treatment for osteoarthritis. J. Orthop. Transl. 22, 34-42 (2020). https://doi.org/10.1016/j.jot.2019.10.002

24. N. Gerwin, C. Hops, A. Lucke, Intraarticular drug delivery in osteoarthritis. Adv. Drug Deliv. Rev. 58(2), 226-242 (2006). https://doi.org/10.1016/j.addr.2006.01.018

25. C. Larsen, J. Ostergaard, S.W. Larsen, H. Jensen, S. Jacobsen et al., Intra-articular depot formulation principles: role in the management of postoperative pain and arthritic disorders. J. Pharm. Sci. 97(11), 4622-4654 (2008). https:// doi.org/10.1002/jps.21346

26. A. Ghadi, S. Mahjoub, F. Tabandeh, F. Talebnia, Synthesis and optimization of chitosan nanoparticles: potential applications in nanomedicine and biomedical engineering. Caspian J. Int. Med. 5(3), 156-161 (2014)

27. S. Gelperina, K. Kisich, M.D. Iseman, L. Heifets, The potential advantages of nanoparticle drug delivery systems in chemotherapy of tuberculosis. Am. J. Respir. Crit. Care Med. 172(12), 1487-1490 (2005). https://doi.org/10.1164/ rccm.200504-613PP

28. H. Thakkar, R.K. Sharma, R.S. Murthy, Enhanced retention of celecoxib-loaded solid lipid nanoparticles after intraarticular administration. Drugs R\&D 8(5), 275-285 (2007). https://doi.org/10.2165/00126839-200708050-00002

29. M. Higaki, T. Ishihara, N. Izumo, M. Takatsu, Y. Mizushima, Treatment of experimental arthritis with poly(d, 1-lactic/glycolic acid) nanoparticles encapsulating betamethasone sodium phosphate. Ann. Rheum. Dis. 64(8), 1132-1136 (2005). https://doi.org/10.1136/ard.2004. 030759

30. C.H. Evans, V.B. Kraus, L.A. Setton, Progress in intraarticular therapy. Nat. Rev. Rheumatol. 10(1), 11-22 (2014). https://doi.org/10.1038/nrrheum.2013.159

31. J. Pradal, P. Maudens, C. Gabay, C.A. Seemayer, O. Jordan et al., Effect of particle size on the biodistribution of nanoand microparticles following intra-articular injection in mice. Int. J. Pharm. 498(1-2), 119-129 (2016). https://doi.org/10. 1016/j.ijpharm.2015.12.015

32. A.G. Bajpayee, A.J. Grodzinsky, Cartilage-targeting drug delivery: can electrostatic interactions help? Nat. Rev.
Rheumatol. 13(3), 183-193 (2017). https://doi.org/10.1038/ nrrheum.2016.210

33. B.D. Partain, M. Unni, C. Rinaldi, K.D. Allen, The clearance and biodistribution of magnetic composite nanoparticles in healthy and osteoarthritic rat knees. J. Control. Release 321, 259-271 (2020). https://doi.org/10.1016/j.jconrel.2020.01. 052

34. Y. Wei, L. Luo, T. Gui, F. Yu, L. Yan et al., Targeting cartilage egfr pathway for osteoarthritis treatment. Sci. Transl. Med. 13(576), eabb3946 (2021). https://doi.org/10.1126/scitr anslmed.abb3946

35. A.J.S. Fox, A. Bedi, S.A. Rodeo, The basic science of articular cartilage: structure, composition, and function. Sports Health 1(6), 461-468 (2009). https://doi.org/10.1177/19417 38109350438

36. B.K. Hall, S.A. Newman, Cartilage Molecular Aspects (CRC Press, 1991)

37. D.A. Rothenfluh, H. Bermudez, C.P. O'Neil, J.A. Hubbell, Biofunctional polymer nanoparticles for intra-articular targeting and retention in cartilage. Nat. Mater. 7(3), 248-254 (2008). https://doi.org/10.1038/nmat2116

38. P.A. Torzilli, J.M. Arduino, J.D. Gregory, M. Bansal, Effect of proteoglycan removal on solute mobility in articular cartilage. J. Biomech. 30(9), 895-902 (1997). https://doi.org/ 10.1016/s0021-9290(97)00059-6

39. K.A. Elsaid, L. Ferreira, T. Truong, A. Liang, J. Machan et al., Pharmaceutical nanocarrier association with chondrocytes and cartilage explants: influence of surface modification and extracellular matrix depletion. Osteoarthritis Cartil. 21(2), 377-384 (2013). https://doi.org/10.1016/j. joca.2012.11.011

40. B.C. Geiger, S. Wang, R.F. Padera Jr., A.J. Grodzinsky, P.T. Hammond, Cartilage-penetrating nanocarriers improve delivery and efficacy of growth factor treatment of osteoarthritis. Sci. Transl. Med. 10(469), eaat8800 (2018). https:// doi.org/10.1126/scitranslmed.aat8800

41. A.G. Bajpayee, C.R. Wong, M.G. Bawendi, E.H. Frank, A.J. Grodzinsky, Avidin as a model for charge driven transport into cartilage and drug delivery for treating early stage post-traumatic osteoarthritis. Biomaterials 35(1), 538-549 (2014). https://doi.org/10.1016/j.biomaterials.2013.09.091

42. J. Malda, J.C. de Grauw, K.E. Benders, M.J. Kik, C.H. van de Lest et al., Of mice, men and elephants: the relation between articular cartilage thickness and body mass. PLoS ONE 8(2), e57683 (2013). https://doi.org/10.1371/journal. pone. 0057683

43. N. Kamisan, S.V. Naveen, R.E. Ahmad, T. Kamarul, Chondrocyte density, proteoglycan content and gene expressions from native cartilage are species specific and not dependent on cartilage thickness: a comparative analysis between rat, rabbit and goat. BMC Vet. Res. 9, 62 (2013). https://doi.org/ 10.1186/1746-6148-9-62

44. M. Morgen, D. Tung, B. Boras, W. Miller, A.M. Malfait et al., Nanoparticles for improved local retention after intra-articular injection into the knee joint. Pharm. Res. 30(1), 257-268 (2013). https://doi.org/10.1007/s11095-012-0870-x 
45. S. Brown, J. Pistiner, I.M. Adjei, B. Sharma, Nanoparticle properties for delivery to cartilage: the implications of disease state, synovial fluid, and off-target uptake. Mol. Pharm. 16(2), 469-479 (2019). https://doi.org/10.1021/acs.molph armaceut.7b00484

46. J. Schlessinger, A.N. Plotnikov, O.A. Ibrahimi, A.V. Eliseenkova, B.K. Yeh et al., Crystal structure of a ternary fgf-fgfrheparin complex reveals a dual role for heparin in fgfr binding and dimerization. Mol. Cell 6(3), 743-750 (2000). https:// doi.org/10.1016/s1097-2765(00)00073-3

47. L. Haglund, V. Tillgren, P. Onnerfjord, D. Heinegard, The c-terminal peptide of chondroadherin modulates cellular activity by selectively binding to heparan sulfate chains. J. Biol. Chem. 288(2), 995-1008 (2013). https://doi.org/10. 1074/jbc.M112.430512

48. T. Tokunou, R. Miller, P. Patwari, M.E. Davis, V.F. Segers et al., Engineering insulin-like growth factor-1 for local delivery. FASEB J. 22(6), 1886-1893 (2008). https://doi. org/10.1096/fj.07-100925

49. R.E. Miller, A.J. Grodzinsky, K. Cummings, A.H.K. Plaas, A.A. Cole et al., Intraarticular injection of heparin-binding insulin-like growth factor 1 sustains delivery of insulin-like growth factor 1 to cartilage through binding to chondroitin sulfate. Arthritis Rheum. 62(12), 3686-3694 (2010). https://doi.org/10.1002/art.27709

50. F.S. Loffredo, J.R. Pancoast, L. Cai, T. Vannelli, J.Z. Dong et al., Targeted delivery to cartilage is critical for in vivo efficacy of insulin-like growth factor 1 in a rat model of osteoarthritis. Arthritis Rheum. 66(5), 1247-1255 (2014). https://doi.org/10.1002/art.38357

51. H. Cho, J.M. Stuart, R. Magid, D.C. Danila, T. Hunsaker et al., Theranostic immunoliposomes for osteoarthritis. Nanomed.-Nanotechnol. Biol. Med. 10(3), 619-627 (2014). https://doi.org/10.1016/j.nano.2013.09.004

52. J. Hwang, Y. Huang, T.J. Burwell, N.C. Peterson, J. Connor, S.J. Weiss, S.M. Yu, Y. Li, In situ imaging of tissue remodeling with collagen hybridizing peptides. ACS Nano 11(10), 9825-9835 (2017). https://doi.org/10.1021/acsna no. $7 \mathrm{~b} 03150$

53. H. Chen, Z. Qin, J. Zhao, Y. He, E. Ren et al., Cartilagetargeting and dual mmp-13/ph responsive theranostic nanoprobes for osteoarthritis imaging and precision therapy. Biomaterials 225, 119520 (2019). https://doi.org/10.1016/j. biomaterials.2019.119520

54. S.B. Brown, L. Wang, R.R. Jungels, B. Sharma, Effects of cartilage-targeting moieties on nanoparticle biodistribution in healthy and osteoarthritic joints. Acta Biomater. 101, 469-483 (2020). https://doi.org/10.1016/j.actbio.2019.10.003

55. T.M. Quinn, A.A. Maung, A.J. Grodzinsky, E.B. Hunziker, J.D. Sandy, Physical and biological regulation of proteoglycan turnover around chondrocytes in cartilage explants. Implications for tissue degradation and repair. Ann. N. Y. Acad. Sci. 878, 420-441 (1999). https://doi.org/10.1111/j. 1749-6632.1999.tb07700.x

56. C.A. Poole, A. Matsuoka, J.R. Schofield, Chondrons from articular cartilage. Iii. Morphologic changes in the cellular microenvironment of chondrons isolated from osteoarthritic cartilage. Arthritis Rheum. 34(1), 22-35 (1991). https://doi. org/10.1002/art.1780340105

57. H. Laroui, L. Grossin, M. Léonard, J.F. Stoltz, P. Gillet et al., Hyaluronate-covered nanoparticles for the therapeutic targeting of cartilage. Biomacromol 8(12), 3879-3885 (2007). https://doi.org/10.1021/bm700836y

58. C.B. Knudson, W. Knudson, Hyaluronan and cd44: modulators of chondrocyte metabolism. Clin. Orthop. Relat. Res. 427(Suppl), S152-162 (2004)

59. Z. Chen, J. Chen, L. Wu, W. Li, J. Chen et al., Hyaluronic acid-coated bovine serum albumin nanoparticles loaded with brucine as selective nanovectors for intra-articular injection. Int. J. Nanomed. 8, 3843-3853 (2013). https://doi.org/10. 2147/IJN.S50721

60. N.D. Donahue, H. Acar, S. Wilhelm, Concepts of nanoparticle cellular uptake, intracellular trafficking, and kinetics in nanomedicine. Adv. Drug Deliv. Rev. 143, 68-96 (2019). https://doi.org/10.1016/j.addr.2019.04.008

61. W. He, X. Xing, X. Wang, D. Wu, W. Wu et al., Nanocarriermediated cytosolic delivery of biopharmaceuticals. Adv. Funct. Mater. 30, 1910566 (2020). https://doi.org/10.1002/ adfm.201910566

62. A.K. Gaharwar, I. Singh, A. Khademhosseini, Engineered biomaterials for in situ tissue regeneration. Nat. Rev. Mater. 5, 686-705 (2020). https://doi.org/10.1038/s41578-020-0209-x

63. B.C. te Boekhorst, L.B. Jensen, S. Colombo, A.K. Varkouhi, R.M. Schiffelers et al., Mri-assessed therapeutic effects of locally administered plga nanoparticles loaded with antiinflammatory sirna in a murine arthritis model. J. Control. Release 161(3), 772-780 (2012). https://doi.org/10.1016/j. jconrel.2012.05.004

64. J.S. Park, H.N. Yang, S.Y. Jeon, D.G. Woo, M.S. Kim et al., The use of anti-COX2 sirna coated onto plga nanoparticles loading dexamethasone in the treatment of rheumatoid arthritis. Biomaterials 33(33), 8600-8612 (2012). https://doi.org/ 10.1016/j.biomaterials.2012.08.008

65. Y. Wang, P. Li, L. Kong, Chitosan-modified plga nanoparticles with versatile surface for improved drug delivery. AAPS PharmSciTech 14(2), 585-592 (2013). https://doi.org/10. 1208/s12249-013-9943-3

66. N. Nafee, S. Taetz, M. Schneider, U.F. Schaefer, C.M. Lehr, Chitosan-coated plga nanoparticles for DNA/rna delivery: effect of the formulation parameters on complexation and transfection of antisense oligonucleotides. Nanomedicine 3(3), 173-183 (2007). https://doi.org/10.1016/j.nano.2007. 03.006

67. F. Esmaeili, M.H. Ghahremani, S.N. Ostad, F. Atyabi, M. Seyedabadi et al., Folate-receptor-targeted delivery of docetaxel nanoparticles prepared by plga-peg-folate conjugate. J. Drug Target. 16(5), 415-423 (2008). https://doi.org/10.1080/ 10611860802088630

68. K. Zhang, X. Tang, J. Zhang, W. Lu, X. Lin et al., Peg-plga copolymers: their structure and structure-influenced drug delivery applications. J. Control. Release 183, 77-86 (2014). https://doi.org/10.1016/j.jconrel.2014.03.026 
69. J.S. Suk, Q. Xu, N. Kim, J. Hanes, L.M. Ensign, Pegylation as a strategy for improving nanoparticle-based drug and gene delivery. Adv. Drug Deliv. Rev. 99(Pt A), 28-51 (2016). https://doi.org/10.1016/j.addr.2015.09.012

70. M. Fathi-Achachelouei, D. Keskin, E. Bat, N.E. Vrana, A. Tezcaner, Dual growth factor delivery using plga nanoparticles in silk fibroin/pegdma hydrogels for articular cartilage tissue engineering. J. Biomed. Mater. Res. Part B 108(5), 2041-2062 (2020). https://doi.org/10.1002/jbm.b.34544

71. J.H. Kim, J.S. Park, H.N. Yang, D.G. Woo, S.Y. Jeon et al., The use of biodegradable PLGA nanoparticles to mediate $S O X 9$ gene delivery in human mesenchymal stem cells (hMSCs) and induce chondrogenesis. Biomaterials 32(1), 268-278 (2011). https://doi.org/10.1016/j.biomaterials.2010. 08.086

72. Y.N. Tan, P.P. Lee, W.N. Chen, Microbial extraction of chitin from seafood waste using sugars derived from fruit wastestream. AMB Express 10(1), 17-17 (2020). https://doi.org/ 10.1186/s13568-020-0954-7

73. R. Jayakumar, K.P. Chennazhi, S. Srinivasan, S.V. Nair, T. Furuike et al., Chitin scaffolds in tissue engineering. Int. J. Mol. Sci. 12(3), 1876-1887 (2011). https://doi.org/10.3390/ ijms 12031876

74. R.C.F. Cheung, T.B. Ng, J.H. Wong, W.Y. Chan, Chitosan: an update on potential biomedical and pharmaceutical applications. Mar. Drugs 13(8), 5156-5186 (2015). https://doi.org/ 10.3390/md13085156

75. H. Tan, C.R. Chu, K.A. Payne, K.G. Marra, Injectable in situ forming biodegradable chitosan-hyaluronic acid based hydrogels for cartilage tissue engineering. Biomaterials 30(13), 2499-2506 (2009). https://doi.org/10.1016/j.biomaterials. 2008.12.080

76. C. Manferdini, E. Gabusi, L. Sartore, K. Dey, S. Agnelli et al., Chitosan-based scaffold counteracts hypertrophic and fibrotic markers in chondrogenic differentiated mesenchymal stromal cells. J. Tissue Eng. Regen. Med. 13(10), 1896-1911 (2019). https://doi.org/10.1002/term.2941

77. J.K. Suh, H.W. Matthew, Application of chitosan-based polysaccharide biomaterials in cartilage tissue engineering: a review. Biomaterials 21(24), 2589-2598 (2000). https://doi. org/10.1016/s0142-9612(00)00126-5

78. M.M. Islam, M. Shahruzzaman, S. Biswas, M.N. Sakib, T.U. Rashid, Chitosan based bioactive materials in tissue engineering applications-a review. Bioact. Mater. 5(1), 164-183 (2020). https://doi.org/10.1016/j.bioactmat.2020.01.012

79. K.Y. Lee, W.S. Ha, W.H. Park, Blood compatibility and biodegradability of partially n-acylated chitosan derivatives. Biomaterials 16(16), 1211-1216 (1995). https://doi.org/10. 1016/0142-9612(95)98126-y

80. H. Katas, M.A.G. Raja, K.L. Lam, Development of chitosan nanoparticles as a stable drug delivery system for protein/ siRNA. Int. J. Biomater. 2013, 146320-146320 (2013). https://doi.org/10.1155/2013/146320
81. S. Mao, W. Sun, T. Kissel, Chitosan-based formulations for delivery of DNA and siRNA. Adv. Drug Deliv. Rev. 62(1), 12-27 (2010). https://doi.org/10.1016/j.addr.2009.08.004

82. Y. Zhou, S.Q. Liu, H. Peng, L. Yu, B. He et al., In vivo antiapoptosis activity of novel berberine-loaded chitosan nanoparticles effectively ameliorates osteoarthritis. Int. Immunopharmacol. 28(1), 34-43 (2015). https://doi.org/10.1016/j. intimp.2015.05.014

83. Q. Shi, E.P. Rondon-Cavanzo, I.P.D. Picola, M.J. Tiera, X. Zhang et al., In vivo therapeutic efficacy of tnf $\alpha$ silencing by folate-peg-chitosan-deae/siRNA nanoparticles in arthritic mice. Int. J. Nanomed. 13, 387-402 (2018). https://doi.org/ 10.2147/ijn.S146942

84. H. Lu, L. Lv, Y. Dai, G. Wu, H. Zhao et al., Porous chitosan scaffolds with embedded hyaluronic acid/chitosan/plasmidDNA nanoparticles encoding TGF- $\beta 1$ induce DNA controlled release, transfected chondrocytes, and promoted cell proliferation. PLoS ONE 8(7), e69950 (2013). https://doi.org/10. 1371/journal.pone.0069950

85. S.J. Lee, A. Lee, S.R. Hwang, J.S. Park, J. Jang et al., TNF- $\alpha$ gene silencing using polymerized siRNA/thiolated glycol chitosan nanoparticles for rheumatoid arthritis. Mol. Ther. 22(2), 397-408 (2014). https://doi.org/10.1038/mt.2013.245

86. E. Lallana, J.M. Rios de la Rosa, A. Tirella, M. Pelliccia, A. Gennari et al., Chitosan/hyaluronic acid nanoparticles: rational design revisited for rna delivery. Mol. Pharm. 14(7), 2422-2436 (2017). https://doi.org/10.1021/acs.molpharmac eut.7b00320

87. P.H. Zhou, B. Qiu, R.H. Deng, H.J. Li, X.F. Xu et al., Chondroprotective effects of hyaluronic acid-chitosan nanoparticles containing plasmid DNA encoding cytokine response modifier a in a rat knee osteoarthritis model. Cell. Physiol. Biochem. 47(3), 1207-1216 (2018). https://doi.org/10.1159/ 000490217

88. Y. Cao, Y.F. Tan, Y.S. Wong, M.W.J. Liew, S. Venkatraman, Recent advances in chitosan-based carriers for gene delivery. Mar. Drugs 17(6), 381 (2019). https://doi.org/10.3390/md170 60381

89. X. Ji, Y. Yan, T. Sun, Q. Zhang, Y. Wang et al., Glucosamine sulphate-loaded distearoyl phosphocholine liposomes for osteoarthritis treatment: combination of sustained drug release and improved lubrication. Biomater. Sci. 7(7), 27162728 (2019). https://doi.org/10.1039/c9bm00201d

90. J.M. van den Hoven, S.R. Van Tomme, J.M. Metselaar, B. Nuijen, J.H. Beijnen et al., Liposomal drug formulations in the treatment of rheumatoid arthritis. Mol. Pharm. 8(4), 1002-1015 (2011). https://doi.org/10.1021/mp2000742

91. S. Sivan, A. Schroeder, G. Verberne, Y. Merkher, D. Diminsky et al., Liposomes act as effective biolubricants for friction reduction in human synovial joints. Langmuir 26(2), 1107-1116 (2010). https://doi.org/10.1021/la9024712

92. J. Dong, D. Jiang, Z. Wang, G. Wu, L. Miao et al., Intraarticular delivery of liposomal celecoxib-hyaluronate combination for the treatment of osteoarthritis in rabbit model. 
Int. J. Pharm. 441(1-2), 285-290 (2013). https://doi.org/10. 1016/j.ijpharm.2012.11.031

93. I. Elron-Gross, Y. Glucksam, R. Margalit, Liposomal dexamethasone-diclofenac combinations for local osteoarthritis treatment. Int. J. Pharm. 376(1-2), 84-91 (2009). https://doi. org/10.1016/j.ijpharm.2009.04.025

94. F.H. Chen, K.T. Rousche, R.S. Tuan, Technology insight: adult stem cells in cartilage regeneration and tissue engineering. Nat. Clin. Pract. Rheumatol. 2(7), 373-382 (2006). https://doi.org/10.1038/ncprheum0216

95. H. Watanabe, Y. Yamada, K. Kimata, Roles of aggrecan, a large chondroitin sulfate proteoglycan, in cartilage structure and function. J. Biochem. 124(4), 687-693 (1998). https:// doi.org/10.1093/oxfordjournals.jbchem.a022166

96. T.M. Tamer, Hyaluronan and synovial joint: function, distribution and healing. Interdiscip. Toxicol. 6(3), 111-125 (2013). https://doi.org/10.2478/intox-2013-0019

97. R.C. Gupta, R. Lall, A. Srivastava, A. Sinha, Hyaluronic acid: molecular mechanisms and therapeutic trajectory. Front. Vet. Sci. 6, 192 (2019). https://doi.org/10.3389/fvets.2019.00192

98. E. George, Intra-articular hyaluronan treatment for osteoarthritis. Ann. Rheum. Dis. 57(11), 637-640 (1998). https:// doi.org/10.1136/ard.57.11.637

99. P.A. Band, J. Heeter, H.G. Wisniewski, V. Liublinska, C.W. Pattanayak et al., Hyaluronan molecular weight distribution is associated with the risk of knee osteoarthritis progression. Osteoarthritis Cartil. 23(1), 70-76 (2015). https://doi. org/10.1016/j.joca.2014.09.017

100. E.A. Balazs, J.L. Denlinger, Viscosupplementation: a new concept in the treatment of osteoarthritis. J. Rheumatol. Suppl. 39, 3-9 (1993)

101. H.D. Lu, H.Q. Zhao, K. Wang, L.L. Lv, Novel hyaluronic acid-chitosan nanoparticles as non-viral gene delivery vectors targeting osteoarthritis. Int. J. Pharm. 420(2), 358-365 (2011). https://doi.org/10.1016/j.ijpharm.2011.08.046

102. M. Zhou, J. Hou, Z. Zhong, N. Hao, Y. Lin et al., Targeted delivery of hyaluronic acid-coated solid lipid nanoparticles for rheumatoid arthritis therapy. Drug Deliv. 25(1), 716-722 (2018). https://doi.org/10.1080/10717544.2018. 1447050

103. H. Lu, Y. Dai, L. Lv, H. Zhao, Chitosan-graft-polyethylenimine/DNA nanoparticles as novel non-viral gene delivery vectors targeting osteoarthritis. PLoS ONE 9(1), e84703 (2014). https://doi.org/10.1371/journal.pone.0084703

104. S. Nedvetzki, M. Walmsley, E. Alpert, R.O. Williams, M. Feldmann et al., Cd44 involvement in experimental collageninduced arthritis (CIA). J. Autoimmun. 13(1), 39-47 (1999). https://doi.org/10.1006/jaut.1999.0294

105. G.M. Campo, A. Avenoso, S. Campo, A. D’Ascola, G. Nastasi et al., Small hyaluronan oligosaccharides induce inflammation by engaging both toll-like-4 and CD44 receptors in human chondrocytes. Biochem. Pharmacol. 80(4), 480-490 (2010). https://doi.org/10.1016/j.bcp.2010.04.024

106. F.J. Zhang, S.G. Gao, L. Cheng, J. Tian, W.S. Xu et al., The effect of hyaluronic acid on osteopontin and CD44 mrna of fibroblast-like synoviocytes in patients with osteoarthritis of the knee. Rheumatol. Int. 33(1), 79-83 (2013). https://doi. org/10.1007/s00296-011-2339-3

107. P. Maudens, S. Meyer, C.A. Seemayer, O. Jordan, E. Allémann, Self-assembled thermoresponsive nanostructures of hyaluronic acid conjugates for osteoarthritis therapy. Nanoscale 10(4), 1845-1854 (2018). https://doi.org/10.1039/ c7nr07614b

108. J.E. Rowley, F. Amargant, L.T. Zhou, A. Galligos, L.E. Simon et al., Low molecular weight hyaluronan induces an inflammatory response in ovarian stromal cells and impairs gamete development in vitro. Int. J. Mol. Sci. 21(3), 1036 (2020). https://doi.org/10.3390/ijms21031036

109. J. Monfort, J.P. Pelletier, N. Garcia-Giralt, J. Martel-Pelletier, Biochemical basis of the effect of chondroitin sulphate on osteoarthritis articular tissues. Ann. Rheum. Dis. 67(6), 735-740 (2008). https://doi.org/10.1136/ard.2006.068882

110. R.M. Lauder, Chondroitin sulphate: a complex molecule with potential impacts on a wide range of biological systems. Complement. Ther. Med. 17(1), 56-62 (2009). https://doi. org/10.1016/j.ctim.2008.08.004

111. P.J. Roughley, The structure and function of cartilage proteoglycans. Eur. Cells Mater. 12, 92-101 (2006). https:// doi.org/10.22203/ecm.v012a11

112. U.P. Palukuru, C.M. McGoverin, N. Pleshko, Assessment of hyaline cartilage matrix composition using near infrared spectroscopy. Matrix Biol. 38, 3-11 (2014). https://doi.org/ 10.1016/j.matbio.2014.07.007

113. L. Bian, M. Kaplun, D.Y. Williams, D. Xu, G.A. Ateshian et al., Influence of chondroitin sulfate on the biochemical, mechanical and frictional properties of cartilage explants in long-term culture. J. Biomech. 42(3), 286-290 (2009). https://doi.org/10.1016/j.jbiomech.2008.10.032

114. A.I. Maroudas, Balance between swelling pressure and collagen tension in normal and degenerate cartilage. Nature 260(5554), 808-809 (1976). https://doi.org/10.1038/26080 $8 \mathrm{a} 0$

115. M. David-Raoudi, B. Deschrevel, S. Leclercq, P. Galera, K. Boumediene et al., Chondroitin sulfate increases hyaluronan production by human synoviocytes through differential regulation of hyaluronan synthases: role of p38 and akt. Arthritis Rheum. 60(3), 760-770 (2009). https://doi.org/ 10.1002/art.24302

116. Q.G. Wang, A.J. El. Haj, N.J. Kuiper, Glycosaminoglycans in the pericellular matrix of chondrons and chondrocytes. J. Anat. 213(3), 266-273 (2008). https://doi.org/10.1111/j. 1469-7580.2008.00942.x

117. M. Bishnoi, A. Jain, P. Hurkat, S.K. Jain, Chondroitin sulphate: a focus on osteoarthritis. Glycoconjugate J. 33(5), 693-705 (2016). https://doi.org/10.1007/ s10719-016-9665-3

118. V. Calamia, L. Lourido, P. Fernandez-Puente, J. Mateos, B. Rocha et al., Secretome analysis of chondroitin sulfate-treated chondrocytes reveals anti-angiogenic, anti-inflammatory and anti-catabolic properties. Arthritis Res. Ther. 14(5), R202 (2012). https://doi.org/10.1186/ar4040 
119. D. Ishimaru, N. Sugiura, H. Akiyama, H. Watanabe, K. Matsumoto, Alterations in the chondroitin sulfate chain in human osteoarthritic cartilage of the knee. Osteoarthritis Cartil. 22(2), 250-258 (2014). https://doi.org/10.1016/j.joca.2013. 11.010

120. Y.K. Jung, H.R. Park, H.J. Cho, J.A. Jang, E.J. Lee et al., Degrading products of chondroitin sulfate can induce hypertrophy-like changes and MMP-13/ADAMTS5 production in chondrocytes. Sci. Rep. 9(1), 15846 (2019). https://doi.org/ 10.1038/s41598-019-52358-4

121. K.M. Jordan, N.K. Arden, M. Doherty, B. Bannwarth, J.W. Bijlsma et al., Eular recommendations 2003: an evidence based approach to the management of knee osteoarthritis: report of a task force of the standing committee for international clinical studies including therapeutic trials (escisit). Ann. Rheum. Dis. 62(12), 1145-1155 (2003). https://doi.org/ 10.1136/ard.2003.011742

122. A. Jain, S.K. Mishra, P.R. Vuddanda, S.K. Singh, R. Singh et al., Targeting of diacerein loaded lipid nanoparticles to intra-articular cartilage using chondroitin sulfate as homing carrier for treatment of osteoarthritis in rats. Nanomed.Nanotechnol. Biol. Med. 10(5), 1031-1040 (2014). https:// doi.org/10.1016/j.nano.2014.01.008

123. X.F. Yin, L.L. Wang, X.C. Chu, A novel chondroitin sulfate decorated nano platinum for the treatment of osteoarthritis. Mater. Sci. Eng. C-Mater. Biol. Appl. 78, 452-456 (2017). https://doi.org/10.1016/j.msec.2017.04.028

124. F. Bakilan, O. Armagan, M. Ozgen, F. Tascioglu, O. Bolluk et al., Effects of native type ii collagen treatment on knee osteoarthritis: a randomized controlled trial. Eurasian J. Med. 48(2), 95-101 (2016). https://doi.org/10.5152/euras ianjmed.2015.15030

125. C. Lian, X. Wang, X. Qiu, Z. Wu, B. Gao et al., Collagen type ii suppresses articular chondrocyte hypertrophy and osteoarthritis progression by promoting integrin $\beta 1$-smad 1 interaction. Bone Res. (2019). https://doi.org/10.1038/ s41413-019-0046-y

126. G.S. Hussey, J.L. Dziki, S.F. Badylak, Extracellular matrix-based materials for regenerative medicine. Nat. Rev. Mater. 3(7), 159-173 (2018). https://doi.org/10.1038/ s41578-018-0023-x

127. K.E. Benders, P.R. van Weeren, S.F. Badylak, D.B. Saris, W.J. Dhert et al., Extracellular matrix scaffolds for cartilage and bone regeneration. Trends Biotechnol. 31(3), 169-176 (2013). https://doi.org/10.1016/j.tibtech.2012.12.004

128. X. Li, W. Guo, K. Zha, X. Jing, M. Wang et al., Enrichment of CD146(+) adipose-derived stem cells in combination with articular cartilage extracellular matrix scaffold promotes cartilage regeneration. Theranostics 9(17), 51055121 (2019). https://doi.org/10.7150/thno.33904

129. Y. Zhang, S. Liu, W. Guo, M. Wang, C. Hao et al., Human umbilical cord wharton's jelly mesenchymal stem cells combined with an acellular cartilage extracellular matrix scaffold improve cartilage repair compared with microfracture in a caprine model. Osteoarthritis Cartil. 26(7), 954-965 (2018). https://doi.org/10.1016/j.joca.2018.01.019
130. S. Zahiri, E. Masaeli, E. Poorazizi, M.H. Nasr-Esfahani, Chondrogenic response in presence of cartilage extracellular matrix nanoparticles. J. Biomed. Mater. Res. Part A 106(9), 2463-2471 (2018). https://doi.org/10.1002/jbm.a. 36440

131. D. Shi, X. Xu, Y. Ye, K. Song, Y. Cheng et al., Photo-crosslinked scaffold with kartogenin-encapsulated nanoparticles for cartilage regeneration. ACS Nano 10(1), 1292-1299 (2016). https://doi.org/10.1021/acsnano.5b06663

132. W. Zhang, C. Ling, A. Zhang, H. Liu, Y. Jiang et al., An all-silk-derived functional nanosphere matrix for sequential biomolecule delivery and in situ osteochondral regeneration. Bioact. Mater. 5(4), 832-843 (2020). https://doi.org/10. 1016/j.bioactmat.2020.05.003

133. J. Shi, X. Zhang, J. Zhu, Y. Pi, X. Hu et al., Nanoparticle delivery of the bone morphogenetic protein 4 gene to adipose-derived stem cells promotes articular cartilage repair in vitro and in vivo. Arthrosc.: J. Arthrosc. Relat. Surg.: Off. Publ. Arthrosc. Assoc. N. Am. Int. Arthrosc. Assoc. 29(12), 2001-2011.e2002 (2013). https://doi.org/10.1016/j.arthro. 2013.09.076

134. J. Li, Z. Li, D. Chu, L. Jin, X. Zhang, Fabrication and biocompatibility of core-shell structured magnetic fibrous scaffold. J. Biomed. Nanotechnol. 15(3), 500-506 (2019). https:// doi.org/10.1166/jbn.2019.2701

135. Z. Li, D. Chu, G. Chen, L. Shi, L. Jin et al., Biocompatible and biodegradable $3 \mathrm{~d}$ double-network fibrous scaffold for excellent cell growth. J. Biomed. Nanotechnol. 15(11), 2209-2215 (2019). https://doi.org/10.1166/jbn.2019.2846

136. R. Huang, X. Chen, Y. Dong, X. Zhang, Y. Wei et al., Mxene composite nanofibers for cell culture and tissue engineering. ACS Appl. Bio. Mater. 3(4), 2125-2131 (2020). https://doi. org/10.1021/acsabm.0c00007

137. C. Boyer, L. Figueiredo, R. Pace, J. Lesoeur, T. Rouillon et al., Laponite nanoparticle-associated silated hydroxypropylmethyl cellulose as an injectable reinforced interpenetrating network hydrogel for cartilage tissue engineering. Acta Biomater. (2018). https://doi.org/10.1016/j.actbio.2017.11. 027

138. H. Meng, Q. Quan, X. Yuan, Y. Zheng, J. Peng et al., Diffusion of neutral solutes within human osteoarthritic cartilage: effect of loading patterns. J. Orthop. Transl. 22, 58-66 (2020). https://doi.org/10.1016/j.jot.2019.10.013

139. Z. Sun, E. Feeney, Y. Guan, S.G. Cook, D. Gourdon et al., Boundary mode lubrication of articular cartilage with a biomimetic diblock copolymer. Proc. Natl. Acad. Sci. U. S. A. 116(25), 12437-12441 (2019). https://doi.org/10.1073/pnas. 1900716116

140. Y. Yan, T. Sun, H. Zhang, X. Ji, Y. Sun et al., Euryale ferox seed-inspired superlubricated nanoparticles for treatment of osteoarthritis. Adv. Funct. Mater. 29(4), 1807559 (2019). https://doi.org/10.1002/adfm.201807559

141. P.M. van der Kraan, W.B. van den Berg, Chondrocyte hypertrophy and osteoarthritis: role in initiation and progression of cartilage degeneration? Osteoarthritis Cartil. 20(3), 223-232 (2012). https://doi.org/10.1016/j.joca.2011.12.003 
142. S. Chen, P. Fu, R. Cong, H. Wu, M. Pei, Strategies to minimize hypertrophy in cartilage engineering and regeneration. Genes Dis. 2(1), 76-95 (2015). https://doi.org/10.1016/j.gendis.2014.12.003

143. H. Sasaki, K. Takayama, T. Matsushita, K. Ishida, S. Kubo et al., Autophagy modulates osteoarthritis-related gene expression in human chondrocytes. Arthritis Rheum. 64(6), 1920-1928 (2012). https://doi.org/10.1002/art.34323

144. R.F. Loeser, J.A. Collins, B.O. Diekman, Ageing and the pathogenesis of osteoarthritis. Nat. Rev. Rheumatol. 12(7), 412-420 (2016). https://doi.org/10.1038/nrrheum.2016.65

145. G. Zhong, X. Yang, X. Jiang, A. Kumar, H. Long et al., Dopamine-melanin nanoparticles scavenge reactive oxygen and nitrogen species and activate autophagy for osteoarthritis therapy. Nanoscale 11(24), 11605-11616 (2019). https://doi. org/10.1039/c9nr03060c

146. H. Yan, X. Duan, H. Pan, N. Holguin, M.F. Rai et al., Suppression of NF- $\kappa$ B activity via nanoparticle-based sirna delivery alters early cartilage responses to injury. Proc. Natl. Acad. Sci. U. S. A. 113(41), E6199-e6208 (2016). https://doi.org/ 10.1073/pnas.1608245113

147. P.G. Conaghan, A.D. Cook, J.A. Hamilton, P.P. Tak, Therapeutic options for targeting inflammatory osteoarthritis pain. Nat. Rev. Rheumatol. 15(6), 355-363 (2019). https://doi.org/ 10.1038/s41584-019-0221-y

148. H.-F. Zhou, H. Yan, A. Senpan, S.A. Wickline, D. Pan et al., Suppression of inflammation in a mouse model of rheumatoid arthritis using targeted lipase-labile fumagillin prodrug nanoparticles. Biomaterials 33(33), 8632-8640 (2012). https://doi. org/10.1016/j.biomaterials.2012.08.005

149. M. Kapoor, J. Martel-Pelletier, D. Lajeunesse, J.P. Pelletier, H. Fahmi, Role of proinflammatory cytokines in the pathophysiology of osteoarthritis. Nat. Rev. Rheumatol. 7(1), 33-42 (2011). https://doi.org/10.1038/nrrheum.2010.196

150. R.E. Whitmire, D.S. Wilson, A. Singh, M.E. Levenston, N. Murthy et al., Self-assembling nanoparticles for intra-articular delivery of anti-inflammatory proteins. Biomaterials 33(30), 7665-7675 (2012). https://doi.org/10.1016/j.bioma terials.2012.06.101

151. H. Lee, M.Y. Lee, S.H. Bhang, B.S. Kim, Y.S. Kim et al., Hyaluronate-gold nanoparticle/tocilizumab complex for the treatment of rheumatoid arthritis. ACS Nano 8(5), 4790-4798 (2014). https://doi.org/10.1021/nn500685h

152. M.R. Bhalekar, P.G. Upadhaya, A.R. Madgulkar, Fabrication and efficacy evaluation of chloroquine nanoparticles in cfainduced arthritic rats using TNF- $\alpha$ elisa. Eur. J. Pharm. Sci. 84, 1-8 (2016). https://doi.org/10.1016/j.ejps.2016.01.009

153. J.B. Lin, S. Poh, A. Panitch, Controlled release of anti-inflammatory peptides from reducible thermosensitive nanoparticles suppresses cartilage inflammation. Nanomed. Nanotechnol. Biol. Med. 12(7), 2095-2100 (2016). https://doi.org/10. 1016/j.nano.2016.05.010

154. R.L. Bartlett 2nd., S. Sharma, A. Panitch, Cell-penetrating peptides released from thermosensitive nanoparticles suppress pro-inflammatory cytokine response by specifically targeting inflamed cartilage explants. Nanomedicine $\mathbf{9}(3)$, 419-427 (2013). https://doi.org/10.1016/j.nano.2012.09.003

155. J. McMasters, S. Poh, J.B. Lin, A. Panitch, Delivery of antiinflammatory peptides from hollow PEGylated poly(NIPAM) nanoparticles reduces inflammation in an ex vivo osteoarthritis model. J. Control. Release 258, 161-170 (2017). https:// doi.org/10.1016/j.jconrel.2017.05.008

156. L. Yuan, R.-X. Liao, Y.-Y. Lin, Y. Jiang, O. Wang et al., Safety and efficacy of cyclooxygenase-2 inhibition for treatment of primary hypertrophic osteoarthropathy: a single-arm intervention trial. J. Orthop. Transl. 18, 109-118 (2018). https://doi.org/10.1016/j.jot.2018.10.001

157. M.J. Kim, J.S. Park, S.J. Lee, J. Jang, J.S. Park et al., Notch1 targeting siRNA delivery nanoparticles for rheumatoid arthritis therapy. J. Control. Release 216, 140-148 (2015). https:// doi.org/10.1016/j.jconrel.2015.08.025

158. P. Lepetsos, A.G. Papavassiliou, Ros/oxidative stress signaling in osteoarthritis. Biochim. Biophys. Acta 1862(4), 576591 (2016). https://doi.org/10.1016/j.bbadis.2016.01.003

159. Y.C. Cheuk, S.C. Fu, S.W. Mok, K.K. Ho, L.K. Hung et al., Intra-articular injection of an antioxidant formulation did not improve structural degeneration in a rat model of posttraumatic osteoarthritis. J. Orthop. Transl. 8, 25-31 (2017). https://doi.org/10.1016/j.jot.2016.08.001

160. W. Li, L. Cai, Y. Zhang, L. Cui, G. Shen, Intra-articular resveratrol injection prevents osteoarthritis progression in a mouse model by activating SIRT1 and thereby silencing HIF-2 $\alpha$. J. Orthop. Res. 33(7), 1061-1070 (2015). https:// doi.org/10.1002/jor.22859

161. S. Kumar, I.M. Adjei, S.B. Brown, O. Liseth, B. Sharma, Manganese dioxide nanoparticles protect cartilage from inflammation-induced oxidative stress. Biomaterials 224, 119467 (2019). https://doi.org/10.1016/j.biomaterials.2019. 119467

162. L. Zhang, J. Hu, K.A. Athanasiou, The role of tissue engineering in articular cartilage repair and regeneration. Crit. Rev. Biomed. Eng. 37(1-2), 1-57 (2009). https://doi.org/10. 1615/critrevbiomedeng.v37.i1-2.10

163. S. Zhang, B. Hu, W. Liu, P. Wang, X. Lv et al., Articular cartilage regeneration: the role of endogenous mesenchymal stem/progenitor cell recruitment and migration. Semin. Arthritis Rheum. 50(2), 198-208 (2020). https://doi.org/10. 1016/j.semarthrit.2019.11.001

164. N. Dunker, K. Krieglstein, Targeted mutations of transforming growth factor-beta genes reveal important roles in mouse development and adult homeostasis. Eur. J. Biochem. 267(24), 6982-6988 (2000). https://doi.org/10.1046/j.14321327.2000.01825.x

165. J. Shen, J. Li, B. Wang, H. Jin, M. Wang et al., Deletion of the transforming growth factor beta receptor type ii gene in articular chondrocytes leads to a progressive osteoarthritislike phenotype in mice. Arthritis Rheum. 65(12), 3107-3119 (2013). https://doi.org/10.1002/art.38122

166. T. Li, S. Chubinskaya, A. Esposito, X. Jin, L. Tagliafierro et al., Tgf-beta type 2 receptor-mediated modulation of the il-36 family can be therapeutically targeted in osteoarthritis. 
Sci. Transl. Med. (2019). https://doi.org/10.1126/scitranslm ed.aan 2585

167. L.M. Cross, J.K. Carrow, X. Ding, K.A. Singh, A.K. Gaharwar, Sustained and prolonged delivery of protein therapeutics from two-dimensional nanosilicates. ACS Appl. Mater. Interfaces 11(7), 6741-6750 (2019). https://doi.org/10.1021/ acsami.8b17733

168. X. Sun, J. Wang, Y. Wang, C. Huang, C. Yang et al., Scaffold with orientated microtubule structure containing polylysineheparin sodium nanoparticles for the controlled release of tgf- $\beta 1$ in cartilage tissue engineering. ACS Appl. Bio Mater. 1(6), 2030-2040 (2018). https://doi.org/10.1021/acsabm. $8 \mathrm{~b} 00523$

169. F.Y. Wei, J.K. Lee, L. Wei, F. Qu, J.Z. Zhang, Correlation of insulin-like growth factor 1 and osteoarthritic cartilage degradation: a spontaneous osteoarthritis in guinea-pig. Eur. Rev. Med. Pharmacol. Sci. 21(20), 4493-4500 (2017)

170. K.J. Ekenstedt, W.E. Sonntag, R.F. Loeser, B.R. Lindgren, C.S. Carlson, Effects of chronic growth hormone and insulinlike growth factor 1 deficiency on osteoarthritis severity in rat knee joints. Arthritis Rheum. 54(12), 3850-3858 (2006). https://doi.org/10.1002/art.22254

171. J. Pasold, K. Zander, B. Heskamp, C. Grüttner, F. Lüthen et al., Positive impact of IGF-1-coupled nanoparticles on the differentiation potential of human chondrocytes cultured on collagen scaffolds. Int. J. Nanomed. 10, 1131-1143 (2015). https://doi.org/10.2147/ijn.S72872

172. L. Longobardi, L. O’Rear, S. Aakula, B. Johnstone, K. Shimer et al., Effect of IGF-i in the chondrogenesis of bone marrow mesenchymal stem cells in the presence or absence of TGF-beta signaling. J. Bone Miner. Res. 21(4), 626-636 (2006). https://doi.org/10.1359/jbmr.051213

173. J. Frisch, A. Rey-Rico, J.K. Venkatesan, G. Schmitt, H. Madry et al., Chondrogenic differentiation processes in human bone marrow aspirates upon raav-mediated gene transfer and overexpression of the insulin-like growth factor i. Tissue Eng. Part A 21(17-18), 2460-2471 (2015). https:// doi.org/10.1089/ten.TEA.2014.0679

174. M. Zhang, Q. Zhou, Q.Q. Liang, C.G. Li, J.D. Holz et al., IGF-1 regulation of type ii collagen and mmp-13 expression in rat endplate chondrocytes via distinct signaling pathways. Osteoarthritis Cartil. 17(1), 100-106 (2009). https://doi.org/ 10.1016/j.joca.2008.05.007

175. J.A. Tyler, Insulin-like growth factor 1 can decrease degradation and promote synthesis of proteoglycan in cartilage exposed to cytokines. Biochem. J. 260(2), 543-548 (1989). https://doi.org/10.1042/bj2600543

176. J.L. Ellsworth, J. Berry, T. Bukowski, J. Claus, A. Feldhaus et al., Fibroblast growth factor-18 is a trophic factor for mature chondrocytes and their progenitors. Osteoarthritis Cartil. 10(4), 308-320 (2002). https://doi.org/10.1053/joca. 2002.0514

177. E.E. Moore, A.M. Bendele, D.L. Thompson, A. Littau, K.S. Waggie et al., Fibroblast growth factor-18 stimulates chondrogenesis and cartilage repair in a rat model of injury-induced osteoarthritis. Osteoarthritis Cartil. 13(7), 623-631 (2005). https://doi.org/10.1016/j.joca.2005.03.003

178. S.L. Chia, Y. Sawaji, A. Burleigh, C. McLean, J. Inglis et al., Fibroblast growth factor 2 is an intrinsic chondroprotective agent that suppresses adamts- 5 and delays cartilage degradation in murine osteoarthritis. Arthritis Rheum. 60(7), 20192027 (2009). https://doi.org/10.1002/art.24654

179. X. Wang, P.A. Manner, A. Horner, L. Shum, R.S. Tuan et al., Regulation of MMP-13 expression by RUNX2 and FGF2 in osteoarthritic cartilage. Osteoarthritis Cartil. 12(12), 963-973 (2004). https://doi.org/10.1016/j.joca.2004.08.008

180. H.J. Im, X. Li, P. Muddasani, G.H. Kim, F. Davis et al., Basic fibroblast growth factor accelerates matrix degradation via a neuro-endocrine pathway in human adult articular chondrocytes. J. Cell. Physiol. 215(2), 452-463 (2008). https://doi. org/10.1002/jcp. 21317

181. D. Yan, D. Chen, S.M. Cool, A.J. van Wijnen, K. Mikecz et al., Fibroblast growth factor receptor 1 is principally responsible for fibroblast growth factor 2-induced catabolic activities in human articular chondrocytes. Arthritis Res. Ther. 13(4), R130 (2011). https://doi.org/10.1186/ar3441

182. N.D. Miljkovic, G.M. Cooper, K.G. Marra, Chondrogenesis, bone morphogenetic protein- 4 and mesenchymal stem cells. Osteoarthritis Cartil. 16(10), 1121-1130 (2008). https://doi.org/10.1016/j.joca.2008.03.003

183. C. Wang, R.M. Silverman, J. Shen, R.J. O'Keefe, Distinct metabolic programs induced by TGF- $\beta 1$ and bmp 2 in human articular chondrocytes with osteoarthritis. J. Orthop. Trans1. 12, 66-73 (2018). https://doi.org/10.1016/j.jot. 2017.12.004

184. B.S. Yoon, K.M. Lyons, Multiple functions of BMPS in chondrogenesis. J. Cell. Biochem. 93(1), 93-103 (2004). https://doi.org/10.1002/jcb.20211

185. K. Johnson, S. Zhu, M.S. Tremblay, J.N. Payette, J. Wang et al., A stem cell-based approach to cartilage repair. Science 336(6082), 717-721 (2012). https://doi.org/10.1126/ science. 1215157

186. Y. Hong, Y. Han, J. Wu, X. Zhao, J. Cheng et al., Chitosan modified $\mathrm{Fe}_{3} \mathrm{O}_{4} / \mathrm{KGN}$ self-assembled nanoprobes for osteochondral MR diagnose and regeneration. Theranostics 10(12), 5565-5577 (2020). https://doi.org/10.7150/thno.43569

187. S.P. Henry, S. Liang, K.C. Akdemir, B. de Crombrugghe, The postnatal role of SOX9 in cartilage. J. Bone Miner. Res. 27(12), 2511-2525 (2012). https://doi.org/10.1002/jbmr.1696

188. W. Li, J. Lin, Z. Wang, S. Ren, X. Wu et al., Bevacizumab tested for treatment of knee osteoarthritis via inhibition of synovial vascular hyperplasia in rabbits. J. Orthop. Transl. 19, 38-46 (2019). https://doi.org/10.1016/j.jot.2019.04.002

189. C.L. Wu, N.S. Harasymowicz, M.A. Klimak, K.H. Collins, F. Guilak, The role of macrophages in osteoarthritis and cartilage repair. Osteoarthritis Cartil. (2020). https://doi. org/10.1016/j.joca.2019.12.007

190. S. Li, W. Cong, A. Hakamivala, Y. Huang, J. Borrelli et al., Hyaluronic acid-based optical probe for the diagnosis of human osteoarthritic cartilage. Nanotheranostics 2(4), 347-359 (2018). https://doi.org/10.7150/ntno.26119 
191. Y. Yang, L. Guo, Z. Wang, P. Liu, X. Liu et al., Targeted silver nanoparticles for rheumatoid arthritis therapy via macrophage apoptosis and re-polarization. Biomaterials 264, 120390 (2021). https://doi.org/10.1016/j.biomateria 1s. 2020.120390

192. L. Ma, T.W. Liu, M.A. Wallig, I.T. Dobrucki, L.W. Dobrucki et al., Efficient targeting of adipose tissue macrophages in obesity with polysaccharide nanocarriers. ACS Nano 10(7), 6952-6962 (2016). https://doi.org/10.1021/acsnano.6b02878

193. G. Hu, M. Guo, J. Xu, F. Wu, J. Fan et al., Nanoparticles targeting macrophages as potential clinical therapeutic agents against cancer and inflammation. Front. Immunol. 10, 19981998 (2019). https://doi.org/10.3389/fimmu.2019.01998

194. M. Ovais, M. Guo, C. Chen, Tailoring nanomaterials for targeting tumor-associated macrophages. Adv. Mater. 31(19), e1808303 (2019). https://doi.org/10.1002/adma.201808303

195. J. Yang, X. Zhang, C. Liu, Z. Wang, L. Deng, C. Feng, W. Tao, X. Xu, W. Cui, Biologically modified nanoparticles as theranostic bionanomaterials. Prog. Mater. Sci. 118, 100768 (2021). https://doi.org/10.1016/j.pmatsci.2020.100768

196. R. Li, Y. He, Y. Zhu, L. Jiang, S. Zhang et al., Route to rheumatoid arthritis by macrophage-derived microvesiclecoated nanoparticles. Nano Lett. 19(1), 124-134 (2019). https://doi.org/10.1021/acs.nanolett.8b03439

197. Q. Zhang, D. Dehaini, Y. Zhang, J. Zhou, X. Chen et al., Neutrophil membrane-coated nanoparticles inhibit synovial inflammation and alleviate joint damage in inflammatory arthritis. Nat. Nanotechnol. 13(12), 1182-1190 (2018). https://doi.org/10.1038/s41565-018-0254-4

198. D.M. Findlay, J.S. Kuliwaba, Bone-cartilage crosstalk: a conversation for understanding osteoarthritis. Bone Res. 4, 16028 (2016). https://doi.org/10.1038/boneres.2016.28

199. R.J. Lories, F.P. Luyten, The bone-cartilage unit in osteoarthritis. Nat. Rev. Rheumatol. 7(1), 43-49 (2011). https://doi. org/10.1038/nrrheum.2010.197

200. L. Chu, Z. He, X. Qu, X. Liu, W. Zhang et al., Different subchondral trabecular bone microstructure and biomechanical properties between developmental dysplasia of the hip and primary osteoarthritis. J. Orthop. Transl. 22, 50-57 (2020). https://doi.org/10.1016/j.jot.2019.09.001

201. G. Li, L. Chen, Q. Zheng, Y. Ma, C. Zhang et al., Subchondral bone deterioration in femoral heads in patients with osteoarthritis secondary to hip dysplasia: a case-control study. J. Orthop. Transl. 24, 190-197 (2019). https://doi.org/10. 1016/j.jot.2019.10.014

202. S. Zhu, J. Zhu, G. Zhen, Y. Hu, S. An et al., Subchondral bone osteoclasts induce sensory innervation and osteoarthritis pain. J. Clin. Invest. 129(3), 1076-1093 (2019). https:// doi.org/10.1172/JCI121561

203. R. Kc, X. Li, J.S. Kroin, Z. Liu, D. Chen et al., PKCס null mutations in a mouse model of osteoarthritis alter osteoarthritic pain independently of joint pathology by augmenting NGF/TRKA-induced axonal outgrowth. Ann. Rheum. Dis. 75(12), 2133-2141 (2016). https://doi.org/10.1136/annrh eumdis-2015-208444
204. M. Ikeuchi, Q. Wang, M. Izumi, T. Tani, Nociceptive sensory innervation of the posterior cruciate ligament in osteoarthritic knees. Arch. Orthop. Trauma Surg. 132(6), 891-895 (2012). https://doi.org/10.1007/s00402-012-1478-7

205. A.M. Malfait, T.J. Schnitzer, Towards a mechanism-based approach to pain management in osteoarthritis. Nat. Rev. Rheumatol. 9(11), 654-664 (2013). https://doi.org/10.1038/ nrrheum.2013.138

206. M.P. Kashyap, C. Roberts, M. Waseem, P. Tyagi, Drug targets in neurotrophin signaling in the central and peripheral nervous system. Mol. Neurobiol. 55(8), 6939-6955 (2018). https://doi.org/10.1007/s12035-018-0885-3

207. S. Takano, K. Uchida, G. Inoue, M. Miyagi, J. Aikawa et al., Nerve growth factor regulation and production by macrophages in osteoarthritic synovium. Clin. Exp. Immunol. 190(2), 235-243 (2017). https://doi.org/10.1111/cei.13007

208. P. Jayabalan, T.J. Schnitzer, Tanezumab in the treatment of chronic musculoskeletal conditions. Expert Opin. Biol. Ther. 17(2), 245-254 (2017). https://doi.org/10.1080/14712598. 2017.1271873

209. L.N. Nwosu, P.I. Mapp, V. Chapman, D.A. Walsh, Blocking the tropomyosin receptor kinase a (TRKA) receptor inhibits pain behaviour in two rat models of osteoarthritis. Ann. Rheum. Dis. 75(6), 1246-1254 (2016). https://doi.org/10. 1136/annrheumdis-2014-207203

210. M.S.M. Persson, J. Stocks, D.A. Walsh, M. Doherty, W. Zhang, The relative efficacy of topical non-steroidal antiinflammatory drugs and capsaicin in osteoarthritis: a network meta-analysis of randomised controlled trials. Osteoarthritis Cartil. 26(12), 1575-1582 (2018). https://doi.org/ 10.1016/j.joca.2018.08.008

211. P.I. Mapp, D.A. Walsh, Mechanisms and targets of angiogenesis and nerve growth in osteoarthritis. Nat. Rev. Rheumatol. 8(7), 390-398 (2012). https://doi.org/10.1038/nrrhe um.2012.80

212. S. Ashraf, D.A. Walsh, Angiogenesis in osteoarthritis. Curr. Opin. Rheumatol. 20(5), 573-580 (2008). https://doi.org/ 10.1097/BOR.0b013e3283103d12

213. M.P. Murphy, L.S. Koepke, M.T. Lopez, X. Tong, T.H. Ambrosi et al., Articular cartilage regeneration by activated skeletal stem cells. Nat. Med. 26(10), 1583-1592 (2020). https://doi.org/10.1038/s41591-020-1013-2

214. C.J. Avraamides, B. Garmy-Susini, J.A. Varner, Integrins in angiogenesis and lymphangiogenesis. Nat. Rev. Cancer 8(8), 604-617 (2008). https://doi.org/10.1038/nrc2353

215. P. Mukherjee, R. Bhattacharya, P. Wang, L. Wang, S. Basu et al., Antiangiogenic properties of gold nanoparticles. Clin. Cancer Res. 11(9), 3530-3534 (2005). https://doi. org/10.1158/1078-0432.Ccr-04-2482

216. T. Yang, Q. Yao, F. Cao, Q. Liu, B. Liu et al., Silver nanoparticles inhibit the function of hypoxia-inducible factor-1 and target genes: Insight into the cytotoxicity and antiangiogenesis. Int. J. Nanomed. 11, 6679-6692 (2016). https:// doi.org/10.2147/ijn.S109695

217. S. Gurunathan, K.J. Lee, K. Kalishwaralal, S. Sheikpranbabu, R. Vaidyanathan et al., Antiangiogenic properties 
of silver nanoparticles. Biomaterials 30(31), 6341-6350 (2009). https://doi.org/10.1016/j.biomaterials.2009.08.008

218. J. Kim, A.C. Mirando, A.S. Popel, J.J. Green, Gene delivery nanoparticles to modulate angiogenesis. Adv. Drug Deliv. Rev. 119, 20-43 (2017). https://doi.org/10.1016/j.addr. 2016.11 .003

219. M.A. Greene, R.F. Loeser, Aging-related inflammation in osteoarthritis. Osteoarthritis Cartil. 23(11), 1966-1971 (2015). https://doi.org/10.1016/j.joca.2015.01.008

220. J. Lieberthal, N. Sambamurthy, C.R. Scanzello, Inflammation in joint injury and post-traumatic osteoarthritis. Osteoarthritis Cartil. 23(11), 1825-1834 (2015). https://doi.org/ 10.1016/j.joca.2015.08.015

221. C.C. Wu, W.H. Chen, B. Zao, P.L. Lai, T.C. Lin et al., Regenerative potentials of platelet-rich plasma enhanced by collagen in retrieving pro-inflammatory cytokine-inhibited chondrogenesis. Biomaterials 32(25), 5847-5854 (2011). https://doi.org/10.1016/j.biomaterials.2011.05.002

222. C. Gao, Y. Wang, Z. Ye, Z. Lin, X. Ma et al., Biomedical micro-/nanomotors: from overcoming biological barriers to in vivo imaging. Adv. Mater. 33(6), e2000512 (2021). https://doi.org/10.1002/adma.202000512

223. P. Mi, Stimuli-responsive nanocarriers for drug delivery, tumor imaging, therapy and theranostics. Theranostics 10(10), 4557-4588 (2020). https://doi.org/10.7150/thno. 38069

224. S. Mura, J. Nicolas, P. Couvreur, Stimuli-responsive nanocarriers for drug delivery. Nat. Mater. 12(11), 991-1003 (2013). https://doi.org/10.1038/nmat3776

225. S. Jafari, L.O. Mair, S. Chowdhury, A. Nacev, R. Hilaman et al., Magnetically targeted delivery through cartilage. AIP Adv. 8(5), 056717 (2018). https://doi.org/10.1063/1.5006156

226. J.-Y. Su, S.-H. Chen, Y.-P. Chen, W.-C. Chen, Evaluation of magnetic nanoparticle-labeled chondrocytes cultivated on a type ii collagen-chitosan/poly(lactic-co-glycolic) acid biphasic scaffold. Int. J. Mol. Sci. 18(1), 87 (2017). https://doi.org/ 10.3390/ijms 18010087

227. B. Son, H.D. Kim, M. Kim, J.A. Kim, J. Lee et al., Physical stimuli-induced chondrogenic differentiation of mesenchymal stem cells using magnetic nanoparticles. Adv. Healthc. Mater. 4(9), 1339-1347 (2015). https://doi.org/10.1002/adhm.20140 0835

228. X.-Y. Zhou, X.-X. Zhang, G.-Y. Yu, Z.-C. Zhang, F. Wang et al., Effects of low-intensity pulsed ultrasound on knee osteoarthritis: A meta-analysis of randomized clinical trials. Biomed Res. Int. 2018, 7469197-7469197 (2018). https://doi. org/10.1155/2018/7469197

229. T. Nishida, S. Kubota, E. Aoyama, N. Yamanaka, K.M. Lyons et al., Low-intensity pulsed ultrasound (LIPUS) treatment of cultured chondrocytes stimulates production of ccn family protein 2 (CCN2), a protein involved in the regeneration of articular cartilage: Mechanism underlying this stimulation. Osteoarthritis Cartil. 25(5), 759-769 (2017). https://doi.org/ 10.1016/j.joca.2016.10.003

230. D. Gao, T. Chen, S. Chen, X. Ren, Y. Han et al., Targeting hypoxic tumors with hybrid nanobullets for oxygen-independent synergistic photothermal and thermodynamic therapy. Nano-Micro Lett. 13(1), 99 (2021). https:// doi.org/10.1007/s40820-021-00616-4

231. Z. Yang, D. Gao, X. Guo, L. Jin, J. Zheng et al., Fighting immune cold and reprogramming immunosuppressive tumor microenvironment with red blood cell membrane-camouflaged nanobullets. ACS Nano 14(12), 17442-17457 (2020). https://doi.org/10.1021/acsnano.0c07721

232. D. Gao, X. Guo, X. Zhang, S. Chen, Y. Wang et al., Multifunctional phototheranostic nanomedicine for cancer imaging and treatment. Mater. Today Bio 5, 100035 (2020). https:// doi.org/10.1016/j.mtbio.2019.100035

233. J. You, R. Zhang, C. Xiong, M. Zhong, M. Melancon et al., Effective photothermal chemotherapy using doxorubicinloaded gold nanospheres that target EPHB4 receptors in tumors. Cancer Res. 72(18), 4777-4786 (2012). https://doi. org/10.1158/0008-5472.Can-12-1003

234. A.W. Visser, R. de Mutsert, S. le Cessie, M. den Heijer, F.R. Rosendaal et al., The relative contribution of mechanical stress and systemic processes in different types of osteoarthritis: the neo study. Ann. Rheum. Dis. 74(10), 1842-1847 (2015). https://doi.org/10.1136/annrheumdis-2013-205012

235. M.N. Holme, I.A. Fedotenko, D. Abegg, J. Althaus, L. Babel et al., Shear-stress sensitive lenticular vesicles for targeted drug delivery. Nat. Nanotechnol. 7(8), 536-543 (2012). https://doi.org/10.1038/nnano.2012.84

236. R. Rajamanickam, S. Baek, K. Gwon, Y. Hwang, K. Shin et al., Mechanical stimuli responsive and highly elastic biopolymer/nanoparticle hybrid microcapsules for controlled release. J. Mater. Chem. B 4(24), 4278-4286 (2016). https://doi.org/10.1039/c6tb00410e

237. M. Deloney, K. Smart, B.A. Christiansen, A. Panitch, Thermoresponsive, hollow, degradable core-shell nanoparticles for intra-articular delivery of anti-inflammatory peptide. J. Control. Release 323, 47-58 (2020). https://doi.org/10. 1016/j.jconrel.2020.04.007

238. Y. Wang, D. Gao, Y. Liu, X. Guo, S. Chen et al., Immunogenic-cell-killing and immunosuppression-inhibiting nanomedicine. Bioact. Mater. 6(6), 1513-1527 (2021). https:// doi.org/10.1016/j.bioactmat.2020.11.016

239. S.M. Horvath, J.L. Hollander, Intra-articular temperature as a measure of joint reaction. J. Clin. Invest. 28(3), 469-473 (1949). https://doi.org/10.1172/jci102092

240. N.A. Cummings, G.L. Nordby, Measurement of synovial fluid $\mathrm{pH}$ in normal and arthritic knees. Arthritis Rheum. 9(1), 47-56 (1966). https://doi.org/10.1002/art.1780090106

241. S. Khanal, U. Adhikari, N.P. Rijal, S.R. Bhattarai, J. Sankar et al., $\mathrm{pH}$-responsive plga nanoparticle for controlled payload delivery of diclofenac sodium. J. Funct. Biol. 7(3), 21 (2016). https://doi.org/10.3390/jfb7030021

242. M.F. Chung, W.T. Chia, W.L. Wan, Y.J. Lin, H.W. Sung, Controlled release of an anti-inflammatory drug using an ultrasensitive ros-responsive gas-generating carrier for localized inflammation inhibition. J. Am. Chem. Soc. 137(39), 12462-12465 (2015). https://doi.org/10.1021/jacs. 5 b08057 
243. D. Olszewska-Slonina, S. Jung, D. Matewski, K.J. Olszewski, E. Krzyzynska-Malinowska et al., Lysosomal enzymes in serum and synovial fluid in patients with osteoarthritis. Scand. J. Clin. Lab. Invest. 75(2), 145-151 (2015). https:// doi.org/10.3109/00365513.2014.992946

244. Z. Jia, L. Song, F. Zang, J. Song, W. Zhang et al., Active-target $\mathrm{t} 1$-weighted MR imaging of tiny hepatic tumor via RGD modified ultra-small $\mathrm{Fe}_{3} \mathrm{O}_{4}$ nanoprobes. Theranostics 6(11), 1780-1791 (2016). https://doi.org/10.7150/thno.14280

245. Y.M. Soshnikova, S.G. Roman, N.A. Chebotareva, O.I. Baum, M.V. Obrezkova et al., Starch-modified magnetite nanoparticles for impregnation into cartilage. J. Nanopart. Res. 15(11), 2092 (2013). https://doi.org/10.1007/s11051-013-2092-5

246. C. Bai, Z. Jia, L. Song, W. Zhang, Y. Chen et al., Timedependent $\mathrm{t} 1 \mathrm{-} \mathrm{t} 2$ switchable magnetic resonance imaging realized by c (RGDYK) modified ultrasmall $\mathrm{Fe}_{3} \mathrm{O}_{4}$ nanoprobes. Adv. Funct. Mater. 28(32), 1802281 (2018). https://doi.org/ 10.1002/adfm.201802281

247. A.J. Mieszawska, W.J.M. Mulder, Z.A. Fayad, D.P. Cormode, Multifunctional gold nanoparticles for diagnosis and therapy of disease. Mol. Pharm. 10(3), 831-847 (2013). https://doi. org/10.1021/mp3005885

248. S. Peng, Q. Zheng, X. Zhang, L. Dai, J. Zhu et al., Detection of adamts-4 activity using a fluorogenic peptide-conjugated au nanoparticle probe in human knee synovial fluid. ACS Appl. Mater. Interfaces 5(13), 6089-6096 (2013). https://doi. org/10.1021/am400854z

249. V.C. Pierre, M. Botta, S. Aime, K.N. Raymond, Substituent effects on Gd(iii)-based MRI contrast agents: optimizing the stability and selectivity of the complex and the number of coordinated water molecules. Inorg. Chem. 45(20), 83558364 (2006). https://doi.org/10.1021/ic061262q

250. T. Kim, E. Momin, J. Choi, K. Yuan, H. Zaidi et al., Mesoporous silica-coated hollow manganese oxide nanoparticles as positive $\mathrm{t} 1$ contrast agents for labeling and mri tracking of adipose-derived mesenchymal stem cells. J. Am. Chem. Soc. 133(9), 2955-2961 (2011). https://doi.org/10. 1021/ja1084095

251. P.H. Kuo, E. Kanal, A.K. Abu-Alfa, S.E. Cowper, Gadolinium-based MR contrast agents and nephrogenic systemic fibrosis. Radiology 242(3), 647-649 (2007). https://doi.org/ 10.1148/radiol.2423061640

252. J. Chen, J. Qi, C. Chen, J. Chen, L. Liu et al., Tocilizumabconjugated polymer nanoparticles for NIR-ii photoacousticimaging-guided therapy of rheumatoid arthritis. Adv. Mater. (2020). https://doi.org/10.1002/adma.202003399

253. J. Ouyang, X. Ji, X. Zhang, C. Feng, Z. Tang et al., In situ sprayed NIR-responsive, analgesic black phosphorus-based gel for diabetic ulcer treatment. Proc. Natl. Acad. Sci. U. S. A. 117(46), 28667-28677 (2020). https://doi.org/10.1073/ pnas. 2016268117

254. L. Jin, X. Guo, D. Gao, C. Wu, B. Hu et al., Nir-responsive mxene nanobelts for wound healing. NPG Asia Mater. 13(1), 24 (2021). https://doi.org/10.1038/s41427-021-00289-w

255. P. Jin, C. Wiraja, J. Zhao, J. Zhang, L. Zheng et al., Nitric oxide nanosensors for predicting the development of osteoarthritis in rat model. ACS Appl. Mater. Interfaces 9(30), 25128-25137 (2017). https://doi.org/10.1021/acsami. $7 \mathrm{~b} 06404$

256. L. Zhou, V.O. Gjvm, J. Malda, M.J. Stoddart, Y. Lai et al., Innovative tissue-engineered strategies for osteochondral defect repair and regeneration: Current progress and challenges. Adv. Healthc. Mater. (2020). https://doi.org/10.1002/ adhm. 202001008

257. S.T. Grady, L. Britton, K. Hinrichs, A.J. Nixon, A.E. Watts, Persistence of fluorescent nanoparticle-labelled bone marrow mesenchymal stem cells in vitro and after intra-articular injection. J. Tissue Eng. Regen. Med. 13(2), 191-202 (2019). https://doi.org/10.1002/term.2781

258. J.M. Lee, B.S. Kim, H. Lee, G.I. Im, In vivo tracking of mesechymal stem cells using fluorescent nanoparticles in an osteochondral repair model. Mol. Ther. 20(7), 1434-1442 (2012). https://doi.org/10.1038/mt.2012.60

259. H. Markides, O. Kehoe, R.H. Morris, A.J. El. Haj, Whole body tracking of superparamagnetic iron oxide nanoparticlelabelled cells-a rheumatoid arthritis mouse model. Stem Cell Res. Ther. 4(5), 126-126 (2013). https://doi.org/10.1186/ scrt337

260. J. Chen, F. Wang, Y. Zhang, X. Jin, L. Zhang et al., In vivo tracking of superparamagnetic iron oxide nanoparticle labeled chondrocytes in large animal model. Ann. Biomed. Eng. 40(12), 2568-2578 (2012). https://doi.org/10.1007/ s10439-012-0621-5

261. G. Parekh, Y. Shi, J. Zheng, X. Zhang, S. Leporatti, Nanocarriers for targeted delivery and biomedical imaging enhancement. Ther. Deliv. 9(6), 451-468 (2018). https:// doi.org/10.4155/tde-2018-0013

262. P. Singh, S. Pandit, V. Mokkapati, A. Garg, V. Ravikumar et al., Gold nanoparticles in diagnostics and therapeutics for human cancer. Int. J. Mol. Sci. (2018). https://doi.org/ 10.3390/ijms 19071979

263. M. Moglianetti, E. De. Luca, D. Pedone, R. Marotta, T. Catelani et al., Platinum nanozymes recover cellular ros homeostasis in an oxidative stress-mediated disease model. Nanoscale 8(6), 3739-3752 (2016). https://doi.org/10.1039/ C5NR08358C

264. Y. Vlamidis, V. Voliani, Bringing again noble metal nanoparticles to the forefront of cancer therapy. Front. Bioeng. Biotechnol. 6, 143-143 (2018). https://doi.org/10.3389/ fbioe.2018.00143

265. K. Memarzadeh, A.S. Sharili, J. Huang, S.C. Rawlinson, R.P. Allaker, Nanoparticulate zinc oxide as a coating material for orthopedic and dental implants. J. Biomed. Mater. Res. Part A 103(3), 981-989 (2015). https://doi.org/10. 1002/jbm.a.35241

266. J.-L. Wang, J.-K. Xu, C. Hopkins, D.H.-K. Chow, L. Qin, Biodegradable magnesium-based implants in orthopedics-a general review and perspectives. Adv. Sci. 7(8), 19024431902443 (2020). https://doi.org/10.1002/advs.201902443

267. J.H. Kim, J. Jeon, M. Shin, Y. Won, M. Lee et al., Regulation of the catabolic cascade in osteoarthritis by the 
zinc-ZIP8-MTF1 axis. Cell 156(4), 730-743 (2014). https://doi.org/10.1016/j.cell.2014.01.007

268. H. Yao, J.K. Xu, N.Y. Zheng, J.L. Wang et al., Intra-articular injection of magnesium chloride attenuates osteoarthritis progression in rats. Osteoarthr. Cartil. 27(12), 18111821 (2019). https://doi.org/10.1016/j.joca.2019.08.007

269. W.L. Wan, Y.J. Lin, P.C. Shih, Y.R. Bow, Q. Cui et al., An in situ depot for continuous evolution of gaseous $\mathrm{H}_{2}$ mediated by a magnesium passivation/activation cycle for treating osteoarthritis. Angew. Chem. Int. Ed. 57(31), 9875-9879 (2018). https://doi.org/10.1002/anie.201806159

270. Z. Tang, N. Kong, X. Zhang, Y. Liu, P. Hu et al., A materials-science perspective on tackling covid-19. Nat. Rev. Mater. (2020). https://doi.org/10.1038/s41578-020-00247-y

271. Z. Lei, W. Zhu, X. Zhang, X. Wang, P. Wu, Bio-inspired ionic skin for theranostics. Adv. Funct. Mater. 31(8), 2008020 (2021). https://doi.org/10.1002/adfm.202008020

272. Z. Li, D. Chu, Y. Gao, L. Jin, X. Zhang et al., Biomimicry, biomineralization, and bioregeneration of bone using advanced three-dimensional fibrous hydroxyapatite scaffold. Mater. Today Adv. 3, 100014 (2019). https://doi.org/ 10.1016/j.mtadv.2019.100014
273. C.L. Ventola, Progress in nanomedicine: approved and investigational nanodrugs. Pharm. Ther. 42(12), 742-755 (2017)

274. W. Xinluan, L. Yuxiao, N.H. Helena, Y. Zhijun, Q. Ling, Systemic drug delivery systems for bone tissue regeneration-a mini review. Curr. Pharm. Des. 21(12), 1575-1583 (2015). https://doi.org/10.2174/1381612821666150115152841

275. N. Bodick, J. Lufkin, C. Willwerth, A. Kumar, J. Bolognese et al., An intra-articular, extended-release formulation of triamcinolone acetonide prolongs and amplifies analgesic effect in patients with osteoarthritis of the knee: a randomized clinical trial. J. Bone Jt. Surg. Am. 97(11), 877-888 (2015). https://doi.org/10.2106/jbjs.N.00918

276. S.G. Owen, H.W. Francis, M.S. Roberts, Disappearance kinetics of solutes from synovial fluid after intra-articular injection. Br. J. Clin. Pharmacol. 38(4), 349-355 (1994). https://doi.org/10.1111/j.1365-2125.1994.tb04365.x

277. M.B. Goldring, J.-M. Dayer, S.R. Goldring, in Chapter 14Osteoarthritis and the Immune System, eds. by J. Lorenzo, M.C. Horowitz, Y. Choi et al. (Academic Press, San Diego, 2016), pp. 257-269 